\title{
Analysis of the mechanisms of interaction of alpha-Synuclein and membranes in cellular models of Parkinson's Disease.
}

\author{
Dissertation \\ For the award of the Degree \\ "Doctor of Philosophy" \\ Division of Biology
}

Of the Georg-August University Göttingen

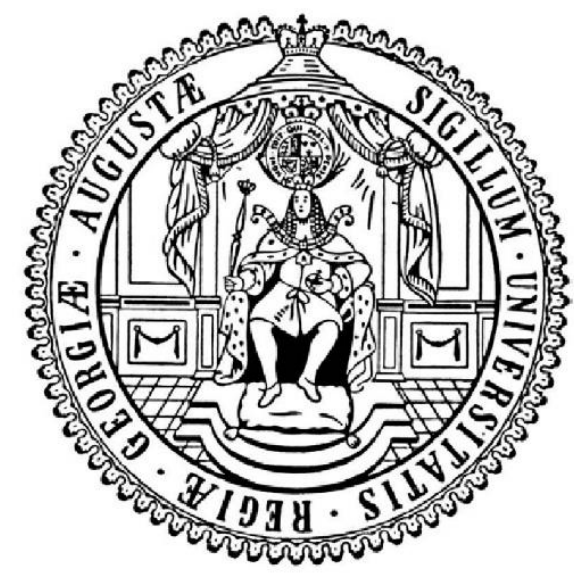

Within the Doctoral program of the

Georg-August University School of Science (GAUSS)

and

Centro Universitario Argentino Alemán (CUAA - DAHZ)

International PhD Program in Molecular Biosciences and Biomedicine

Submitted by: Caterina Masaracchia

From: Salemi (TP) - Italy -

Göttingen, 2018 



\section{Thesis Committee:}

- Prof. Dr. Tiago F. Outeiro (Supervisor)

Department of Experimental Neurodegeneration,

University Medical Center (UMG) Göttingen

- Prof. Dr. Markus Zweckstetter

Protein structure determination using NMR,

German Center for Neurodegenerative Diseases (DZNE) Göttingen

- Prof. Dr. Gerhard Braus

Department of Microbiology and Genetics, Georg August University Göttingen

\section{Members of the Examination Board:}

1st Referee: Prof. Dr. Tiago F. Outeiro

Department of Experimental Neurodegeneration,

University Medical Center (UMG) Göttingen

2nd Referee: Prof. Dr. Markus Zweckstetter

Protein structure determination using NMR,

German Center for Neurodegenerative Diseases (DZNE) Göttingen

\section{Further members of the Examination Board:}

- Prof Dr. Claudio O. Fernández (Co-Supervisor) (External $3^{\text {rd }}$ Referee) Institute for the Drug discovery of Rosario (IIDEFAR, CONICET-UNR)

Max Planck Laboratory for Structural Biology, Chemistry and Molecular Biophysics of Rosario (MPLbioR, UNR-Sociedad Max Planck) - Rosario, Santa Fe - Argentina

- Prof. Dr. Reinhard Jahn

Department of Neurobiology

Max Planck Institute for Biophysical Chemistry Göttingen

- Dr. Ira Milosevic

Synaptic Vesicle Dynamics, European Neuroscience Institute (ENI) Göttingen

- Prof. Dr. Mauricio Menacho Marquez

Institute for the Drug discovery of Rosario (IIDEFAR, CONICET-UNR)

Max Planck Laboratory for Structural Biology, Chemistry and Molecular Biophysics

of Rosario (MPLbioR, UNR-Sociedad Max Planck) - Rosario, Santa Fe - Argentina

Date of oral examination: Tuesday, $17^{\text {th }}$ of April, 2018 



\section{AFFIDAVIT}

I hereby declare that the doctoral thesis entitled "Analysis of the mechanisms of interaction of alpha-Synuclein and membranes in cellular models of Parkinson's Disease" has been written independently, with no other sources and aids than quoted.

Caterina Masaracchia,

Göttingen, March 2018 

Dedico questo lavoro principalmente alla mia incredibile Mamma, Francesca, Donna fortissima.

Ed al mio Papino Bellissimo, Gaspare, che mi ha insegnato ad essere un uomo. 



\section{TABLE OF CONTENTS}

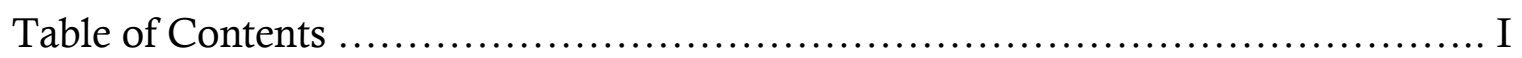

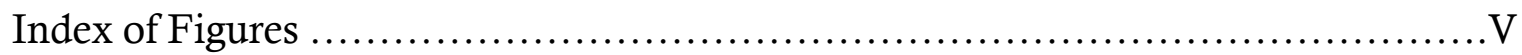

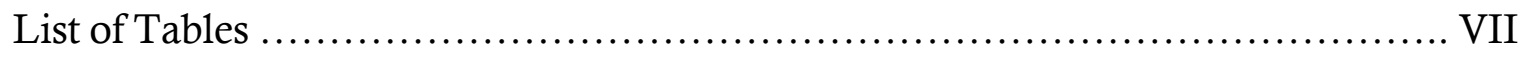

List of Abbreviations ...............................................................

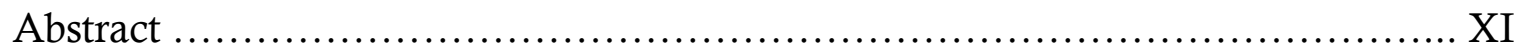

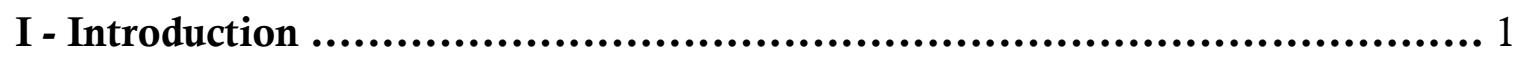

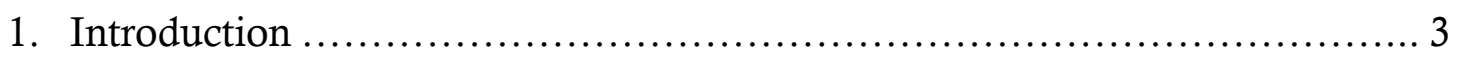

1.1 Alpha-Synuclein and Synucleinopathies .................................... 3

1.1.1 Life expectancy, aging and neurodegenerative disorders ..................... 3

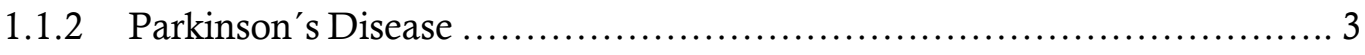

1.1.3 Proteinopathies and synucleinopathies ....................................... 5

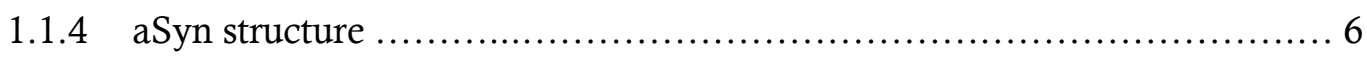

1.2 aSyn: physiological function and pathological behaviour .................... 9

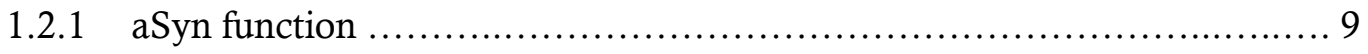

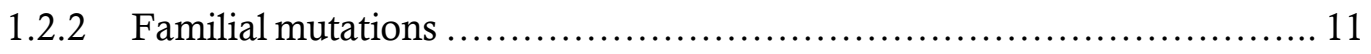

1.2.3 Post-translational modifications ........................................... 12

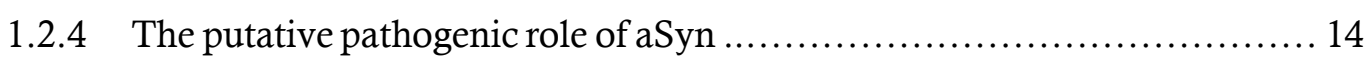

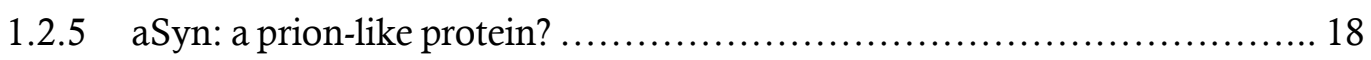

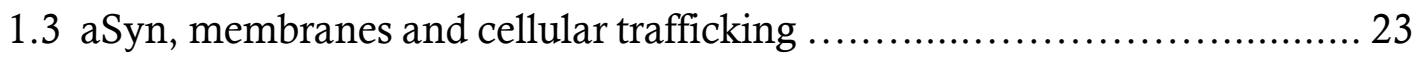

1.3.1 aSyn and membranes ….................................................. 23

1.3.2 aSyn and the RAB-GTPases family proteins ............................ 25

1.3.3 Uptake, processing and clearance of aSyn ............................... 32

1.4 Cellular models for the study of aSyn pathology ............................ 36

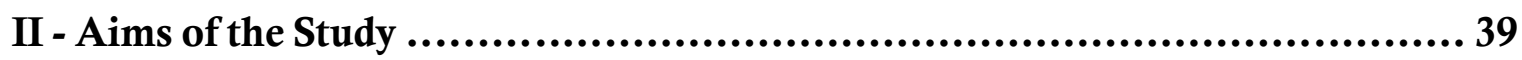


III - Materials and Methods .................................................. 43

3. Materials and Methods ................................................. 45

3.1 Purification and characterization of recombinant proteins .............. 45

3.1.1 Protein purification .................................................... 45

3.1.2 Fibril formation ....................................................... 46

3.1.3 Thioflavine T assay ..................................................... 46

3.1.4 Transmission Electron Microscopy ................................... 47

3.2 Cell culture and molecular biology techniques ....................... 47

3.2.1 Human cell culture .................................................... 47

3.2.2 Transient transfection of cultured cells ................................ 48

3.2.3 Solubilisation of proteins and protein quantification ...................... 48

3.2.4 Western Blot Analyses ..................................................... 49

3.2.5 Dot Blot Analyses .................................................... 49

3.2.6 Triton X-100 fractionation assay ........................................ 50

3.2.7 Biotinylation assay ............................................... 50

3.3 Immunofluorescence, microscopy and imaging .................... 52

3.3.1 Immunocytochemistry ................................................ 52

3.3.2 Microscopy and imaging ........................................... 53

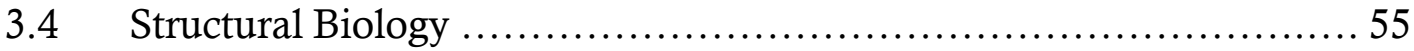

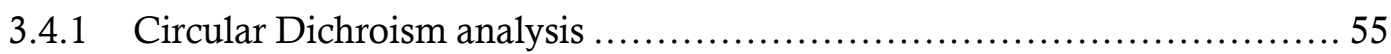

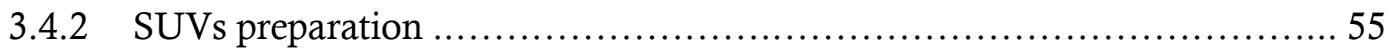

3.4.3 Nuclear Magnetic Resonance ............................................ 55

3.5 Software and statistics ............................................ 57

IV - Results ...................................................................... 59

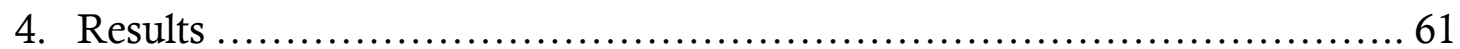

4.1 Uptake of recombinant aSyn species in cultured cells .................... 61

4.1.1 aSyn enters $\mathrm{H} 4$ cells and forms intracellular inclusions .................... 61 
4.1.2 aSyn interacts with plasma membrane and forms high molecular weight species

4.2 RAB-GTPase proteins modulate aSyn internalization and aggregation ..... 66

4.2.1 aSyn partially colocalizes with Rab 5A and Rab 7 ........................ 68

4.2.2 aSyn forms inclusions surrounded by Rab 4A positive vesicles .............. 70

4.3 aSyn membrane binding properties .................................. 71

4.3.1 Membrane binding properties are essential for the internalization and the inclusions formation of aSyn in $\mathrm{H} 4$ cells .................................... 71

4.3.2 aSyn A11P/V70P is unable to bind membranes .......................... 74

4.3.3 aSyn A30P and aSyn A11P/V70P are less internalized in Rab 4A and Rab $5 \mathrm{~A}$ transfected cells 77

4.3.4 Overexpression of $\mathrm{Rab} 7$ reduces the formation of dimers in cells treated with aSyn WT

4.4 The endocytic pathway and the ALP are involved in the processing of aSyn 81

4.4.1 The internalization of aSyn is mediated by dynamin ...................... 81

4.4.2 Impairment of autophagy inhibits the degradation of aSyn ............... 84

4.5 Biophysical characterization of SynT …............................. 87

4.5.1 C-terminally modified aSyn forms inclusions in $\mathrm{H} 4$ cells .................. 87

4.5.2 The intrinsically disordered nature of aSyn is not influenced by the Cterminal modification in SynT .......................................... 89

4.5.3 Transient interactions between the $\mathrm{N}$ - and the C-terminal regions are strengthened in SynT ................................................. 92

4.5.4 The N-terminal region of SynT is less available than in aSyn ............. 93

4.5.5 SynT does not form amyloid fibrils in vitro ............................... 94

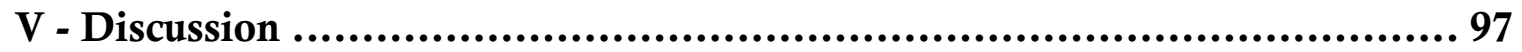

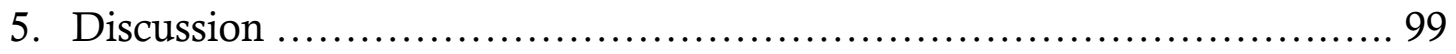

5.1 aSyn membrane binding properties are essential for its internalization .. 100

5.2 The RABs screening and Rab 4A: an important player in aSyn pathology? 102 
5.3 aSyn is internalized via a dynamin-dependent mechanism 104

5.4 Extracellular monomeric aSyn might be sufficient to initiate the spreading

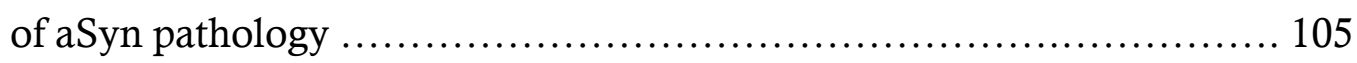

5.5 C-terminal modification in SynT and its effect on aggregation propensity

VI - Conclusions

6. Conclusion 115

VII - Bibliography

7. Bibliography. 119

Acknowledgements 137

Curriculum Vitae. 141 


\section{INDEX OF FIGURES}

Figure 1. Pathological hallmark of Parkinson's Disease ................................ 4

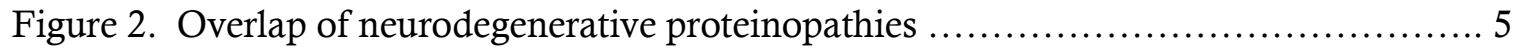

Figure 3. Schematic representation of the domains and structure of aSyn .................. 8

Figure 4. Schematic representation of aSyn familial mutation, PTMs and map of the interactor protein binding site ............................................ 13

Figure 5. Mechanisms of aSyn aggregation ............................................ 16

Figure 6. aSyn physiological function and pathological behaviour ....................... 17

Figure 7. Braak's staging hypothesis for the progression of PD pathology ................. 18

Figure 8. Potential mechanisms mediating cell-to-cell transmission of aSyn ................ 20

Figure 9. Proposed model for aSyn folding and conformational switching $\ldots \ldots \ldots \ldots \ldots \ldots . \ldots 23$

Figure 10. Schematic illustration of the different roles of the three regions of aSyn and their interaction with lipid bilayers ................................................. 25

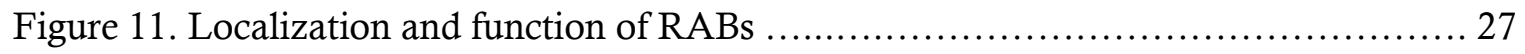

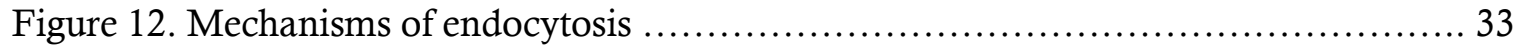

Figure 13. Main type of autophagy in mammalian cells .................................. 35

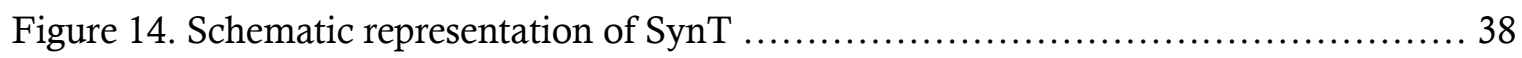

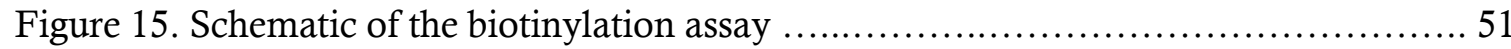

Figure 16. Recombinant aSyn monomers and fibrils are internalized by H4 cells ............ 62

Figure 17. aSyn associates with membranes during the internalization process in H4 cells . 64

Figure 18. aSyn forms high molecular weight species in $\mathrm{H} 4$ cells ........................... 65

Figure 19. aSyn partially colocalizes with Rab 5A-GFP and Rab 7-GFP in H4 cells .......... 69

Figure 20. aSyn forms inclusions surrounded by Rab 4A positive vesicles in $\mathrm{H} 4$ cells ........ 71 
Figure 21. Membrane binding is essential for the internalization and inclusions formation of aSyn in $\mathrm{H} 4$ cells 73

Figure 22. Characterization of aSyn A11P/V70P mutant 76

Figure 23. aSyn A30P and A11P/V70P are less internalized in Rab 4A-GFP, Rab 5A-GFP and Rab 5ACA-GFP transfected cells compared to aSyn WT 78

Figure 24. Rab 7 decreases the formation of dimers in H4 cells treated with aSyn WT monomers 80

Figure 25. Dyngo effectively prevents the internalization of aSyn in H4 cells overexpressing $\mathrm{Rab} 4 \mathrm{~A}$, whereas PitStop does not have a significant effect 82

Figure 26. Dyngo effectively prevents the internalization of aSyn in $\mathrm{H} 4$ cells, whereas PitStop has the opposite effect 83

Figure 27. Impairment of autophagy inhibits the degradation of aSyn in H4 cells transfected with Rab 7-GFP 85

Figure 28. Impairment of autophagy inhibits the degradation of aSyn in $\mathrm{H} 4$ cells 86

Figure 29. C-terminal modified aSyn (SynT) forms inclusions in H4 cells 88

Figure 30. Biophysical characterization of SynT 90

Figure 31. NMR characterization of SynT 91

Figure 32. Backbone dynamics of SynT 95

Figure 33. Binding and aggregation properties of SynT 96

Figure 34. RABs membranes domains 103

Figure 35. Proposed mechanism of aSyn internalization and interaction with the membrane trafficking machinery pathway 108

Figure 36. Proposed model for the structural properties of SynT 111 


\section{LIST OF TABLES}

Table A. The RABs family: intracellular location, known pathways, effectors and related diseases 31

Table B. Primary and Secondary Antibodies 55

Table C. Selected RABs from the screen 67 


\section{LIST OF ABBREVIATIONS}

aa - amino acid

$\mathrm{AD}$ - Alzheimer's Disease

AEC - Anion Exchange Chromatography

ALP - Autophagy/Lysosomal Pathway

$A \beta$ - amyloid-beta

APS - Ammonium Persulfate

aSyn - alpha-synuclein

aSyn WT - aSyn Wild-Type

BiFC - Bimolecular Fluorescence Complementation assay

BSA - Bovine Serum Albumin

CaPho - Calcium Phosphate

CCVs - Clathrin Coated Vesicles

CD - Circular Dichroism

CMA - Chaperone Mediated Autophagy

CME - Clathrin Mediated Endocytosis

CNS - Central Nervous System

CSF - Cerebrospinal Fluid

Cys - cysteine

DLB - Dementia with Lewy Bodies

EGFP - Enhanced Green Fluorescent Protein

EPR - Electron Paramagnetic Resonance

ER - Endoplasmic Reticulum

FBS - Foetal Bovine Serum

GAPs - GTPase-Activating Proteins

GDF - GDI displacement factor 
GDI - GDP Dissociation Inhibitor

GEFs - Guanine nucleotide Exchange Factors

hetNOEs - Steady-state ${ }^{1} \mathrm{H}_{-}{ }^{15} \mathrm{~N}$ NOE

ICC - Immunocytochemistry

IDPs - Intrinsically Disordered Proteins

IPTG - isopropyl-1-thio- $\beta$-D-galactopyranoside

${ }^{3} \mathrm{~J} \mathrm{HN}-\mathrm{H} \alpha$ - Three-bond HN-H $\alpha$ coupling constants

LAMP2A - Lysosome Associated Membrane Protein type 2A

LBs - Lewy Bodies

LNs - Lewy Neurites

Lys - Lysine

MSA - Multiple System Atrophy

NMR - Nuclear Magnetic Resonance

NAC - Non-Amyloid Component

NatB - N-terminal acetyltransferase B complex

PBS - phosphate saline buffer

PD - Parkinson's Disease

PFA - Paraformaldehyde

Pro - proline

PTMs - Post Translational Modification

RABs - Rab GTPases family proteins

RIPA Buffer - radioimmuniprecipitation assay buffer

ROS - Reactive Oxygen Species

RT - Room Temperature

SDS - Sodium Dodecyl Sulphate

SDS-PAGE - Sodium Dodecyl Sulphate Polyacrylamide Gel Electrophoresis

SEC - Size Exclusion Chromatography

Ser/Thr - Serine/Threonine 
SNpc - Substantia Nigra pars compacta

SUVs - Small Unilamellar Vesicles

TBS - Tris buffered saline

TBS-T - Tris buffered saline supplemented with Tween-20

TEMED - tetramethylethylenediamine

TRIS - Tris (hydroxymethyl)-aminomethane

ThioS - Thioflavine S

ThioT - Thioflavine T

TGN - Trans-Golgi Network

Tyr - Tyrosine

UPS - Ubiquitine Proteasome System

WT - Wild-Type 


\section{ABSTRACT}

Alpha-synuclein (aSyn) plays a crucial role in Parkinson's disease (PD) and other synucleinopathies, since it misfolds and accumulates in typical proteinaceous inclusions known as Lewy bodies. The physiological function of aSyn is thought to be related to vesicle binding and trafficking, but the precise molecular mechanisms leading to aSyn pathogenicity are still obscure.

Based on studies using patient-derived brain tissue, animal models, and in vitro experiments, it has been hypothesized that aSyn pathology can spread in a prion-like manner and be transferred between interconnected neurons, contributing to the propagation of the disease. This hypothesis assumes that pathogenic aSyn may act as template to seed the aggregation of non-pathogenic protein.

In the present study we investigated the molecular mechanisms involved in the interaction of aSyn with membranes and the trafficking machinery in cellular models of PD. First, we demonstrated that different species of aSyn can enter cells and form high molecular weight species. By screening a pool of small GTPases family proteins, the RABs, we found that aSyn partially colocalizes with Rab 5A and Rab 7, suggesting the involvement of the endocytic pathway and of the autophagy-lysosomal pathway in the internalization and processing of aSyn monomers. Additionally, Rab $4 \mathrm{~A}$ seems to play an important and active role in the internalization of aSyn, although further data are needed in order to clarify the molecular mechanism and the effectors involved.

We also demonstrated that membrane binding is essential for the internalization of aSyn and the consequent interaction with the selected pathways. Taken together, our results suggest that the uptake of aSyn monomers might be sufficient to initiate the spreading of aSyn pathology.

We also investigated the structural features of a variant of aSyn, known as SynT, which renders aSyn more prone to aggregation in cell models. Using Nuclear Magnetic Resonance (NMR) we performed a detailed structural characterization of SynT through a systematic comparison with the unmodified aSyn. We found that the conformations adopted by SynT resemble those described for the unmodified protein. However, subtle differences were observed at the $\mathrm{N}$-terminal region involving transient intra and/or intermolecular interactions. Our results indicate that disturbances in the $\mathrm{N}$-terminal region of SynT, and the consequent decrease in membrane binding of the modified protein, might contribute to the pathobiology of aSyn. 
This work emphasizes the importance of membrane binding properties in the physiological and pathological function of aSyn, and the fundamental role of RAB proteins in the modulation of aSyn processing, clearance and spreading.

Taken together, our results suggest that targeting the activity of RAB proteins may hold important therapeutic value in $\mathrm{PD}$. 


$$
\begin{gathered}
-I- \\
\text { INTRODUCTION }
\end{gathered}
$$




\subsection{ALPHA-SYNUCLEIN AND SYNUCLEINOPATHIES}

\subsubsection{Life Expectancy, Aging And Neurodegenerative DISORDERS}

In the $20^{\text {th }}$ century, the tremendous increase of life expectancy has been one of the biggest accomplishments and definitely, a major goal of human race since its existence.

In high-income and in developing countries, national life expectancy has increased steadily for decades and projection studies suggest we will break the 90 -year barrier by $2030^{1-3}$. This development has been achieved due to medical progresses, advances in public health, growing life standards and a consequential drop in child mortality ${ }^{1-3}$.

Despite increasing of life expectancy do reflect a positive development, new challenges arises: the aging process is still intrinsically correlated with a biological and cognitive decline.

Age is a major, and perhaps most important, risk factor for cardiovascular diseases and several types of cancer. The risk to develop neurodegenerative disorders also increases significantly in the elderly population, representing the largest cause of disability in the world ${ }^{4}$.

Because of these serious, negative effects, aging is considered one of the greatest social and economic challenges that the world will face in the upcoming years ${ }^{5}$. Chronic neurodegenerative disorders and the related mental health deterioration (dementia) are often mentioned as especially problematic, imposing a significant burden on working relatives and requiring substantial expenses for professional care ${ }^{5}$.

\subsubsection{PARKINSON'S DISEASE}

Among the neurodegenerative disorders, Parkinson's disease (PD) is recognized as the second most common after Alzheimer's Disease (AD), and as the most diffuse movement disorder, affecting $1 \%$ of the population above 65 years old ${ }^{6}$.

PD was first described as the "shaking palsy" in 1817 by James Parkinson and the classical clinical manifestations of the disease include motor symptoms as bradykinesia, rigidity, resting tremor and postural instability. Non-motor symptoms include depression, anxiety 
and irritability, cognitive and personality changes, hallucinations, sleep disorders and hyposmia ${ }^{7-10}$.

Pathologically, the primary brain region affected in $\mathrm{PD}$ is the substantia nigra pars compacta $(\mathrm{SNpc})$ that consists of pigmented, dopaminergic neurons that project into the putamen. The peculiar degeneration of this specific neurons and the consequent development of dystrophic striatal projections are hallmarks of $\mathrm{PD}^{7,11}$ (Figure 1A).

Another pathological hallmark of $\mathrm{PD}$ is the presence in the neuronal body of intracellular proteinaceous inclusions commonly known as Lewy bodies (LB) and Lewy neurites (LN). These inclusions are characterized by the presence of the protein alpha-synuclein (aSyn) $)^{7,12,13}$ (Figure 1B).

$S N C A$, the gene that encodes for aSyn, was the first gene associated with autosomaldominant forms of $\mathrm{PD}^{14}$. Although mutations in $S N C A$ are overall rare, several point mutations, together with duplications and triplications of the $S N C A$ gene have been reported. Misfolded or excess aSyn proteins may cluster together to form LB and impair the function of these neurons in specific regions of the brain.

Thus, over the last two decades, the study of aSyn has been a major, fundamental goal for the scientific community, as it was hoped it could shed light into the molecular mechanisms associated with PD, thereby leading to novel, effective therapeutic strategies against PD and other neurodegenerative disorders.
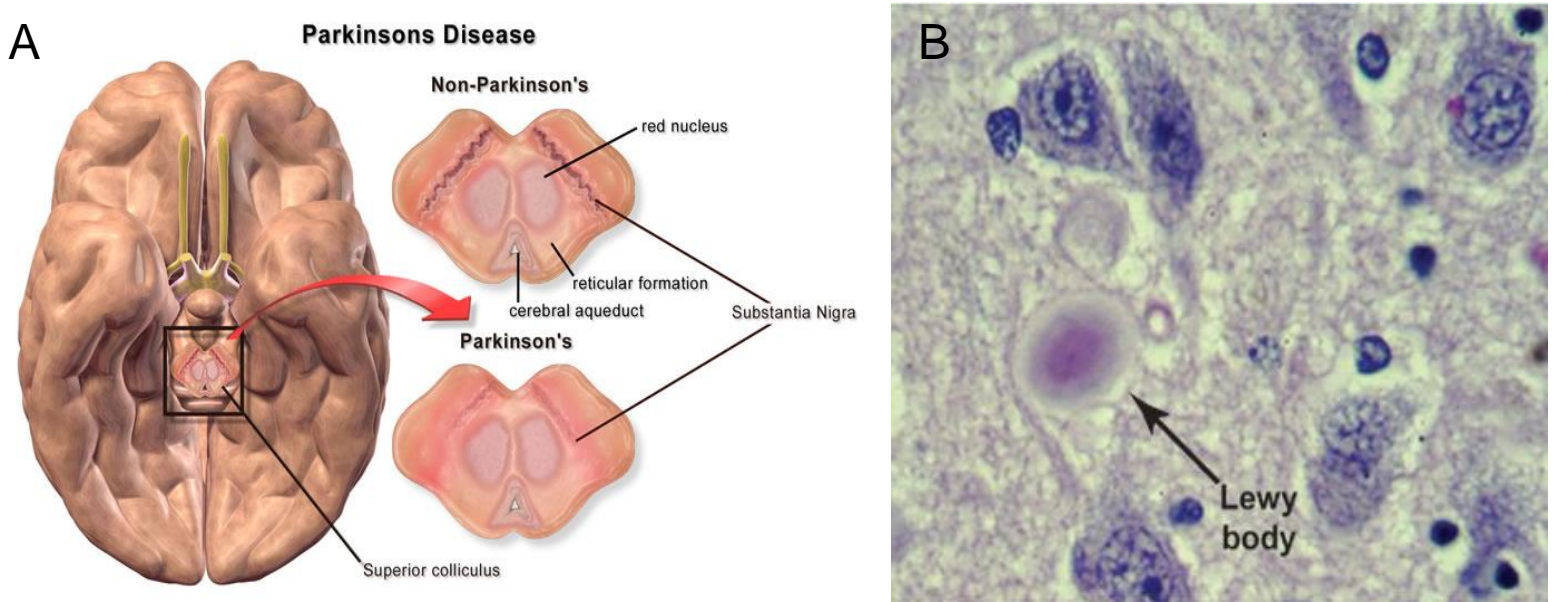

Figure 1. Pathological hallmark of Parkinson's Disease

(A) Representation of the differences between the SNpc region of a healthy brain and a brain with Parkinson's Disease. It is possible to see the lack of pigmented, dopaminergic neurons in the region of interest (Image credit: Blausen Gallery 2014 / Wikiversity Journal of Medicine, doi: 10.15347/wjm/2014.010). (B) ICC of a neuronal population from PD brain. The presence of Lewy body and its characteristic halo-like morphology is highlighted. (From National Library of Medicine, NIH) 


\subsubsection{PRoteInOPATHIES AND SyNUCLEINOPATHIES}

The aggregation and accumulation of proteins inside or outside cells in the brain is a common feature among many neurodegenerative disorders such as AD, PD, Huntington's disease, or spongiform encephalopathies ${ }^{15}$. Consequently, these diseases are also referred to as proteinopathies.

The general principle of proteinopathies is that the proteins involved change their conformation, either gaining toxic activity or losing their normal functions.

In the healthy brain, these proteins are found in a monomeric, physiological form.

In disease, proteins experience conformational changes leading to the formation of abnormal inclusions that accumulate in aggregates or in higher order structures ${ }^{16}$.

Different diseases are primarily associated with the accumulation of particular types of protein aggregates. However, in many instances there is also overlap in pathology among different proteinopathies, complicating the diagnosis upon autopsy (Figure 2).

Although the clinical and pathological phenotypes that trigger neurodegenerative diseases are distinct, they share numerous similarities in terms of the molecular mechanisms involved, suggesting that the pathological cascades are likely to be more conserved that one might have anticipated.

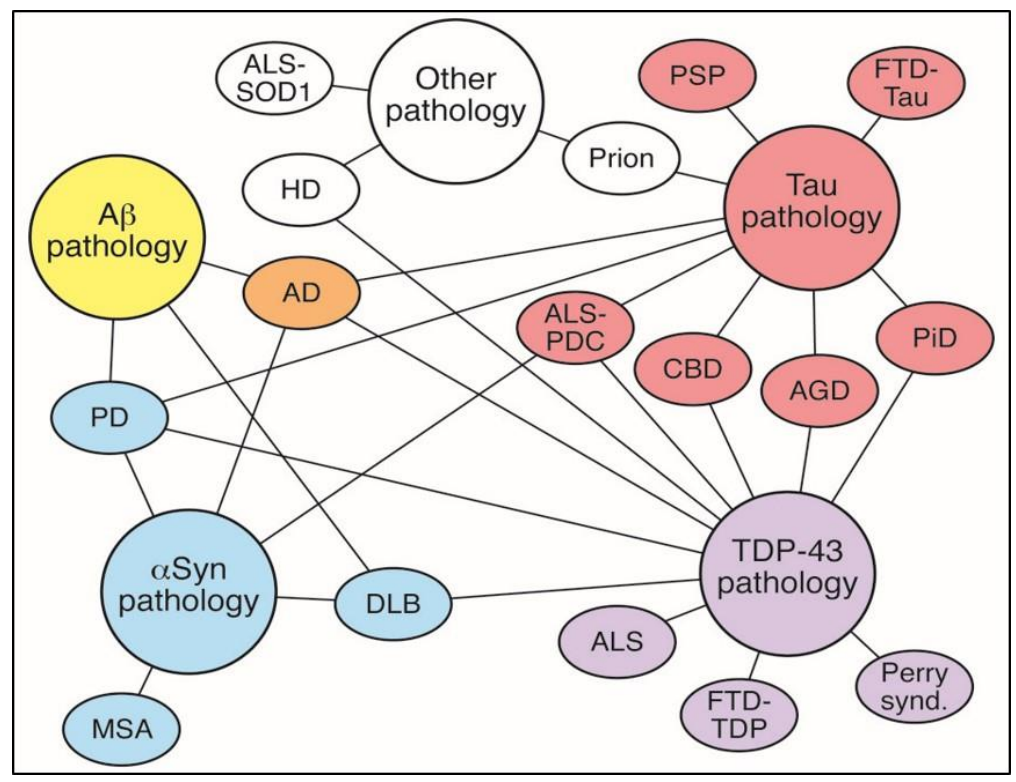

Figure 2. Overlap of neurodegenerative proteinopathies.

The different neurodegenerative disorders represented are arranged in colour blocks that indicate their primary proteinaceous aggregate. $\mathrm{AD}$ is characterized by proteinaceous inclusions of both $\mathrm{A} \beta$ (yellow) and tau (red) and is displayed in orange. Diseases are connected to the protein responsible of the formation of aggregates throughout the use of lines. (Abbreviation used: Argyrophilic grain disease(AGD); Corticobasal degeneration (CBD); Dementia with Lewy bodies (DLB); Frontotemporal dementia (FTD); Huntington's disease (HD); Multiple system atrophy (MSA); Perry syndrome (Perry synd); Pick's disease (PiD); Progressive supranuclear palsy (PSP). From Reference 15. 
The word "synucleinopathies" was adopted in order to characterize a subgroup of neurodegenerative disorders that share the accumulation of inclusions rich in the protein aSyn in certain populations of neurons or glial cells ${ }^{13,17-19}$. Synucleinopathies include PD, Dementia with Lewy bodies (DLB) and multiple system atrophy (MSA), as also other more maladies such as progressive supranuclear palsy, corticobasal degeneration, neurodegeneration with brain iron accumulation, type 1 , etc ${ }^{20-24}$.

\subsubsection{ASYN STRUCTURE}

aSyn is an abundant, 140 amino acid (aa) protein mainly localized in presynaptic terminals. The first sequence of aSyn was published in 1988 and it was obtained from the electric organ of the Torpedo californica ${ }^{25}$. The protein was called synuclein due to its localization in presynaptic nerve terminals (syn-) and in some part of the nuclear envelope (-nuclein). Following studies failed to confirm the nuclear localization of the protein, but the original name survived and, more recently, this topic has attracted renewed interest.

Although the precise function of aSyn remains elusive, extensive information have been accumulated about its structural properties and conformations.

Based on the amino acid composition, aSyn can be divided into three distinct regions:

- The N-terminal region (residues 1-60), contains four imperfect 11 aa repeats that bear the consensus hexameric sequence KTKEGV, where the individual repeats are separated by region of 5-8 aa. The $\mathrm{N}$-terminal region is predicted to form amphipathic $\alpha$-helical structures when associated with membranes ${ }^{26,27}$ due to its strong homology to the lipid-binding domains of class A2 apolipoproteins ${ }^{28}$.

- The central region (residues 61-95), also known as Non-Amyloid Component (NAC) domain, is highly hydrophobic and essential for aggregation ${ }^{29,30}$, and contains three additional KTKEGV repeats. The name of this domain is associated to the finding of a protein, named "non-amyloid beta $(\mathrm{A} \beta)$ component precursor", in some amyloid plaques from brains of patients with $\mathrm{AD}^{31}$. Even in this case, subsequent work failed to confirm the presence of NAC fragment in the amyloidogenic deposits of $\mathrm{AD}$ brains $^{32}$, but the name persisted for historical reasons.

- The C-terminal region (residues 96-140) is enriched in acidic residues and prolines (Pro), which confers to this domain a strongly negative charge. Three highly conserved tyrosine (Tyr) residues, which are considered a signature of the $\alpha$ - and 
$\beta$-synuclein family, are located on it. Increasing evidences suggest that this domain is essential for the chaperone function of $\mathrm{aSyn}^{33-35}$ and that may modulate the protein-protein and protein-small molecule interactions of $\mathrm{aSyn}^{36}$.

From a biophysical point of view, aSyn is a typical, intrinsically disordered protein (IDP). It is characterized by the lack of a well-defined secondary structure and exhibit significant conformational plasticity.

aSyn has not been crystallized, and the study of its structure has required the employment of sophisticated assays.

In Nuclear Magnetic Resonance (NMR) studies, the C $\alpha$ secondary chemical shifts analysis of aSyn confirmed that the protein is largely unfolded and that lack a defined structure $^{37,38}$. Nevertheless, NMR ${ }^{39-41}$, Electron Paramagnetic Resonance $(E P R)^{42}$, molecular dynamics ${ }^{40}$ and electron transfer studies ${ }^{43}$ shown that aSyn possess a relatively higher compact ensemble - mainly due to clustering of hydrophobic residues - compared to a fully unfolded peptide chain. Those studies highlighted as well the presence of transient long-range contacts between the C-terminal tail and the central region of the protein ${ }^{38,44}$, which were suggested to play a role in the inhibition of spontaneous aSyn oligomerization and aggregation ${ }^{38,45}$.

The intrinsically unfolded conformation exhibited under physiological condition can be altered both in vitro and in vivo, giving rise to a pre-molten globule state when aSyn is exposed to condition such as low $\mathrm{pH}$, high temperature, low concentrations of organic solvent or the presence of various salt and polications, as well as heparin and other glycosaminoglycans ${ }^{46}$.

A general preference for secondary structure in the form of $\alpha$-helices was found in the first 100 residues at the N-terminal - that show to have decreased mobility based on ${ }^{15} \mathrm{~N}$ relaxation rates measurement ${ }^{47}$ - leading to the formation of an extended, amphipathic $\alpha$-helix when aSyn binds to the membrane surface ${ }^{38}$.

Upon addition of small unilamellar vesicles (SUVs), it was reported a break in the helical pattern of the protein, between the residues 43 and 44, showing an interruption of the predicted structure ${ }^{26}$ and reporting the formation of two separated alpha-helices (corresponding to Val 3-Val 37 and to Lys 45-Thr 92) connected by an extended, ordered linker ${ }^{37,48}$. In both conformations, the acidic C-terminal region (from Asp 98 to Ala 140) behave as a disordered, flexible tail and remain unstructured even in the presence of membranes ${ }^{46,48}$.

As will be described in the next sections, aSyn is also able to form morphologically distinct oligomers and aggregates, containing both $\alpha$-helical and $\beta$-sheet secondary structures. The prolonged in vitro incubation of this protein at high temperatures, as 
well as with organic solvents over a long time results in a progressive aggregation through the formation of dimers and higher-order oligomers species containing possibly a rigid tertiary structure ${ }^{46}$.

The findings summarized above clearly show that aSyn belongs to the family of the IDPs, and more specifically, to the most disordered subfamily members, known as natively unfolded proteins: these are characterized by a unique combination of low hydrophobicity, low sequence complexity and high net charge $e^{38,43}$.

A

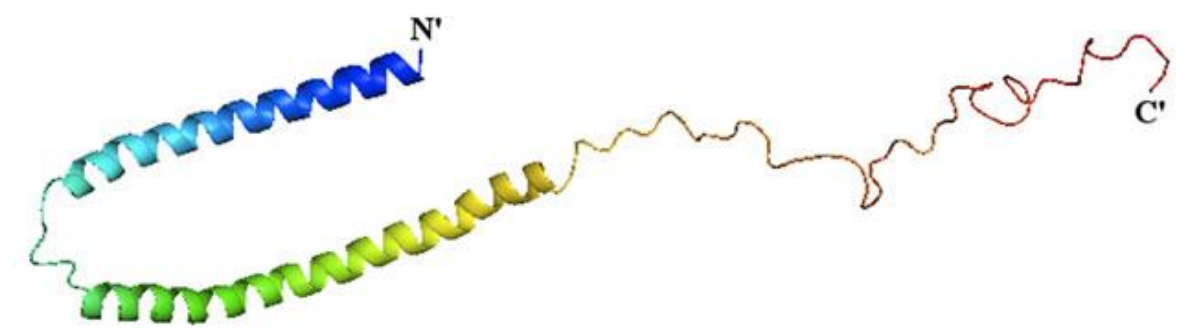

B

60

95 140

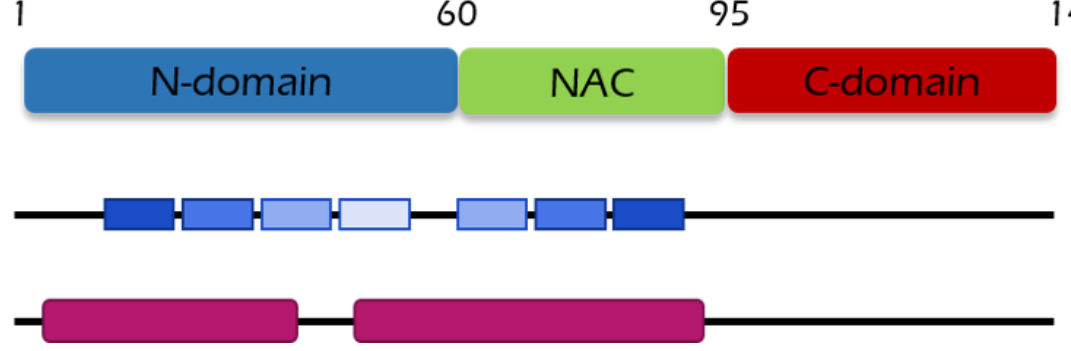

Figure 3. Schematic representation of the domains and structure of aSyn.

(A) Structure of aSyn bound to lipid vesicles. The structure of the protein (human, full length) was determined by NMR (PDB ID: 1XQ8). (B) Schematic illustration of the three structural domain of aSyn, the seven imperfect repeats (second lane) and the predicted alpha helices (third lane). Adapted from Reference 38. 


\subsection{ASYN: PHYSIOLOGICAL FUnCTION AND PATHOLOGICAL BEHAVIOUR}

\subsubsection{ASYN FUNCTION}

Although aSyn has been estimated to account for about $1 \%$ of the total cytosolic protein in the brain ${ }^{49}$, the exact function of this protein remains elusive.

For a long time it has been theorized that the predominant physiological state of aSyn was monomeric, and that the protein could be found either in a cytosolic, free state, either bound to plasma membrane and vesicles, where it acquire a more structured, $\alpha$ helical enriched conformation. Recently, multimeric forms of aSyn, mainly tetramers, have been isolated from human brains, neuronal and non-neuronal cultured cell lines ${ }^{50-}$ ${ }^{53}$, opening a debate about its natural state. Given the recent findings, one of the suggested model proposes that the tetrameric form of aSyn could actually be the functional form of the protein, and that this would exists in a regulated equilibrium with the monomers ${ }^{50-53}$.

The close association with membranes and vesicular structures encouraged several theories about the putative functions of aSyn, including transport of lipid, packing and stabilization of membranes, as well as regulation of phospholipids via the inhibition of phospholipase D2 $2^{50,54}$.

On the other side its localization at the presynaptic terminals ${ }^{49,55}$, its association with the pool of synaptic vesicles ${ }^{56,57}$ and the deficits in synaptic transmission observed after genetic manipulation of the SNCA gene, suggest that aSyn could be involved in synaptic plasticity, through the regulation of synaptic release and trafficking ${ }^{58}$. SNCAknockout mice show a reduced synaptic response in the hippocampus upon intense stimulation, as well as a decreased availability of synaptic vesicles, especially of the distal pool ${ }^{59}$.

In opposition, the overexpression of human aSyn in transgenic mice - as well as in cultured neurons - induce a decrease in ready-to-release vesicles and affects the recycling of those after endocytosis ${ }^{60}$. Excess of aSyn reduce the dopamine reuptake in nerve terminals and cause impairment in neurotransmitter release too ${ }^{61,62}$.

Overwhelming evidence suggest that aSyn may acts as a chaperone for the SNARE complex, regulating the degradation and affecting distribution, preservation and 
assembly of the proteins involved, which are directly implicated in docking and fusion, exocytosis of synaptic vesicles and neurotransmitters release ${ }^{63}$.

There are still some controversy about the interaction of aSyn with SNARE complex: a major study showed an increase in the assembly of SNARE complex in presence of $\mathrm{aSyn}^{63}$, while others demonstrated that aSyn inhibits vesicle fusion to membrane in a concentration-dependent manner, claiming for a negative regulatory role of aSyn ${ }^{64-66}$. A direct interaction of oligomeric aSyn with VAMP2 was reported, as a crucial step for the inhibition of the vesicle fusion process ${ }^{66}$, whereas other findings claim that a direct binding of aSyn to the complex is unnecessary and that the inhibition is merely due to the ability of aSyn to integrate into the lipid surface ${ }^{67,68}$.

Together these data draw a still imperfect picture of aSyn function in SNARE complex formation, but all of them also highlight a mechanism that relies on preservation of membrane curvature, stabilizing lipid packaging defects and preventing - seems likely - premature fusion of vesicles ${ }^{64,69}$.

The possible role of aSyn in regulating synaptic homeostasis, synaptic plasticity and trafficking is not exclusively related to its direct interaction with vesicles and with the proteins of the SNARE complex; aSyn has been show to interact with at least 30 different proteins and other ligands, as well as with several polyvalent elements including $\mathrm{Fe}^{2+}, \mathrm{Zn}^{2+}, \mathrm{Cu}^{2+}$ and $\mathrm{Ca}^{2+} \cdot 33,70,71$ (Figure 4).

aSyn also binds to calmodulin, a protein that regulates secretory processes at the synapses by interacting with the calcium-CaM dependent kinase II and other protein targets $^{72-75}$. The interaction with calmoduline contribute to the release of membranebound aSyn, suggesting that dissociation of aSyn from the membrane surface ${ }^{74}$ occurs through a depolarization-dependent process ${ }^{72}$.

In addition to these various, supposed functions, aSyn might also be involved in dopamine synthesis. It has been widely reported that aSyn binds to and inhibits tyrosine hydroxylase, an enzyme necessary for the biosynthesis of dopamine, as well as to $\mathrm{hDAT}$, (human dopamine transporter) and that it prevent the re-uptake of dopamine from synapsis ${ }^{72,76}$.

Key interactions have been localized to particular fragments of aSyn, showing that the majority of them are contained within the C-terminal region. All these mapped interactions could possibly be affected by familial mutations in the protein and by posttranslational modifications (PTMs), and they may be involved in the diverse function and dysfunction of the protein, contributing or maybe even causing its pathological behaviour. 


\subsubsection{FAMILIAL MUTATIONS}

aSyn started to attract significant interest in 1997, after a single point mutation in the SNCA gene was found to be associated with familial cases of early-onset $\mathrm{PD}^{14,38}$. The same year, Spillantini and colleagues identified a strongly immunoreactivity for aSyn in LB inclusions present in cases of sporadic $\mathrm{PD}^{20}$.

Since then, several observations have confirmed and established the involvement of aSyn and its aggregated, misfolded forms in the pathogenesis of PD and other synucleinopathies. Few mutations in the SNCA gene have been identified (A53T ${ }^{14}$, $\mathrm{A} 30 \mathrm{P}^{77}, \mathrm{E} 46 \mathrm{~K}^{78}$ and most recently $\mathrm{H}_{50 \mathrm{Q}^{79}}, \mathrm{G} 51 \mathrm{D}^{80}$ and $\mathrm{A} 53 \mathrm{E}^{81}$ ) and overexpression of wild-type aSyn (aSyn WT) due to duplication ${ }^{82}$ and triplication ${ }^{83}$ of the SNCA gene similarly led to autosomal dominant PD. Therefore, the behaviour and properties of these mutants have been studied in details in the attempt to find a recurring motif and to shed new light on the mechanisms of aSyn pathogenesis ${ }^{72}$.

aSyn contribution to neurodegenerative disorders could be caused either from a toxic gain of function (as result of overexpression and disruption of the functional pathways), or from the perturbation of its natural, physiological role (as consequence of familial mutations and PTMs, or from sequestration into misfolded, aggregated forms $)^{72}$.

To note, the effects of the familial mutations on the aggregation properties vary considerably. E46K, H50Q and A53T accelerate the kinetics of the secondary structure changes responsible for oligomerization and fibril formation ${ }^{84,85}$; A30P oligomerize rapidly but delays the formation of mature fibrils ${ }^{85-87}$ while G51D retards aggregation ${ }^{84}$, suggesting that the process that bring toxicity involved are different ${ }^{72}$.

Different mutants have also dissimilar effects on membrane binding affinity ${ }^{72}$ : compared to aSyn WT, A30P is clearly less effective on bind to the membrane, A53T has no effect, and E46K shows enhanced binding ${ }^{88-91,92}$. Nevertheless, a common characteristic in the membrane binding behaviour of these mutants is showed through their ability to adopt a partly folded, $\alpha$-helical enriched structure, where the first 25 aa at the $\mathrm{N}$-terminal are bound and the rest of the protein remains mostly free $\mathrm{e}^{72}$. The H50Q and G51D mutations have been discovered in recent times and there are not extensive studies yet. However, G51D reduces the affinity for membranes, while H50Q does not show altered membrane binding properties ${ }^{72,84,93}$. 


\subsubsection{Post-TRANSLATIONAL MODIFICATIONS}

Multiple PTMs of aSyn have been reported, including serine/threonine (Ser/Thr) and Tyr phosphorylation ${ }^{94}$, ubiquitination ${ }^{95}$, sumoylation ${ }^{96}$, glycation ${ }^{97}$, acetylation ${ }^{98}$ and methionine oxidation ${ }^{99,100}$.

The most common, disease-related PTMs of aSyn is the Ser phosphorylation of the residue 129 , identified as a major, highly conserved phosphorylation site, followed by a second one located at Ser 87 . It has been estimated that a significantly elevated fraction ( $>90 \%$ ) of aSyn deposits in LBs is phosphorylated at Ser 129, and that this modification promotes the formation of aggregates ${ }^{101-103}$.

LBs analysed from PD and DLB patients and cytoplasmic inclusions in MSA are abundantly loaded with ubiquitinated aSyn as well. Since ubiquitination is not required for the degradation of monomeric aSyn, it appears that this PTMs occurs after its aggregation, and although aSyn contains 15 lysine (Lys) residues, only Lys 6, 10 and 12 were shown to be ubiquitinated in vivo ${ }^{95,104}$.

It was recently reported that aSyn is $\mathrm{N}$-terminally acetylated, most likely by the $\mathrm{N}$ terminal acetyltransferase $\mathrm{B}$ complex $(\mathrm{NatB})^{105}$, and that such a modification slightly increases the helical propensity of the first 10 residues in the $\mathrm{N}$-terminal region ${ }^{72,106,107}$, although additional studies are required to better clarify the exact role of this modification. Further studies demonstrated that aSyn is acetylated on Lys 6 and 10 and that these residues are deacetylated by Sirtuin2, proposing aSyn acetylation as an important regulatory mechanism for aSyn aggregation and toxicity ${ }^{98}$. 


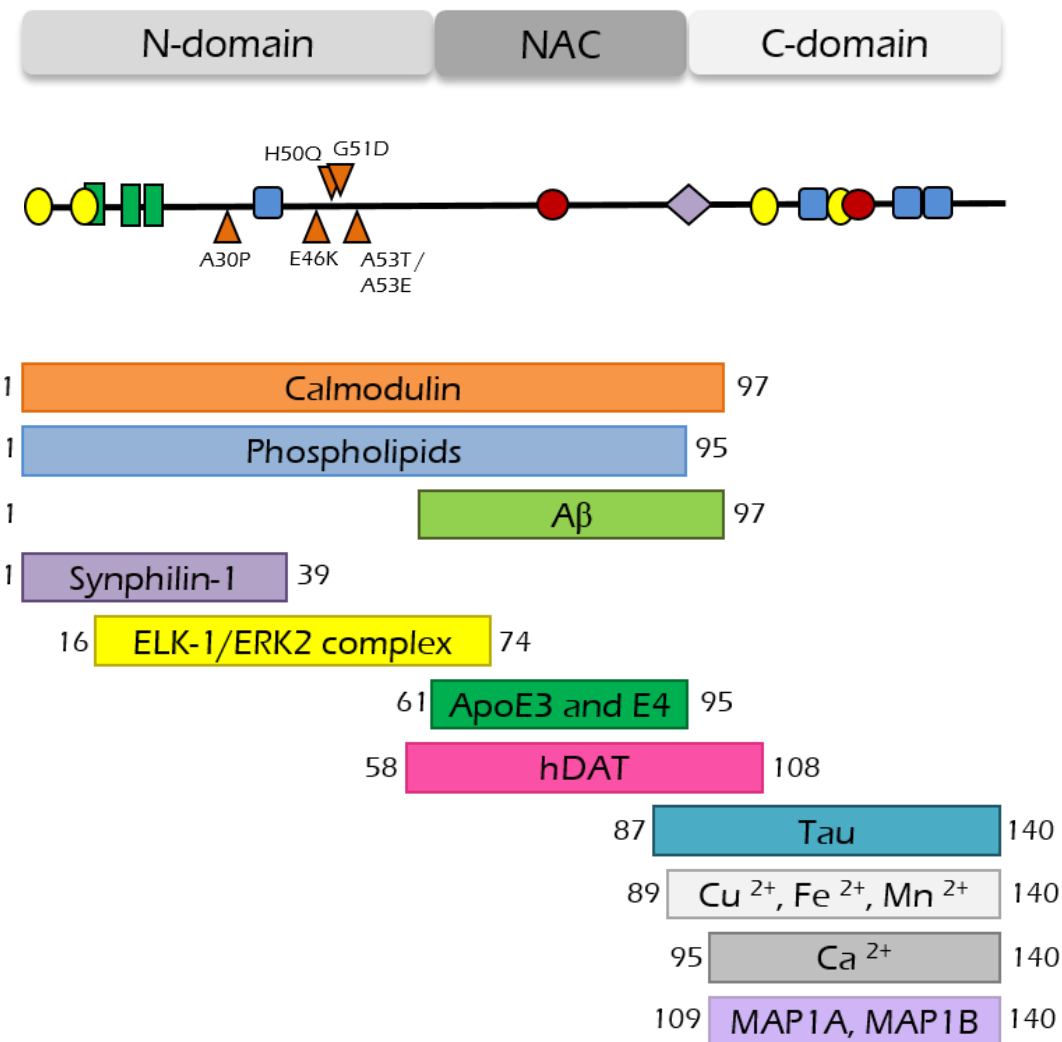

Figure 4. Schematic representation of aSyn familial mutation, PTMs and map of the interactor protein binding site. (A) Representation of aSyn structure with the three characteristic structural domain (B) Sites of PTM (methionine: yellow ovals; Tyosine: blue squares; phosphorylation: red rings; sumoylation: purple squares; ubiquitination: green boxes;) and PD-related familial mutations (orange arrowheads). (C) Representation of the interaction domains responsible of the protein-protein and protein-small molecules interaction of aSyn. Modified from Reference 38. 


\subsubsection{The Putative Pathogenic Role OF ASyn}

Due to the clear, widespread accumulation of $\beta$-sheet enriched amyloid fibrils in the brains of patients with synucleinopathies, intense effort had focused on the study of the molecular mechanisms underlying aSyn misfolding and aggregation, as well as on the structural and morphological characterization of these fibrils.

As for many other proteins, the levels of aSyn in the central nervous system (CNS) depend on the delicate equilibrium between aSyn synthesis, aggregation and clearance: an imbalance between these mechanisms can result in abnormal levels of aSyn and therefore in the formation and/or accumulation of oligomers and fibrils.

It seems also reasonable to suggest that due to the structural polymorphism and the numerous interaction and function ascribed, aSyn is a protein potentially prone to misfold per se. We could look at the misfolding and aggregation of aSyn as a low rate, stochastic event that occurs throughout life. Under some stress conditions (i.e. toxic insults, inflammation, cellular stress) this process is promoted. Normally, neurons are able to clean up accumulated, dysfunctional proteins, but it is also possible that in some occasions the cellular clearance mechanisms fail, letting the pathogenic process start. Fibril formation is a multistep process, and several studies tried to better understand the specific pathways involved in aSyn aggregation, as well as the toxic contribution of any and all the species originated along such pathways, from monomeric aSyn, to oligomers, prefibrillar aggregates, and mature amyloid fibrils (Figure 5).

Early stages of this process involve the partial folding of aSyn into a pre-molten globule-like conformation. High protein concentration, point mutations, oxidative damages and many other environmental factor may shift the equilibrium in favour of this partially folded conformation ${ }^{108}$. In the process of oligomerization, dimers are formed first ${ }^{109}$, followed by a series of morphologically different - spherical, chain-like and annual - soluble oligomers.

In vitro, under appropriate conditions, spherical oligomers convert into ring-like structures $^{110}$ and protofibrillar species, that have been reported to permeabilize membranes through the formation of pores or channels ${ }^{72,111,112}$. This could clearly alter membrane potential and ion distribution, actively contributing to cellular toxicity ${ }^{72}$. Finally, the protofibrillar species can assemble into large, insoluble aggregates by adopting two different morphologies: amorphous aggregates and amyloid-like fibrils. The formation of aSyn fibrils occurs in a nucleation- dependent manner, where the limiting step is the growth of transient, oligomeric intermediate, also called fibril nuclei ${ }^{38,113}$. Once fibril nuclei have formed, fibrils grow from these seeds by a "dock 
and lock" mechanism, in which monomers, initially bind to - or "dock onto" - an already formed fibril in a reversible manner ${ }^{38}$. This step is therefore followed by an , structural reorganization of the polypeptide, in order to generate an optimal area for further fibril growth ${ }^{38}$.

An important question regarding the pathobiology of aSyn in neurodegenerative disorders is which one, among the one involved in the fibrillization of aSyn, are the toxic species.

Giving the fact that aggregation of aSyn and accumulation of this into insoluble deposits occur after cell death in vitro ${ }^{114}$ and that toxicity is usually documented without the presence of heavily aggregated aSyn ${ }^{115}$, it seems reasonable to suggests that the soluble, oligomeric intermediates - rather than mature fibrils - might be the key contributors to cellular dysfunction and death.

The mechanisms proposed to describe the neurotoxic events in synucleinopathies can be divided into three major groups: physical disruption of cellular processes or cellular compartments, toxic gain of function and loss of physiological function ${ }^{38}$. Those factors are not necessarily mutually exclusive: they could be, instead, synergistic ${ }^{38}$.

One of the most commonly accepted mechanisms of disruption of cellular processes is the permeation of cellular membranes. As mentioned before, in vitro studies were able to demonstrate that annular protofibrils are capable of modulating membrane permeability, enhancing the influx of calcium from the extracellular to intracellular space and resulting eventually in cell death ${ }^{116}$.

aSyn oligomers might also cause toxicity by damaging mitochondria ${ }^{117}$, causing lysosomal leakage ${ }^{118}$ or disrupting microtubules ${ }^{119}$ (Figure 6).

In light of the potential interaction of aSyn with the SNARE complex and RAB proteins, it is not surprising that overexpression and oligomerization of aSyn could affect the transport machinery, resulting in endoplasmic reticulum (ER) stress and cytotoxicity and blocking ER to Golgi trafficking ${ }^{120}$. aSyn oligomers impair as well the axonal transport of synaptic proteins, resulting in dysfunctional synapses and neurodegeneration ${ }^{58}$.

Impairment of aSyn degradation via proteasome inhibition and generation of reactive species of oxygen (ROS) have been proposed as another possible, neurotoxic mechanism.

However, the previous proposed mechanisms do not excludes the other possibilities: on one side, the oligomerization process may perturb the distribution of physiological, functional species of aSyn, by trapping monomers into non-functional oligomers and therefore leading to a partial loss of function of the protein ${ }^{121}$. 
On the other side, intrinsically existing, misfolded forms of monomeric aSyn can play a role in the pathogenesis of synucleinopathies through some sort of aggregationindependent mechanism, possibly triggered by aberrant interactions with proteins, membranes and small molecules, or by misplacement of aSyn species in incorrect physiological compartments and by disruption of cellular processes, resulting in a gain of toxic function ${ }^{121}$.

It is worth to mention that unlike stable oligomeric forms of aSyn, which can be easily analysed and characterized, the existing experimental tools do not allow a detailed, structural characterization of different monomeric forms of aSyn. This strongly limits the study of the role of aSyn monomers in health and disease ${ }^{121}$.

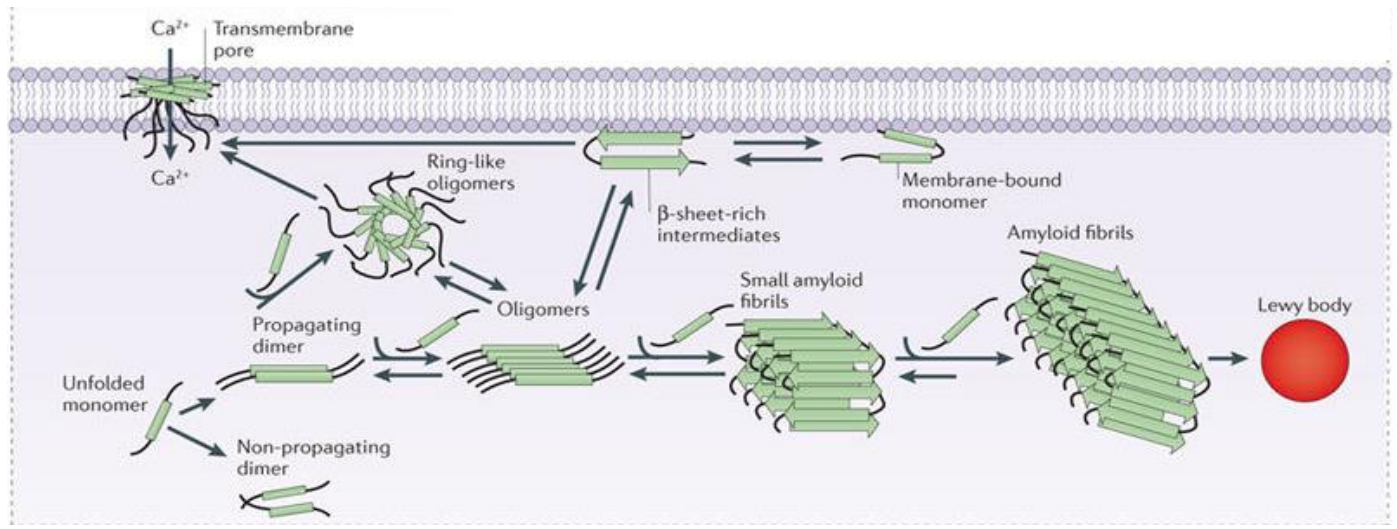

Figure 5. Mechanisms of aSyn aggregation.

In the cytosol, aSyn unfolded monomers start to interact and to create dimers that slowly aggregate to generate oligomers with different morphologies. aSyn oligomers eventually convert to fibrils via an irreversible, nucleation-dependent mechanism. The accumulation of these amyloidogenic fibrils bring to the formation of Lewy bodies. The ring-like oligomers may associate with membranes, creating trans-membrane pores. Both aSyn oligomers and fibrils can be transferred from one cell to another and induce the spreading of the pathology to other brain regions. Modified from Reference 121. 


\section{Physiological function}

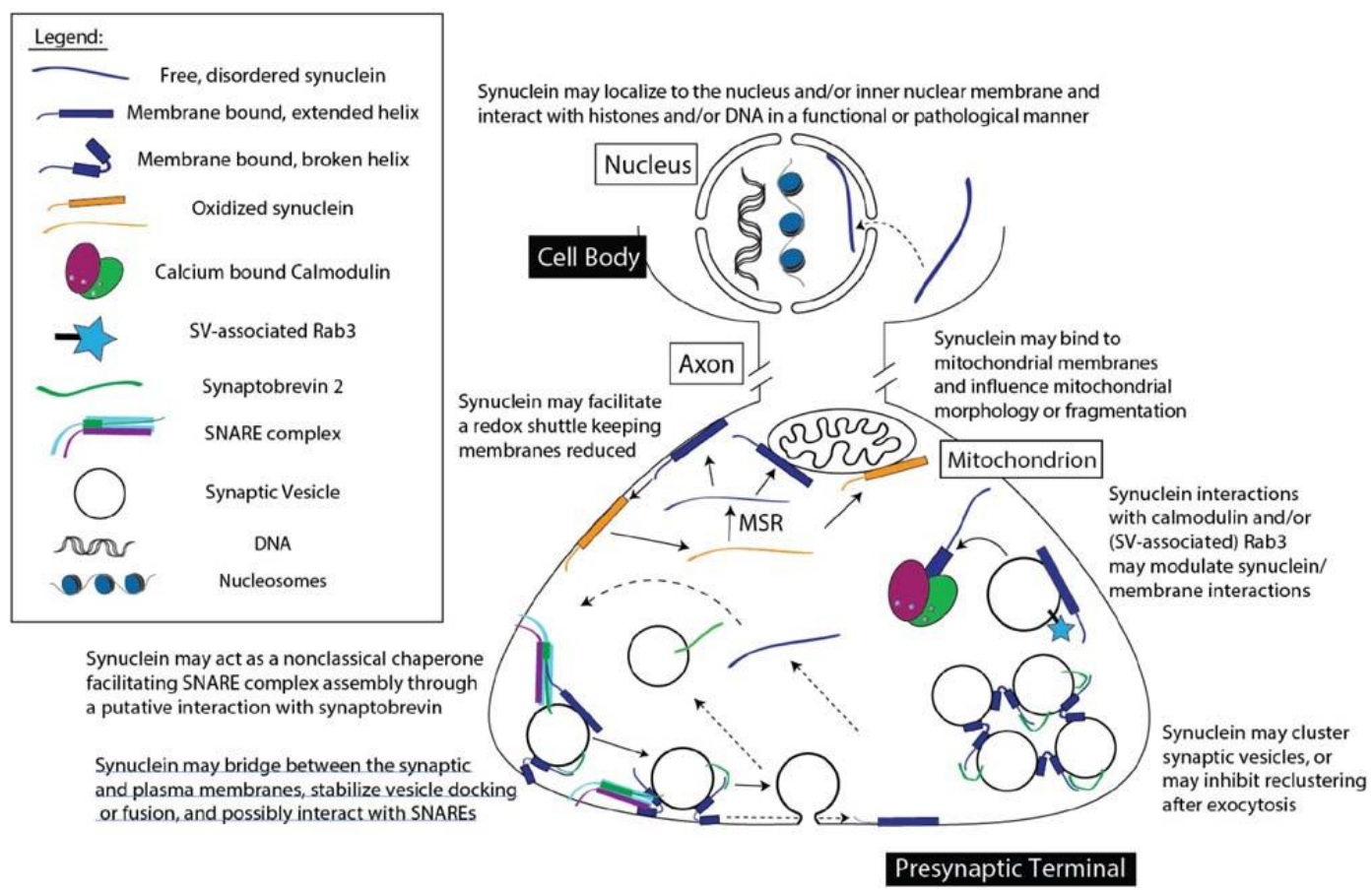

\section{Pathological behaviour}

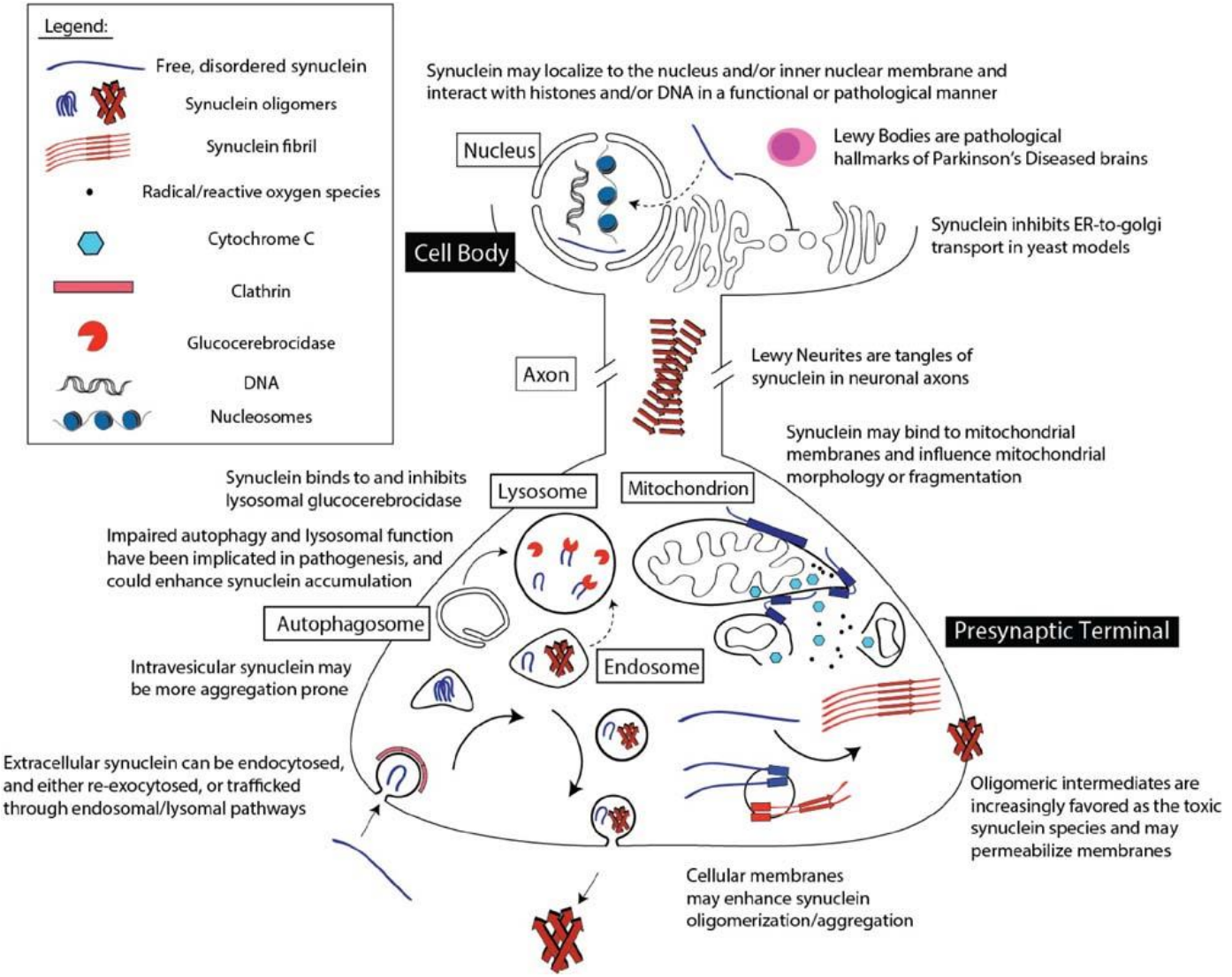

Figure 6. aSyn physiological function and pathological behaviour.

Pathways, membranes and targets potentially involved in the normal, physiological function of aSyn (top figure) and in aSyn dysfunction and its role in synucleinopathies (bottom figure).

Adapted from Reference 72. 


\subsubsection{ASYN: A PRION-LiKE PROTEIN?}

Recently, the possibility that the progression of aSyn pathology in synucleinopathies would be mediated through cell-to-cell spreading of oligomers or aggregates has become an attractive model to explain how aSyn related toxicity and neurodegeneration might propagate throughout neuroanatomically connected regions of the diseased brain.

Braak and colleagues hypothesized about the progressive, stereotypic spread of Lewy pathology by histopathological studies of PD post-mortem brains ${ }^{11}$. In the early stage of the disease, aSyn positive LB and LNs accumulate mainly in the olfactory bulb and in the dorsal motor nucleus of the vagus and then they spread in anatomically connected regions with the progression of the disease ${ }^{50,122}$ (Figure 7).

Six neuropathological stages of PD were described, with increasing number of regions that successively exhibit aSyn pathology, proposing a model where a "spreading agent" (e.g. a neurotrophic virus) might propagate through a defined neuronal pathway, in a prion-like manner ${ }^{123}$.

A supportive observation to this theory resulted from an experimental treatment in which embryonic dopaminergic neurons were transplanted into the putamen of human PD patients ${ }^{124,125}$. The finding of LB-like inclusions in these exogenously grafted neuronal population significantly influenced the field, leading to the formulation of the prion-like hypothesis of aSyn, which postulated that misfolded aSyn is transferred between connected cells, acting as a template to initiate aggregation of endogenous protein in recipient and otherwise healthy neurons $\mathrm{s}^{50,126,127}$.
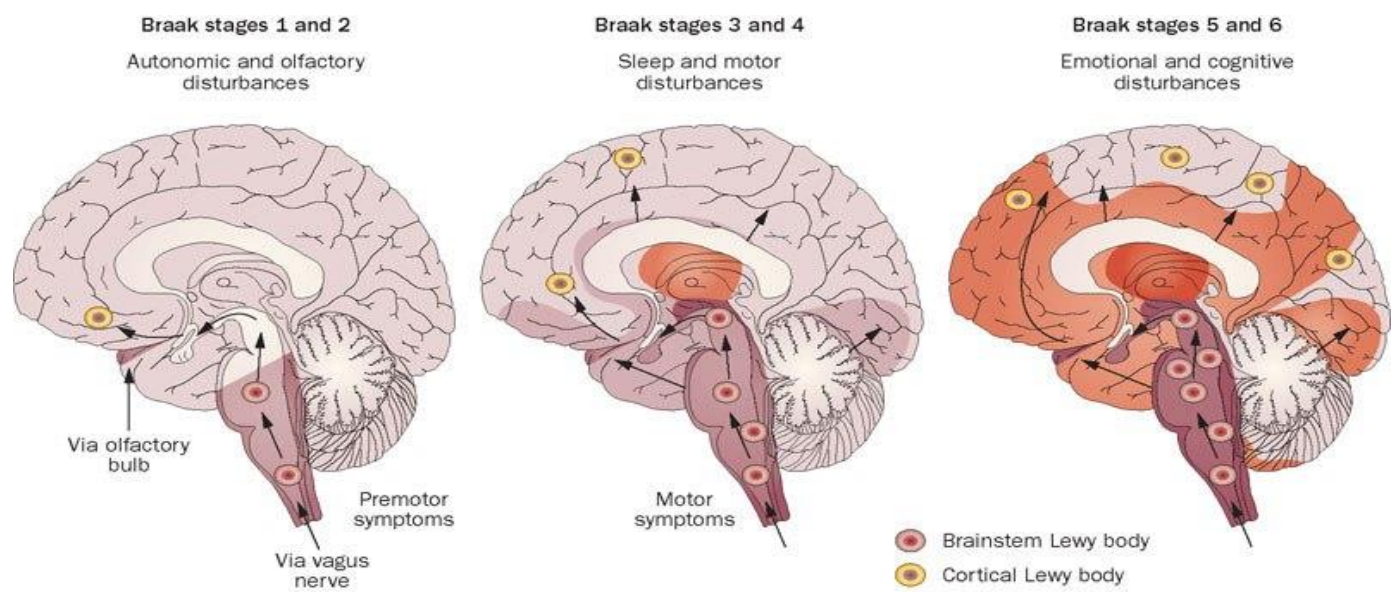

Figure 7. Braak's staging hypothesis for the progression of PD pathology.

Variable red shading reflects the ascending, rostro-caudal progression of the disease and increasing severity of aSyn pathology. From Doty RL, Olfactory dysfunction in Parkinson disease, Nat Rev Neurol. 2012; 8(6):329-39. 
A key premise for the prion-like hypothesis is that aSyn assemblies can be taken up by neurons, transported along the axons and finally transferred to neighbouring cells through different ways, (i.e. being released into the extracellular space) ${ }^{128}$.

So far, numerous in vitro and in vivo studies have addressed different aspects of this complex series of events ${ }^{80,128-136}$.

First, it has been widely demonstrated that exogenously added aSyn oligomers and aggregates can bind to the surface of cultured cells, both in their free state or associated in extracellular vesicles, due to the interaction with membranous proteins ${ }^{137,138}$, or with lipid components of the membranes ${ }^{139}$ (i.e., proteoglycans).

Several mechanisms for the internalization of aSyn have been proposed, some of them appearing to be assembly-state specific. Monomeric aSyn seems to be able to penetrate cell membranes and passively diffuse into cells ${ }^{129}$, while larger assemblies utilize specific pathways, including receptor-mediated endocytosis ${ }^{139}$.

It has been shown that exogenous aSyn further induced neuronal cell death through Rab 5A-dependent endocytosis ${ }^{140}$, while another study reported that heparan-sulphate proteoglycans mediate macropinocytosis of aSyn and other aggregation prone proteins ${ }^{139}$.

Several findings showed that aSyn oligomers and fibrillary species can enter cells via dynamin-dependent endocytosis, and that absorptive endocytosis (an intermediate process between fluid-phase endocytosis and receptor-mediated endocytosis) promoted the uptake of both tau and aSyn fibrils ${ }^{50,134,141}$. However, due to the size of fibrillar aggregates, seems unlikely to consider receptor-mediated endocytosis - which requires the interaction between ligands and cell-surface receptors - as a major mode of fibril internalization ${ }^{142}$.

Based on the above-mentioned mechanisms, it looks like a direct cell contact is not necessarily required for the propagation of aSyn aggregates. Nonetheless, recent evidence suggests that tunnelling nanotubes could also be involved in the spreading of $\mathrm{aSyn}^{143}$. These structures would provide a useful channel for the migration from one cell to another, obviating the need for those misfolded proteins, otherwise enclosed within an endosome, to cross plasma membrane and to gain access to the cytosolic compartment (Figure 8).

Once inside the naïve cells, exogenous, misfolded aSyn assemblies can amplify by recruiting and triggering the aggregation of endogenous, cytosolic aSyn ${ }^{128}$. Many efforts in the last decade were aimed at shedding light into the process responsible of the structural conversion of aSyn, suggesting that endogenous aSyn aggregates through a seeding process where the imported aSyn act as a template. The intrinsic 
structure of the seed is preserved by structurally well-defined longitudinal and lateral molecular interaction between newly recruited aSyn monomers and the terminal, exposed part of the seed, through the use of a "lock and dock" mechanism, as described above.

Part of the aSyn assemblies can also be degraded by lysosomes ${ }^{129}$, which is particularly interesting considering that dysfunction and impairment of the autophagy-lysosomal pathway (ALP) are strictly linked to PD pathogenesis.

Transmission of aSyn fibrils from neuron to neuron can undergo anterograde and retrograde transport, and some of the monitored movement of aSyn assemblies occurs at a velocity consistent with fast axonal transportation ${ }^{135}$.

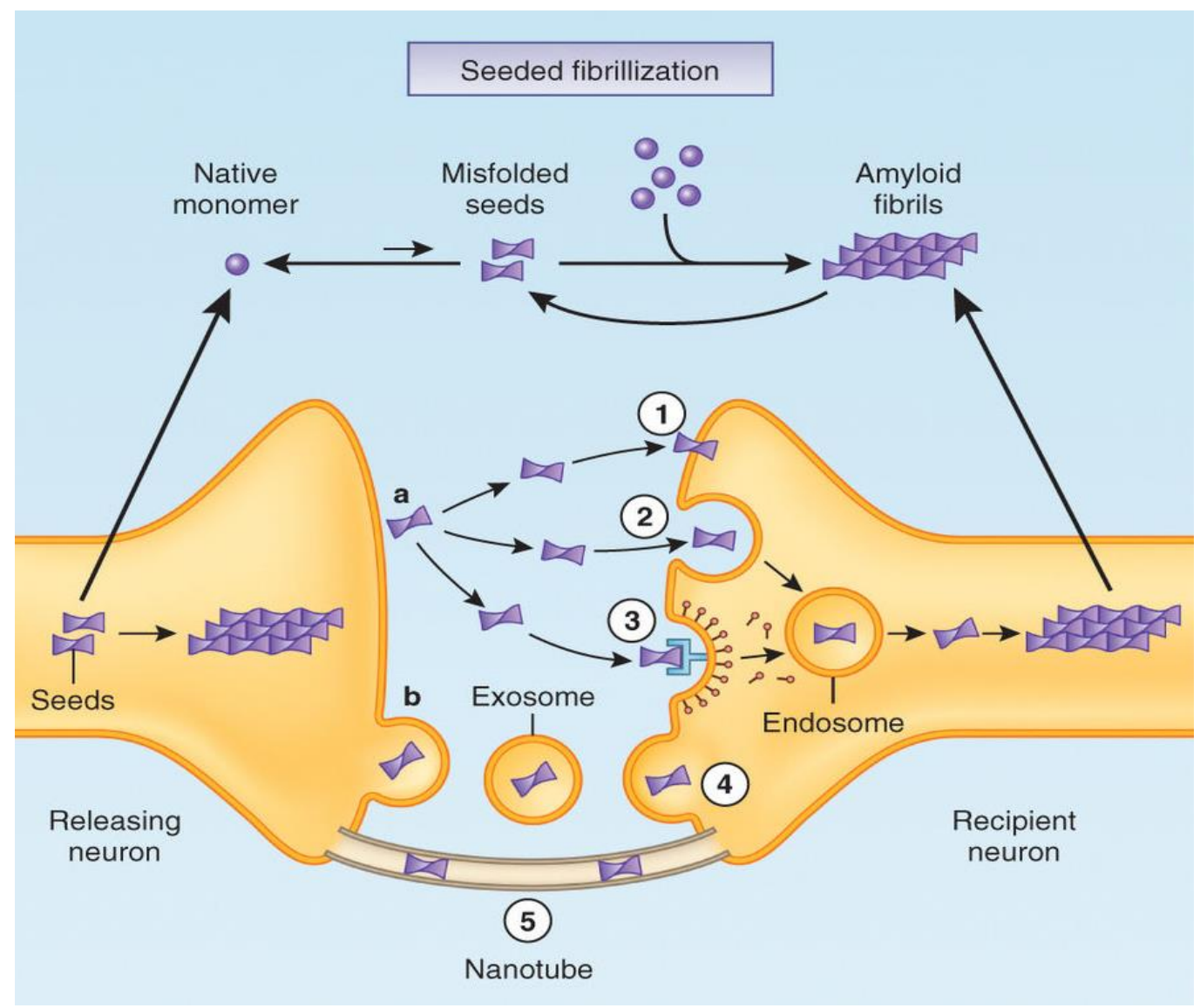

Figure 8. Potential proposed mechanisms mediating cell-to-cell transmission of aSyn.

Misfolded protein seeds in the form of oligomers or protofibrils are initially formed in the cytoplasm of the donor neuron, where soluble monomers are recruited into larger aggregates. A large number of propagating seeds can be generated through fragmentation of the existing fibrillary species, or through secondary nucleation. Protein aggregates can be released in the extracellular space in a "naked" form (a) or via exosomes (b). aSyn can passively diffuse trough the plasma membrane or (1) enter the recipient cell by fluid-phase endocytosis (2) or receptor-mediated endocytosis, (3) as well as through the fusion of exosomal vesicles with the recipient cell. (4) The transfer of protein could also happen via nanotubes, structures that connect directly the cytoplasm of two cells (5). Internalized, misfolded protein then recruit native monomers in the recipient neuron, repeating the nucleation process and allowing the spreading of pathology. Adapted from Reference 142. 
Regarding the release of aSyn, intracellular aSyn aggregates can be secreted into the extracellular space and therefore uptaken by neighbouring neurons, microglia or astrocytes ${ }^{133,135,136}$.

The first report highlighting the detection of aSyn in the extracellular space even preceded the prion-like propagation hypothesis, and was based on the observation of aSyn molecules in biological fluids - including cerebrospinal fluid (CSF) and blood plasma - of both healthy and PD subjects ${ }^{144}$. These results imply that aSyn is typically available in the extracellular space in the CNS, regardless of a pathological state.

Further results obtained from cultured cells confirmed that aSyn can be secreted in an active way and that the secretion can be constitutive or regulated ${ }^{50}$. Furthermore, higher cytoplasmic level of the protein - as well as inhibition of the proteasomal and lysosomal system ${ }^{128}$ - lead to an increased release of aSyn, probably due to some compensative mechanism necessary to keep balanced the cytosolic level of the protein. Pathways leading to the release of toxic aSyn oligomers include exocytosis in clear vesicles $^{129}$, exosomal release ${ }^{145,146}$ and penetration from the donor cell membrane $e^{121}$. Another way through which aSyn can reach the extracellular moiety is throughout necrotic cell death, although there are still very little evidences suggesting that the amount of aSyn released from these cells and its contribution to the extracellular, pathological pool would be determinant for the propagation of the disease ${ }^{50}$.

Overall, cellular stress, proteasomal and mitochondrial dysfunction ${ }^{131}$ as well as overexpression and cytosolic accumulation seems to drive aSyn secretion from neural cells, supporting the general idea that aSyn exocytosis may increase in the affected brains, bringing a fundamental contribution to the disease progression.

Another essential process for the prion-like hypothesis is the propagation of misfolded aSyn and the spreading of the related neuropathology in different, distant brain region. This was shown in multiple experiments in rodent and nonhuman primate, using intracerebral injection of brain homogenates from PD and DLB patients, brain tissues from transgenic animal with aSyn pathology, and preparation of pre-formed fibrils (PFFs) generated from recombinant aSyn ${ }^{141,147-149}$.

Injection of aSyn PFFs into the striatum not only lead to aggregation of endogenous aSyn, but it also had drastic consequences on the viability of dopaminergic neurons, leading to a widespread synaptic dysfunction ${ }^{134}$. Of note, few months after the injection aSyn aggregates had spread throughout the brain, while mice injected with vehicle remained healthy and free of pathology, indicating that the presence of misfolded, exogenously added aSyn is sufficient to trigger and spread aSyn pathology $y^{150}$. 
One of the advantages of using recombinant protein is that the seeding process can be controlled carefully in the test tube before the intracellular injection is made, which does not apply to a crude mix such a brain homogenate.

Indeed, one of the latest debate in the field is related to the existence of different "strains" of aSyn aggregates and whether their presence could explain the different morphological conformation - as well as the cellular and anatomical predilection - of aSyn inclusions in different synucleinopathies ${ }^{151}$.

Intracerebral or systemic injections of fractionated brain homogenates from PD or MSA patients in animal models induce distinct neuropathology with "strain-specific" features and characteristic that resemble the pathology of origin, adding further support to the idea that different types of aSyn fibrillary assemblies exhibit different toxicity and give rise to different type of neuropathology. 


\subsection{ASYN, MEMBRANES AND CELLULAR TRAFFICKING}

\subsubsection{ASYN AND MEMBRANES}

In presynaptic termini, monomeric aSyn exist in a tightly regulated equilibrium between soluble and membrane/vesicle bound states, with approximately $15 \%$ of the protein associated to the membrane.

Upon binding to phospholipidic bilayers, aSyn shows a dramatic increase in $\alpha$-helix content, promoting the interaction of the protein with a wide variety of different lipid assemblies. This membrane-induced disorder-to-order transition is driven by a specific amino acidic pattern in the $\mathrm{N}$-terminal region of the protein and it has been widely characterized ${ }^{72}$.

Many distinct helical conformation have been detected, spanning from amphipathic helices that lie down along the phospholipidic surface, with their apolar surfaces deeply inserted into the membrane and some of the lysine residues "snorkelling" from the inner side of the membrane in order to interact with the lipid headgroups ${ }^{72}$.

A switching between an extended and a broken helical structure can be initiated by changes in the ratio of lipid binding partners or by modifying the curvature of the binding surface displayed by micelles, lipid vesicles or SDS-flat bilayers ${ }^{152}$ (Figure 9).

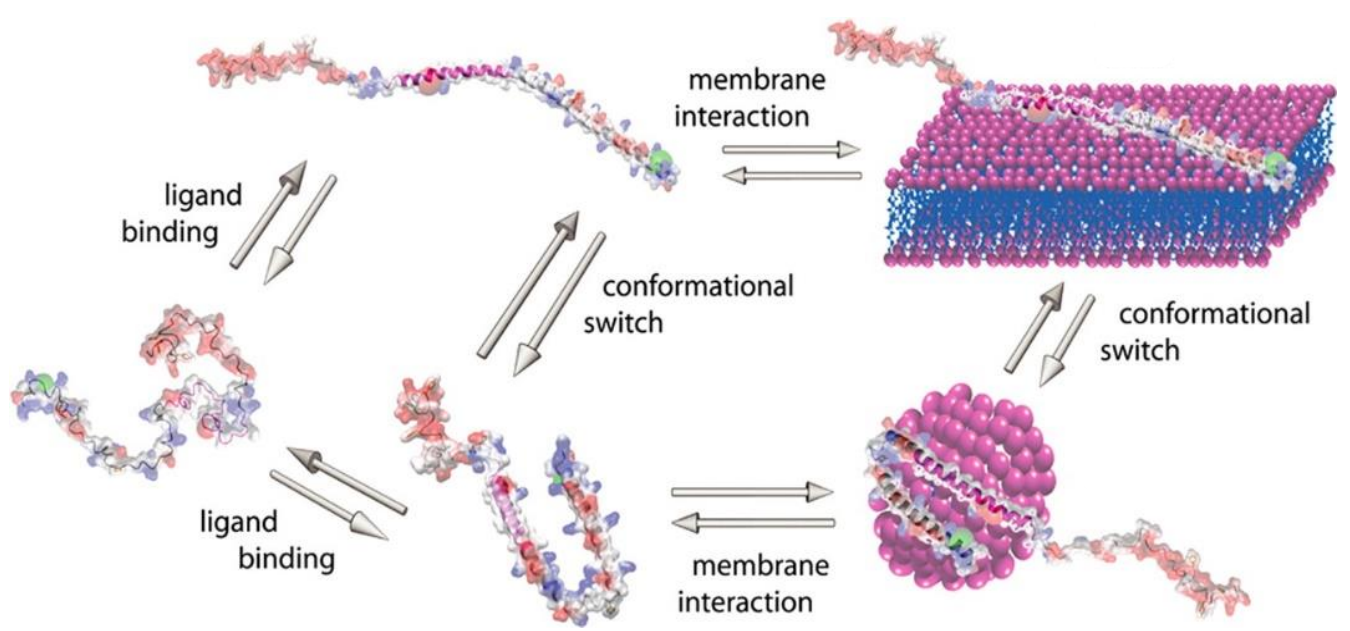

Figure 9. Proposed model for aSyn folding and conformational switching induced by binding to small-molecules ligand and stabilizing membrane mimic surfaces. Adapted from Reference 152 
In the extended helical conformation, aSyn binds to the membranes with the first 100 residues at the $\mathrm{N}$-terminal, with an unusual $11 / 3$ periodicity ${ }^{27,152,153}$, while in the broken helix conformation two distinct, antiparallel curved $\alpha$-helices are separated by a nonhelical linker region spanning from residues $39-45^{26,27,48}$. Additional binding modes observed in the context of phospholipid vesicles include shorter helices involving the first 25 residues at the N-terminus, while the remainder of the domain stay unbound. Finally, a recent NMR-based study ${ }^{154}$ revealed the exact amino acid residues involved in the membrane binding process (Figure 10). The findings show that the binding of aSyn to the membranes generate three dynamically different structures in distinct regions of the protein. The first 25 residues at the $\mathrm{N}$-terminal are categorised as the membrane "anchor" domain, binding tightly to the membrane and enhancing cooperatively the binding of the central region as well. The central portion (from residues 26 to 98) includes also the NAC fragment; this bind less tightly and it play a key role in the modulation of the affinity of aSyn for cellular membranes. To finish, the C-terminal region remains mostly unstructured and uncoupled with membranes. Distinct conformational states are certainly contributing to the putative functions of aSyn, although a detailed, mechanistic understanding of its behaviour at the membrane surface is still an open path of research.

The interaction with membranes may interfere with the pathogenic behaviour of aSyn too, either through the effect of aggregated, misfolded protein on the integrity and structure of membranes itself, either through a more direct effect on aSyn aggregation: depending on the protein: lipid ratios, membrane binding features could either have a protective role against further aggregation by sequestering aSyn monomers, either favour aSyn oligomerization and aggregation by increasing the effective cytosolic concentration of this protein on a bidimensional surface ${ }^{72}$.

As a consequence of its metamorphic character, aSyn is also able to sense membrane curvature and defects, showing an enhanced binding in relation to an increased curvature $^{155,156}$. This may be related to the abundance of packing defects in highly curved membranes ${ }^{72}$. This so called packing defects are portions of the membranes where the acyl amphipathic chains of the phospholipids are momentarily exposed, resulting in effective protein binding sites ${ }^{68,155-157}$. Furthermore, current structurefunction relationship suggests that not only aSyn is attracted to highly curved membranes, but that can generate and stabilize membrane curvature itself, as part of its physiological function. 
To this end, the presynaptic terminal can be considered as a hot spot for membrane remodelling, as synaptic vesicles continuously reach the active zone to release their neurotransmitter content. On their side, synaptic vesicles are considered the most important membrane-binding target of aSyn in the cell, and are markedly enriched in small packaging defects: the membrane curvature stabilizing function of aSyn could therefore have an effect on vesicles trafficking and release. In addition to this, aSyn could promote these processes via its double-anchor mechanism: by binding to lipid membranes with the N-terminal region (aa 1-25) and additionally with the central region (aa 65-97), aSyn would be able either to fuse two vesicles to one another, or vesicles to the plasma membranes, facilitating the processes for exo and endocytosis.

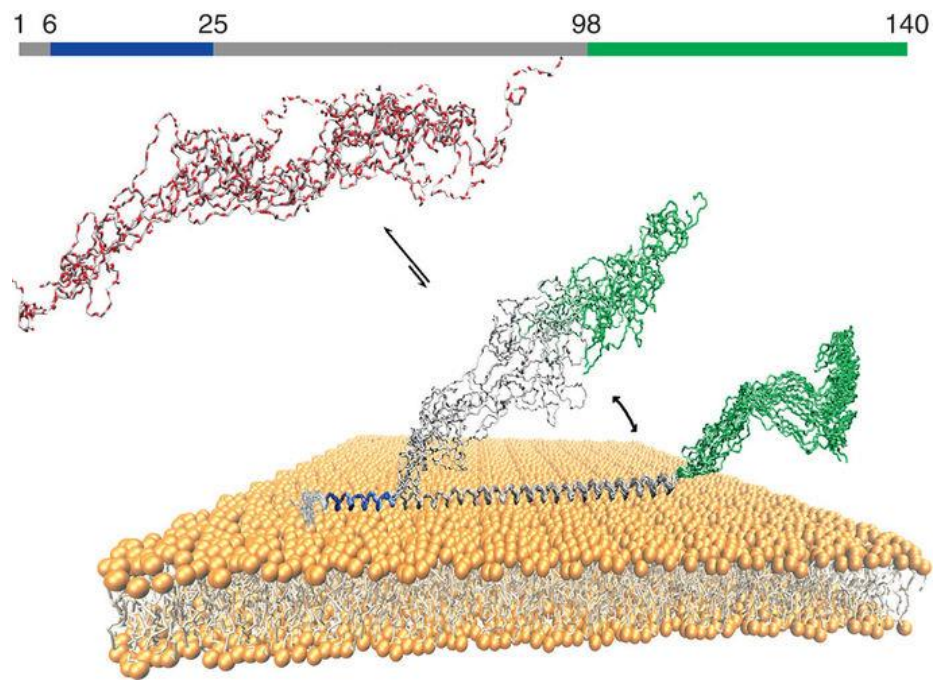

\subsubsection{ASyn And The RAB GTPases FAMily Proteins}

Presynaptic neurotransmitter release is a process that requires the biogenesis, fusion and turnover of vesicles and involves remodelling and movement of membranes and other molecules. In this context, small GTPases from the Rab family proteins (RABs) play a crucial role as key mediators in several steps and processes related to the membrane trafficking pathways, by interacting with and regulating different macromolecular complexes in each different stage of the cycle ${ }^{158}$.

Notably, mutations affecting RABs and their effectors and regulators have been associated to several neurodevelopmental and neurological diseases. A series of recent studies revealed that certain RABs are involved in the modulation of aSyn and that the impairment of these proteins could be one of the rare causes for inherited early onset 
$\mathrm{PD}^{159-161}$. At the same time, it has also been demonstrated that the pathological accumulation of aSyn perturbs the homeostasis of RABs as well.

Whether there is a direct interaction between RABs and aSyn or a general compensatory mechanism linked to the vesicle transportation deficits is something that need still to be clarified, and the present work will bring some additional information about the role of RABs and the related pathways involved in the internalization and in the metabolism of aSyn.

RABs constitute the largest family of small Ras-like GTPases, serving as molecular "switches" that alternate between two conformational states: the GTP-bound 'on' form and the GDP-bound 'off' form ${ }^{162}$. They are evolutionarily conserved across eukaryotic cells, with more than 60 members in humans, classified in several phylogenetic and functional groups ${ }^{163,164}$ (Table A).

As all the other members of the Ras superfamily, RABs exhibit a GTPase structure, with six-stranded $\beta$-sheet and five $\alpha$-helices; the carboxi-terminal of the GTPase fold is the hypervariable region of RABs and it is followed by CAAX boxes containing two cysteine (Cys) residues that facilitate the reversible association of RABs to the membranes after prenylation ${ }^{158,161,162,165}$

Nucleotide cycling of RABs is tightly coupled to their membrane association/ dissociation cycle. Once transported to the membrane, RABs are activated by the exchange of GDP for GTP, a process catalysed by a guanine nucleotide exchange factors (GEFs) ${ }^{166,167}$. The GTP-bound active conformation can therefore interact with multiple effector proteins, and is converted back to the GDP-bound inactive form by GTPase-activating proteins (GAPs), which accelerate RAB-GTP hydrolysis. RABGDP dissociation inhibitor (RAB GDI) recognize the GDP-bound RAB and sequesters it in the cytosol until it is recruited to a membrane again, a process that requires the interaction of a GDI displacement factor (GDF) $)^{158,162}$.

The conversion of the GDP "off" form into the GTP "on" form involves remarkable conformational changes in two regions named switch I and switch II: those are located in the surface of the RABs, and bind to the interactor proteins ${ }^{168}$. Despite the structural similarity, the switch regions possess a degree of variety that confers unique specificity to each individual RABs in order to interact with their corresponding effectors and pathways, therefore creating an great variety in RABs function and localization ${ }^{158}$ (Table A). Indeed, RABs are essential for the definition of the identity of subcellular membranes, and trafficking organelles possess their own peculiar set of RABs controlling their formation, maturation, and transition as well as their interaction with other compartments ${ }^{158}$ (Figure 11). 


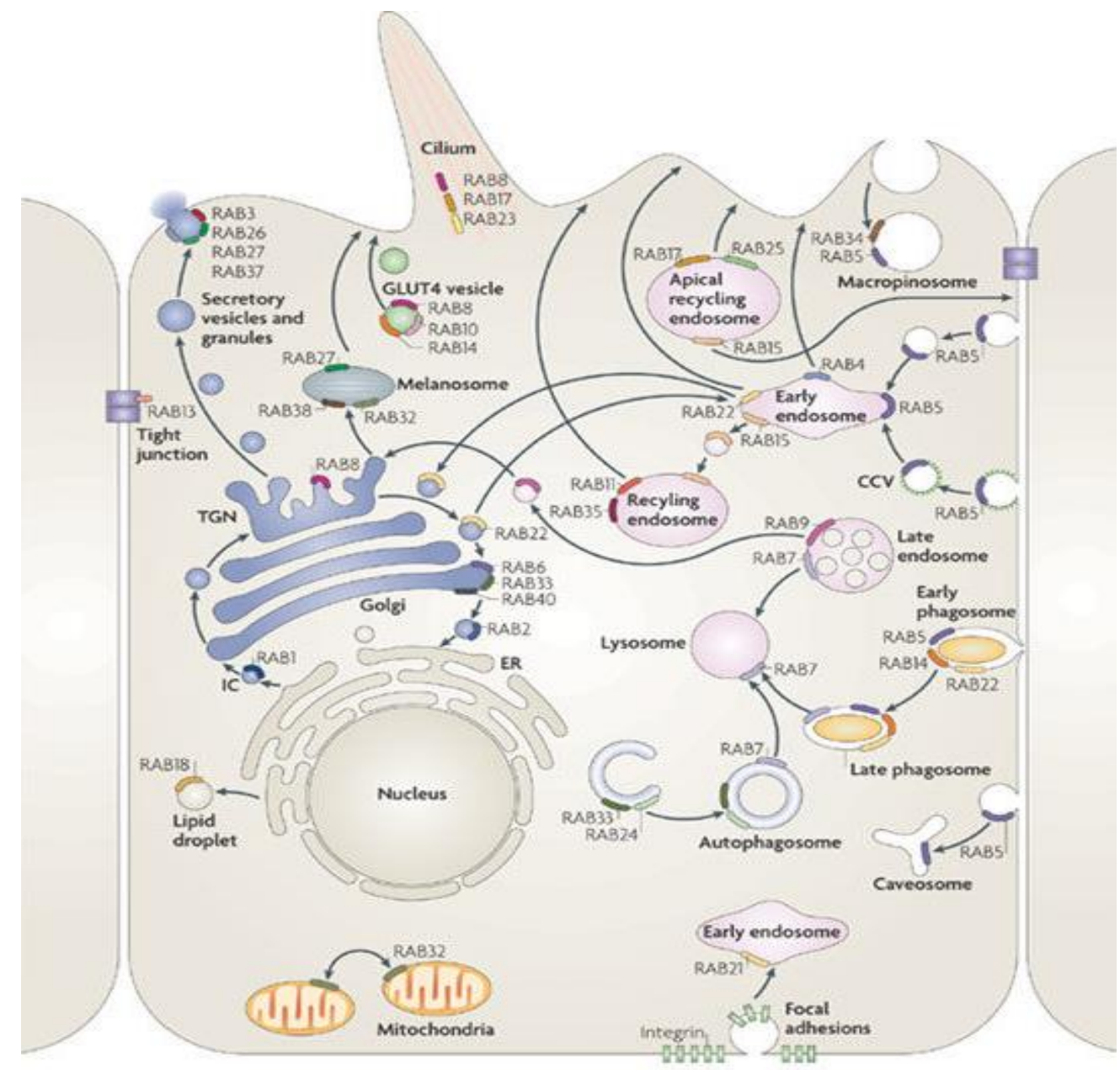

Figure 11. Localization and function of RABs

An epithelial cell with its vesicle transport pathways and the localizations of selected Rab GTPases. From Reference 162.

A large number of studies have tried to clarify the interactions between RABs and aSyn, and this can be addressed from two different perspectives.

On one side, several RABs (Rab 3A, Rab 5, Rab 8, Rab 7 and Rab 11A) were shown to have the ability to interact with aSyn and to protect cells from the toxicity and the gain of pathological function induced by its overexpression or by mutated forms. This was demonstrated throughout co-immunoprecipitation and immunofluorescence studies in different cellular and animal models ${ }^{169,170}$.

In control brains, Rab $3 \mathrm{~A}$ appears to be associated only weakly with soluble, monomeric aSyn, while in DLB brains aggregates of aSyn sequester Rab 3A and prevent its interaction with rabphilin ${ }^{171}$.

An NMR spectroscopy study revealed that the C-terminal region of aSyn binds to the switch region of Rab 8A, that play a role in the Golgi and in the Trans-Golgi Network 
(TGN) trafficking. In line with a direct Rab 8A/aSyn interaction, Rab 8A enhanced aSyn aggregation and reduced aSyn-induced cellular toxicity ${ }^{172}$.

Finally, a shRNA-based screening additionally confirmed that Rab 8, Rab 11A, and $\mathrm{Rab} 13$ act as modulator of aSyn oligomerization and reduce the toxicity induced by misfolded aSyn trough the enhancement of secretion in cultured cells $\mathrm{s}^{173}$.

On the other side, RABs were shown to regulate the distribution and recycling of aSyn, as well as in other vesicular trafficking pathways connected to the lysosomal degradation and the release of this protein.

As a regulator of synaptic vesicles, it was reported that Rab 3A have a close association with aSyn, and that its activity may play a role in the re-distribution of aSyn and the modulation of its presynaptic activity ${ }^{174}$.

Using a drosophila model of aSyn toxicity, it was reported that overexpression of Rab11 could significantly reverse the neuromuscular synaptic potentiation by modulating synaptic vesicle size. Furthermore, Rab 11 decreases aSyn aggregation and enhances several aSyn-dependent phenotypes in both larvae and adult flies, including muscular activity and neurodegeneration ${ }^{175}$. Rab 11 also contribute to maintain the proteostasis of aSyn and its level: in addition to the degradation that occurs through lysosomes, once internalized aSyn might be secreted or released in the extracellular space via exocytosis, a process facilitated by the overexpression of this Rab-GTPases family member ${ }^{169}$.

Additional evidences for aSyn/RABs interaction came from extended genetic analyses in the yeast $S$. cerevisiae ${ }^{176}$. Although yeast does not have an aSyn orthologue, it has been used widely for elucidating the mechanism of aSyn toxicity in a general, cellular contest and to test potential therapeutic targets ${ }^{177,178}$.

It has been demonstrated that one of the processes affected in the very first step, as a result of the overexpression of aSyn in yeast is related to the ER-to-Golgi transport. aSyn accumulation was indeed responsible for the failure of the processes of tethering and fusion to Golgi membranes, leading to the accumulation of morphologically undocked vesicles near the plasma membrane and eventually in intracellular vesicle clusters, in a both dose and time dependent manner ${ }^{176,179}$. In this context, YPT1, the yeast orthologue of mammalian Rab 1 was shown to associate with aSyn aggregates and to rescue trafficking defects and aSyn-related toxicity ${ }^{176}$. Furthermore, transgenic overexpression of Rab 1 protected against the dopaminergic neuron loss induced - or related - to aSyn in three different models - drosophila, $C$. elegans and cultured rat midbrain neurons ${ }^{178,179}$. 
Further studies have also shown that aSyn aggregation affects the retrograde transport, by perturbing the endosome to Golgi trafficking machinery pathway as well ${ }^{180}$.

Recently, Rab 35 was identified as a potential biomarker for the diagnosis of PD and functional studies also suggested that overexpression of Rab 35 promote the aggregation and release of aberrant aSyn ${ }^{161,181}$.

In addition, genetic analyses recently identified that defect in Rab 39B lead to pathologically defined $\mathrm{PD}$, reporting that altered Rab 39B was quickly degraded by the proteasome system, causing mislocalization of $\mathrm{aSyn}^{160}$. According to the latest findings, Rab 39B might be involved in the autophagy regulation ${ }^{161,182}$.

Taken together, these findings suggest that the aSyn spreading and pathology may be attributed, at least in part, to the deregulation of a subset of RABs.

aSyn overexpression could induce extensive defects in vesicular trafficking, and in particular in the neurons and in the synaptic buttons, where the downstream events such as docking and fusion - are affected more extensively ${ }^{178}$. This could be explained perhaps through the ability of aSyn oligomers or aggregates to interact in a deregulate way with RABs, causing dysfunction in the related pathways due to the inability of the GTPases to effectively engaging with their effectors ${ }^{178}$. This inhibition may provoke the pathological accumulation of unpacked, dysfunctional vesicles and the consequential impairment of the synaptic activity ${ }^{178}$. 


\begin{tabular}{|c|c|c|c|}
\hline RAB Protein & Localization & $\begin{array}{l}\text { Membrane Trafficking } \\
\text { Pathway/Function }\end{array}$ & Effector Proteins; Related Diseases \\
\hline Rab 1A; Rab 1B & ER, Golgi & ER to Golgi, intra-Golgi & $\begin{array}{l}\text { p115/Uso1, GM130, giantin, golgin-84, } \\
\text { GCC185, MICAL-1, MICAL-COOH terminal } \\
\text { like, JRAB/MICAL-L2, OCRL1, INPP5B, } \\
\text { Cog6, GBF1 (Arf1 GEF), Iporin. }\end{array}$ \\
\hline Rab 2A; Rab 2B & $\begin{array}{l}\text { ER, ER-Golgi intermediate } \\
\text { compartment, Golgi }\end{array}$ & ER to Golgi & $\begin{array}{l}\text { INPP5B, golgin-45, RIC-19, PKC iota/lambda, } \\
\text { GM130, GAPDH, Drosophila melanogaster cell- } \\
\text { less homolog 1, GARI, Fam71f2, Fam71b }\end{array}$ \\
\hline Rab 3A; Rab 3B; Rab 3C & $\begin{array}{l}\text { Secretory vesicles, plasma } \\
\text { membrane }\end{array}$ & $\begin{array}{l}\text { Exocytosis, neurotransmitter } \\
\text { release }\end{array}$ & $\begin{array}{l}\text { Rabin3, RIM1a, RIM2a, granuphilin, Noc2, } \\
\text { Munc18-1, rabphilin, INPP5B, SNAP-29, } \\
\text { synapsin, polymeric IgA receptor (Rab3B), Gas8 } \\
\text { (Rab3B), Zwint-1 (Rab3C), ORCL1; Warburg } \\
\text { Micro/Martsolf syndromes (Rab3GAP) }\end{array}$ \\
\hline $\operatorname{Rab} 3 \mathrm{D}$ (Rab 16) & $\begin{array}{l}\text { Secretory vesicles, plasma } \\
\text { membrane }\end{array}$ & $\begin{array}{l}\text { Exocytosis, regulated exocytosis } \\
\text { in nonneuronal cells }\end{array}$ & \\
\hline Rab 4A; Rab 4B & Early Endosome & $\begin{array}{l}\text { Portein recycling/transport to } \\
\text { plasma membrane }\end{array}$ & $\begin{array}{l}\text { CD2AP, D-AKAP2, Rabip4, Rabaptin-5(a), } \\
\text { Syntaxin 4, Dynein LIC-1, Rab coupling protein } \\
\text { (RCP), Rabenosyn-5, Ar11, BIG1, BIG2, Arf1, } \\
\text { Arf3. }\end{array}$ \\
\hline Rab 5A; Rab 5B; Rab 5C & PM, CCVs, early endosome & Early endosome fusion & $\begin{array}{l}\text { EEA1, Rabaptin-5(b), Rabex-5, Rabenosyn-5, } \\
\text { INPP5B, OCRL1, PI3 kinases, Rabankyrin-5, } \\
\text { APPL1, APPL2, Huntingtin-HAP40, caveolin-1, } \\
\text { angiotensin II type 1A receptor }\end{array}$ \\
\hline Rab 6A; Rab 6B & Golgi & $\begin{array}{l}\text { Endosome to Golgi, intra-Golgi } \\
\text { transport, Golgi to ER }\end{array}$ & $\begin{array}{l}\text { Rab6A interacting protein 1/2A/2B, Cog6, } \\
\text { kinesin Rab6-KIFL, GCC185, giantin, OCRL1, } \\
\text { ELKS, INPP5B,, golgin-97, golgin-245, } \\
\text { DYNLRB1, p150 subunit of dynein/dynactin } \\
\text { complex, mint3 adaptor protein, Bicaudal D1/2, } \\
\text { VFT complex, golgin Sgm1; Gerodermia } \\
\text { osteodysplastica (golgin SCYL1BP1). }\end{array}$ \\
\hline Rab 7 & $\begin{array}{l}\text { Late endosomes, } \\
\text { lysosomes/vacuole, } \\
\text { melanosomes, phagosomes }\end{array}$ & Late endosome to lysosome & $\begin{array}{l}\text { Vps } 35 / 29 / 26 \text { complex (retromer), Rabring } 7 \text {, } \\
\text { proteasome alpha-subunit PSMA7, Vps } 24 / \mathrm{p} 150, \\
\text { PI3-kinase complex, oxysterol binding protein } \\
\text { related protein 1, RILP; Charcot-Marie-Tooth. }\end{array}$ \\
\hline Rab 8A; Rab 8B & Cell membrane, vesiscles & $\begin{array}{l}\text { Exocytosis, TGN/RE to plasma } \\
\text { membrane }\end{array}$ & $\begin{array}{l}\text { Rabphilin-1, MICAL-1, MICAL COOH-terminal } \\
\text { like, MICAL-L1, JRAB/MICAL-L2, TRIP8b } \\
\text { (Rab8B), FIP-2, optineurin, otoferlin, RIM1, } \\
\text { RIM2, Noc2, OCRL1, cenexin3; Bardet-Biedel } \\
\text { syndrome, Hungtington's disease. }\end{array}$ \\
\hline Rab 9A; Rab 9B & Late endosomes, Golgi (9B) & Endosome to TGN & $\begin{array}{l}\text { TIP47, INPP5B, GCC185, PI3P PIKfyve kinase } \\
\text { associated protein p } 40 \text {, NdeI, 14-3-3 protein } \\
\text { theta, HPS4 }\end{array}$ \\
\hline Rab 10 & $\begin{array}{l}\text { Golgi, basolateral sorting } \\
\text { endosomes, GLUT4 vesicles }\end{array}$ & $\begin{array}{l}\text { Exocytosis, TGN/RE to plasma } \\
\text { membrane }\end{array}$ & $\begin{array}{l}\text { Rim1, MICAL-1, MICAL COOH-terminal like, } \\
\text { MICAL-L1, JRAB/MICAL-L2 }\end{array}$ \\
\hline Rab 11A; Rab 11B & Golgi, RE, early endosomes & TGN/RE to plasma membrane & $\begin{array}{l}\text { Sec15, RAB11-FIP1 to FIP5, D. melanogaster } \\
\text { nuclear fallout, arfophilin-2, myosin Vb, PI4- } \\
\text { kinase } \beta \text {, rabphilin-11, Rab6 interacting protein 1, } \\
\text { Rabin3, Sec2 (Ypt31/32), Gyp1 (Ypt32); } \\
\text { Hungtington's Disease. }\end{array}$ \\
\hline Rab 12 & Golgi, secretory vesicles & Exocytosis & $\begin{array}{l}\text { Rab interacting lysosomal protein-like } 1 \\
\text { (RILP-L1) }\end{array}$ \\
\hline Rab 13 & Cell/tight junctions, TGN, RE & TGN/RE to plasma membrane & $\begin{array}{l}\text { MICAL-1, MICAL COOH-terminal like, } \\
\text { MICAL-L1, JRAB/MICAL-L2, protein kinase } \\
\text { A, INP5B, OCRL1 }\end{array}$ \\
\hline Rab 14 & $\begin{array}{l}\text { Golgi, early endosome, GLUT4 } \\
\text { vesicles }\end{array}$ & $\begin{array}{l}\text { TGN/RE to plasma membrane; } \\
\text { apical membrane targeting }\end{array}$ & FIP2, RCP, Rip11, D-AKAP2 \\
\hline Rab 15 & Early/sorting endosome, RE & $\begin{array}{l}\text { Sorting endosome/RE to plasma } \\
\text { membrane }\end{array}$ & $\begin{array}{l}\text { MICAL-1, MICAL COOH-terminal like, } \\
\text { MICAL-L1, JRAB/MICAL-L2, Rab15 effector } \\
\text { protein }\end{array}$ \\
\hline Rab 17 & $\mathrm{RE}$ & Transcytosis & \\
\hline Rab 18 & Golgi, lipid droplets & Lipid droplet formation & \\
\hline Rab 19 & Golgi & Unknown & $\begin{array}{l}\text { D-AKAP2, dGolgin } 97, \text { Wdr } 38 \text {, oxidative stress- } \\
\text { induced growth inhibitor family member } 2 \text {. }\end{array}$ \\
\hline $\operatorname{Rab} 20$ & Golgi, endosome & Apical membrane recycling & INPP5E \\
\hline
\end{tabular}




\begin{tabular}{|c|c|c|c|}
\hline RAB Protein & Localization & $\begin{array}{l}\text { Membrane Trafficking } \\
\text { Pathway/Function }\end{array}$ & Effector Proteins; Related Diseases \\
\hline Rab 21 & Early endosome & Endosomal transport & Alpha-Integrin subunit \\
\hline $\mathrm{Rab} 22 \mathrm{~A}$ & Early endosome & $\begin{array}{l}\text { Endosomal transport, protein } \\
\text { recycling to plasma membrane }\end{array}$ & $\begin{array}{l}\text { Rabex-5, EEA1, rabenosyxn-5, RAD51, } \\
\text { INPP5B, OCRL1, rKIAA1055 }\end{array}$ \\
\hline Rab 23 & PM, endosome & $\begin{array}{l}\text { Protein recycling/transport to } \\
\text { plasma membrane }\end{array}$ & Carpenter syndrome \\
\hline Rab 24 & ER & Autophagosome formation & $\mathrm{COOH}$-terminal binding protein 1 \\
\hline Rab 25 & $\mathrm{RE}$ & $\mathrm{RE}$ (apical) to plasma membrane & $\begin{array}{l}\text { Integrin-beta1 subunit, FIP2, RipI1; epithelial } \\
\text { cancers }\end{array}$ \\
\hline Rab 26 & Secretory granules & Exocytosis & RIM1 \\
\hline Rab 27A; 27B & Melanosomes & Exocytosis & $\begin{array}{l}\text { S1p1-5, Slac2-a (melanophilin), Slac-2b, } \\
\text { granuphilin, Slac2-c, Rim2, Rabphilin, Noc2, } \\
\text { Munc13-4, Golga4/p230; Griscelli syndrome }\end{array}$ \\
\hline Rab 28 & Unknown & Unknown & Unknown \\
\hline Rab 30 & ER, Golgi & Unknown & Cog4, Golga4/p230, dGolgin97, dGolgin245 \\
\hline Rab 31 (Rab 22B) & TGN, endosome & $\begin{array}{l}\text { M6P receptor transport to } \\
\text { endosome }\end{array}$ & OCRL1 \\
\hline Rab 32 & Mitochondira, melanosomes & $\begin{array}{l}\text { TGN to melanosomes, } \\
\text { mitochondrial fission }\end{array}$ & Varp/Ankrd27, PKA \\
\hline $\mathrm{Rab} 33 \mathrm{~A} ; \mathrm{Rab} 33 \mathrm{~B}$ & Golgi, dense-core vesicles & Autophagosome formation & ATG16L, GM130, Rabaptin-5, Rabex-5 \\
\hline Rab 34 & Golgi, macropinosomes & $\begin{array}{l}\text { Intra-Golgi transport, peri-Golgi } \\
\text { positioning of lysosome }\end{array}$ & Hmunc13, RILP, RILP-L1 \\
\hline Rab 35 & PM, endosome & $\begin{array}{l}\mathrm{RE} \text { to plasma membrane, actin } \\
\text { assembly }\end{array}$ & $\begin{array}{l}\text { MICAL COOH-terminal like, MICAL-L1, } \\
\text { MICAL-1, OCRL1, fascin, Centaurin } \beta 2\end{array}$ \\
\hline Rab 36 & Golgi & Unknown & $\begin{array}{l}\text { MICAL-L1, MICAL-1,RILP, RILP-L1, } \\
\text { GAPCenA, Leprecan }\end{array}$ \\
\hline Rab 37 & Secretory granules & Exocytosis & RIM1 \\
\hline Rab 38 & Melanosomes & TGN to melanosomes & Varp/Ankrd27 \\
\hline Rab 39 & Golgi & Unknown & Caspase-1 \\
\hline $\begin{array}{l}\text { Rab 40A; Rab 40B; } \\
\text { Rab 40C }\end{array}$ & Golgi, RE & Endosome/Intracellular transport & $\begin{array}{l}\text { Elongin B/C, Cullin5, D-AKAP2, RILP-L1, } \\
\text { RME-8 }\end{array}$ \\
\hline Rab 41 & Golgi & Unknown & Cog6, Golga4/p230, D-AKAP2 \\
\hline Rab 42 & Unknown & Unknown & Unknown \\
\hline Rab 43 & ER, Golgi & ER to Golgi, Shiga toxin transport & Unknown \\
\hline Rab 44 & Unknown & Unknown & Unknown \\
\hline Rab 45 & Perinuclear region & Unknown & Unknown \\
\hline
\end{tabular}

Table A. The RABs family: intracellular location, known pathways, effectors and related diseases.

ER, endoplasmic reticulum; CCVs, clathrin-coated vesicles; TGN, trans-Golgi network; PM, plasma membrane; RE, recycling endosome. Adapted from Reference 165. 


\subsubsection{Uptake, Processing And Clearance Of aSyn}

While it is still unknown why aSyn misfolds and aggregates, several studies performed to address how cells handle aberrant aSyn assemblies.

As already pointed out in the previous sections, aSyn might have a function in synaptic vesicles endocytosis, and the pathological, fibrillary species may use several, different endocytic mechanisms to spread from one cell to another.

There are at least three major endocytic processes to differentiate in synaptic terminals: clathrin-mediated endocytosis ${ }^{183}$ (CME), which occurs in a 10-20 sec range; kiss and run of synaptic vesicles ${ }^{184}$, estimated to occur in less than $1 \mathrm{sec}$ and bulk endocytosis ${ }^{64}$ (Figure 12).

Mechanisms of CME are well established and many contributing elements are known nowadays. CME is mediated by the production of small vesicles with a morphologically characteristic coat composed by the protein clathrin. Clathrin-coated vesicles (CCVs) are found in all the type of cells and can concentrate large extracellular molecules in their domains, playing a role in the endocytosis of several ligands as well ${ }^{185}$.

On the other side, mechanisms responsible of the kiss-and-run endocytosis are still under debate, and is still missing a molecular characterization of the key player involved in the process. However, according to the latest hypothesis, secretory vesicles approaching the plasma membrane do not fully collapse upon membrane fusion: on the contrary, they retain their curvature and release chemical substances through a restricted fusion pore, thus allowing vesicles to preserve their identity during endocytosis $^{186,187}$.

Bulk endocytosis is an endocytic process where a larger area of presynaptic membrane - if compared with the one related to CME - is internalised as cisternae or endosomes: from this newly formed compartment, numerous synaptic vesicles can sprout off. The molecular mechanisms of this endocytic process is still partly unclear. Nevertheless, few important signalling events have been described. Bulk endocytosis can be specifically activated after intense stimulation - as the one obtained with a trains of high-frequency action potentials - or as response to membrane depolarization ${ }^{188}$. In hippocampal neurons CME appears to be the main mechanism ${ }^{183}$, although kissand-run endocytosis has been clearly shown in dopaminergic neurons as well ${ }^{187}$.

Regarding aSyn function in endocytosis, some data are pointing out that aSyn could be associated with CME, as triple aSyn knockout mice show changes in CME-related protein expression and an increase in CCVs after synaptic stimulation ${ }^{189}$. 
A study conducted in lamprey displays alterations of CME and an increase in bulk endocytosis, suggesting that when aSyn related fast endocytosis mechanisms are impaired, a compensatory upregulation of CME can be observed ${ }^{189}$.

On the other hand, a role for aSyn in kiss and run endocytosis can also be possible, considering the ability of this protein on modelling the curvature of small vesicles. Furthermore, the proposed double-anchored mechanisms could influence the tethering of vesicles to the plasma membrane, regulating and preventing the full collapse of synaptic vesicles, as it is required for the above mentioned mechanism ${ }^{64,154}$.

On the same way, every and each of these mechanisms can contribute to the internalization of pathological aSyn from the extracellular space, together with macropinocytosis, passive diffusion throughout the plasma membrane and tunnelling nanotubes.

Due to the use of different pathological assemblies, as well as of different cells and animal models, a unifying theory about the role of aSyn in endocytosis and about the election method for the propagation and spreading of the pathology has not been recognised yet.

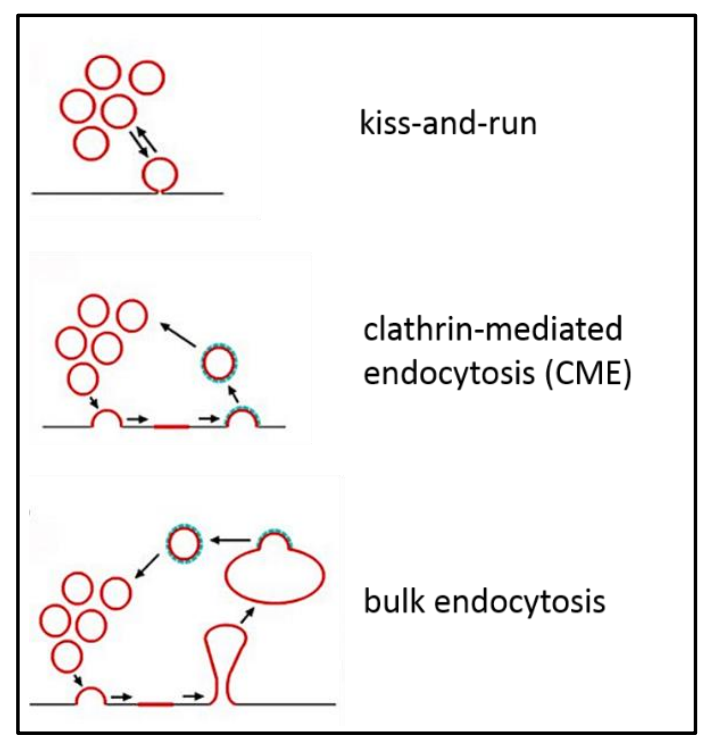

Figure 12. Mechanisms of endocytosis.

Adapted from Http://images.slideplayer.com/17/5285873/slides/slide_24.jpg

Once the pathological aggregates of aSyn enter the cells, a processing mechanism in order to remove those aberrant accumulation starts. Two separate but complementary systems - the Autophagy/Lysosomal pathway (ALP) and the Ubiquitin Proteasome System (UPS), generally handle the clearance of proteins ${ }^{50}$. 
Monomeric, physiological aSyn can be degraded by both system while higher molecular weight species, such as oligomers and fibrils are generally directed to the lysosome for degradation ${ }^{50}$.

Under the name of autophagy are grouped several, different cellular process such as macroautophagy, microautophagy and chaperone-mediated autophagy (CMA) (Figure 13).

CMA is a very complex and specific process based on the recognition of a specific aminoacidic motif (KFERQ) by the cytosolic Hsc70-containing complex ${ }^{50}$. Once formed, the complex interacts with and bind to the lysosome associated membrane protein type 2A (LAMP2A) on the lysosomal membrane, allowing the substrate protein to translocate across the membrane and to enter the lysosomal lumen. Here the protein will be assisted by lysosomal Hsc70 chaperone protein for degradation ${ }^{190,191}$. CMA significantly differs from other types of autophagy because of its selective, oneby-one translocation mechanism of material into the lysosome. While the chaperone mediated is an active process, macroautophagy is a non-selective, constitutively working mechanisms used mainly to eradicate damaged organelles or unused proteins that has undergone through the endosomal pathway ${ }^{50}$.

During macroautophagy, a phagophore engulfs the material that needs to be degraded, trapping this into a double membrane compartment known as an autophagosome ${ }^{192,193}$. The newly formed autophagosome moves through the cytoplasm and eventually fuse together with a lysosome, where its contents is degraded from the acidic lysosomal hydrolases ${ }^{192}$.

On the other hand, the microautophagy process consist of a direct transmigration of cytoplasmic material into the lysosome. This can occurs either through an invagination mechanism or via cellular protrusion ${ }^{194}$.

So far, degradation of aSyn has only been shown to occur through CMA and macroautophagy ${ }^{195}$. Notably, a KFERQ recognition motif was found in the aSyn sequence, suggesting that it can be processed via $\mathrm{CMA}^{194}$. Numerous independent studies revealed that macroautophagy is involved in the degradation of this protein as well, and that aSyn can perturb actively this pathway, as familial mutations, protein overexpression and pathological aggregation have an inhibitory effect on macroautophagy related mechanisms ${ }^{196-198}$.

Besides its involvement in the degradation of misfolded or harmful intracellular components, autophagy play also a major role in the homeostasis of cellular molecules by removing redundant proteins and organelles and by keeping optimal the steadystate level of proteins within the cell. Therefore, it is clear that any failure in this 
quality-control mechanism can affect irreversibly several biological pathways, leading to the accumulation of damaged or abnormally modified proteins and eventually to cell death.

Autophagy is the leading mechanism for aSyn clearance. Under pathological conditions, the cells resort to additional or substitutive mechanisms, such as the release of these pathological assemblies into the extracellular space, thus helping - in an attempt to delay neuronal dysfunction and death - aSyn propagation.

In fact, inhibition of lysosomal function in SH-SY5Y cells that overexpress aSyn led to increased exosome-mediated release of $\mathrm{aSyn}^{199}$, and other studies connected autophagic dysfunction, exocytosis and higher uptake by neighbouring cells via cellto-cell transfer ${ }^{200}$.

In summary, the perturbation of the autophagic process might initiate a cascade of events culminating in apoptotic, dying cells jam-packed with misfolded, aberrant aSyn proteins that will be eventually released in the extracellular space. These aggregates might be internalized by neighbouring cells, acting as seeds for further aggregation and promoting the prion-like spreading of aSyn pathology throughout the brain.

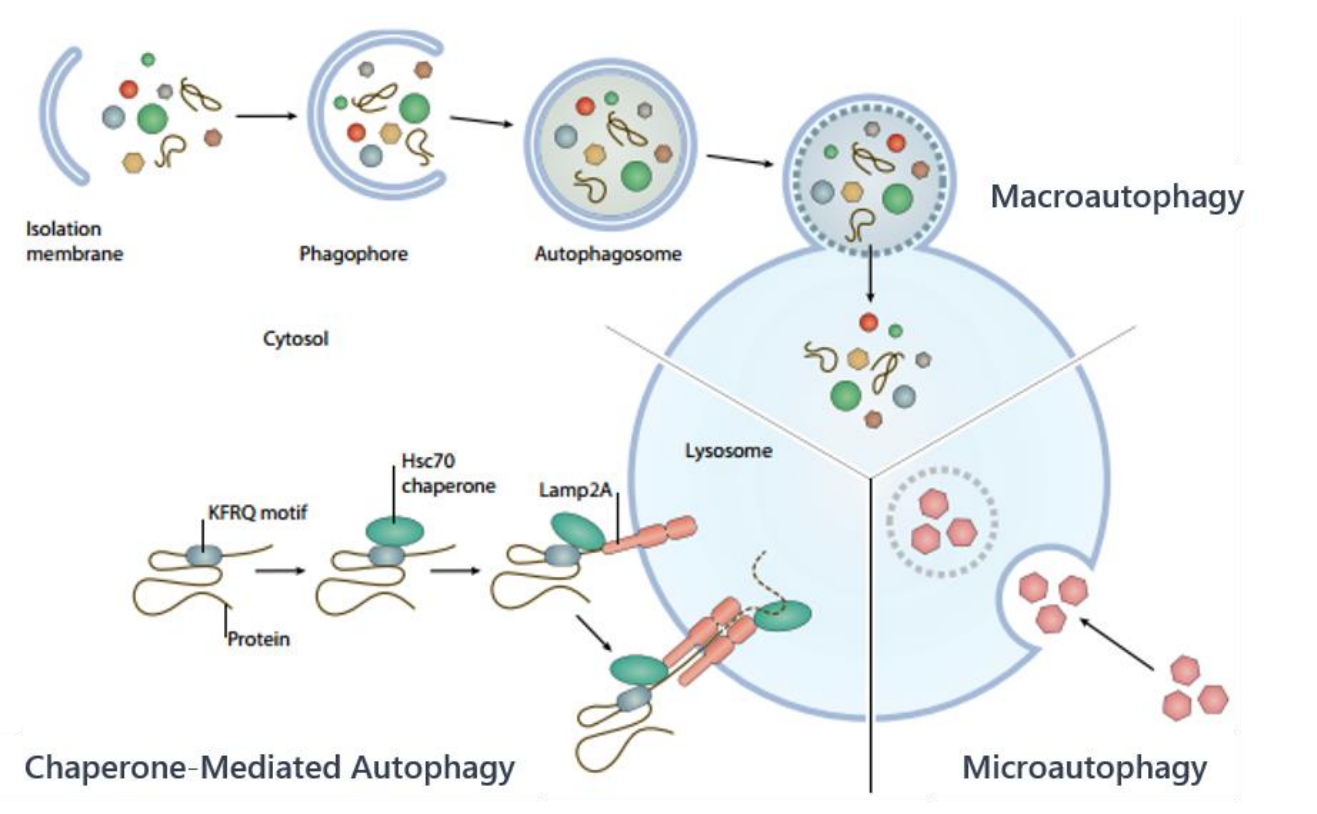

Figure 13. Main type of autophagy in mammalian cells.

In the figure are represented the three main type of autophagy in mammalian cells: macroautophagy, microautophagy, chaperone-mediated autophagy (CMA).

Modified from http://www.antibodycenter.co.kr/bbs/board.php?bo_table=goods\&wr_id=259 


\subsection{Cellular Models For The Study OF ASYN PATHOLOGY}

Developing new therapeutic strategies for treating PD and, more generally, synucleinopathies, requires the use of models.

The use of PD patients is limited by heterogeneity of disease, by the slow evolution of it, by technical difficulties and - last but not least - ethical considerations ${ }^{201}$.

Among the methods and the models available, cultured cells offer numerous advantages for the study of aSyn-related pathology if compared to animal models or directly to humans (patients). Nevertheless, the choice of a specific cell line can reproduce only partially the disease, focusing on one aspect of the pathology while ignoring others. One of the major pro related to the use of cellular models is that these ones - unlike a living organism - develop pathology quickly and reliably, so that researchers can be able to test several methodologies in paralle ${ }^{201}$.

Currently, this "minimalistic" approach allow us to analyse in deep the contribution of specific cellular processes and pathways toward diseases, and in particular, to dissect the mechanisms behind the two most relevant hallmark of PD and other related synucleinopathies : the degeneration of dopaminergic neurons and the formation of aberrant assemblies of aSyn ${ }^{201}$.

Given the relevance of aSyn aggregation in the propagation and the spreading of $\mathrm{PD}$ and synucleinopathies, understanding the driving forces and the mechanisms involved in this process is crucial, and several efforts have been made to address this question ${ }^{202}$. However, unlike other amyloidogenic, aggregation-prone proteins, aSyn does not easily form intracellular inclusions when overexpressed in cultured cells, as the ability of the protein to dimerize and to trigger the formation of high molecular weight species appears to be a rate-limiting step in the aggregates formation process ${ }^{202-204}$. For cell cultures-related work, this constitutes an obstacle, as the experiments need to be completed in a relatively short time, ideally before the dividing cells will overgrow (24 days) or, for neuronal cultures, according to the survival times of primary cultured neurons (1-2 weeks) and it is therefore essential to build up a model that mimic the initial steps of aSyn aggregation ${ }^{201}$.

A powerful tool to study aSyn dimerization and oligomerization is the Bimolecular Fluorescence Complementation assay $(\mathrm{BiFC})^{205,206}$. This method relies on the discoveries that two non-fluorescent fragments of a fluorescent protein can associate to form a fluorescent complex, and that the association of the fragments can be 
facilitated by fusing them to two proteins that interact with each other ${ }^{205}$. In this specific case, the two non-fluorescent fragment are fused to the $\mathrm{N}$-terminal or the C-terminal of aSyn, permitting the visualization of aSyn dimers and oligomers in living cells through the re-constitution of the functioning fluorophore upon aSyn aggregation ${ }^{207}$. On the other side, manipulations of the C-terminal region of aSyn have proven very effective to investigate the formation of mature aggregates and the development of LBlike inclusions.

Using a series of aSyn enhanced green fluorescent protein (EGFP)-fusion proteins as a tool for studying aSyn aggregation, it was found that, when transfected in cultured cells, the $49 \mathrm{kDa}$ aSyn-EGFP fusion protein is partially truncated to a $27 \mathrm{kDa}$ form, and that this non-fluorescent carboxy-terminally modified protein, named SynT, spontaneously forms inclusions in the neuronal cytoplasm ${ }^{208}$ (Figure 14). Aggregation of SynT was further enhanced by co-transfection of Synphilin-1, an interactor partner of aSyn present in LBs and LNs. Given the presynaptic location and its affinity for membranes and lipids ${ }^{209}$, Synphilin-1 also seems to be required for inclusion formation ${ }^{210}$ and might act as an adaptor protein, supporting the interaction of aSyn with other molecules ${ }^{211}$. Co-transfection of SynT with Synphilin-1 further increase the propensity for aggregation of aSyn, by rising the frequency and the size of inclusions and leading to the formation of large, ring-shaped aggregates that mimic the halo structure of $\mathrm{LB}^{212}$ and that show Thioflavine S (ThioS) immunoreactivity ${ }^{213,214}$. While powerful and versatile, these models rely on the addition of extraneous amino acid sequences to aSyn, which alter its biochemical and biophysical properties. 


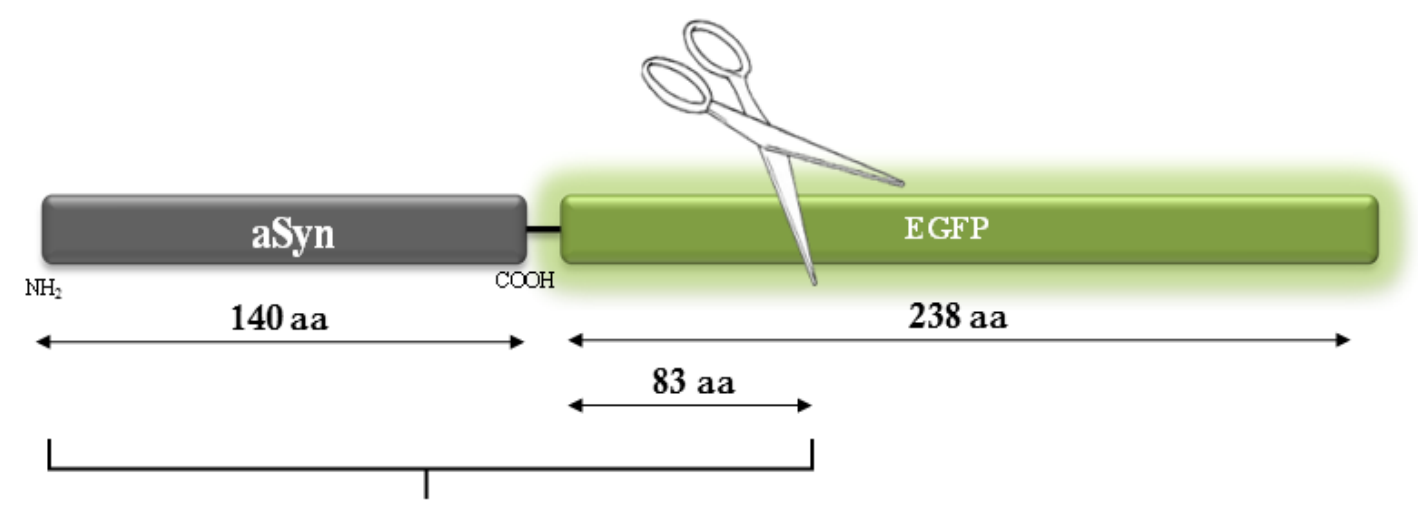

SynT

234 aa

Figure 14. Schematic representation of SynT .

The overexpression of SynT in cultured cells allow us to investigate the processes related to the formation of insoluble aggregates. SynT is a protein of ca $27 \mathrm{kDa}$ resulting from the fusion of aSyn WT with a truncated, not-fluorescent fragment of EGFP. 


$$
\begin{gathered}
-I I- \\
\text { Aims OF THE STUdy }
\end{gathered}
$$




\section{Aims Of The Study}

The first aim of the study was to analyse the mechanisms of interaction of aSyn with membranes and trafficking machinery pathways.

Our goals were to:

- Determine whether different species of aSyn (monomers and fibrils) can enter cultured cells and form inclusions.

- Investigate how different species of aSyn can interact with cellular membranes and with the trafficking machinery pathways involved in the internalization and clearance of aSyn through the use of mutated recombinant proteins (aSyn WT, aSyn A30P and aSyn A11P/V70P) and the screening of RAB-GTPases family proteins.

The second aim of the project was to characterize the biophysical, structural properties of an aggregation-prone variant of aSyn, SynT, able to mimic the pathological behaviour of the protein. In this context, we analysed the structural effects of tagging aSyn at the C-terminus (SynT), its aggregation propensity and its conformational properties by using an in vitro, NMR-based approach. 


$$
\text { - III - }
$$

MATERIALS AND

METHODS 


\subsection{PURIFICATION AND CHARACTERIZATION OF RECOMBINANT PROTEINS}

\subsubsection{Protein Purification}

aSyn WT, aSyn A30P and aSyn A11P/V70P were obtained by transforming E.coli BL21-DE3 ultracompetent cells with plasmids encoding corresponding cDNA sequences (pET21-aSyn, pET21-A30P, pET21-A11P/V70P).

${ }^{15} \mathrm{~N}$ isotopically enriched and $\mathrm{N}$-terminally acetylated aSyn and SynT were obtained by transforming E.coli BL21 ultracompetent cells with a plasmid carrying the aSyn WT gene or the SynT gene together with a second plasmid encoding the components of yeast $\mathrm{NatB}$ acetylase complex ${ }^{215}$. Plasmids carried different antibiotic resistance, in this case for ampicillin and chloramphenicol, to select $E$. coli colonies transformed with both the plasmids.

Purification was performed as previously reported ${ }^{36}$ with some minor modifications. Briefly, BL21-DE3 competent cells were grown in LB medium (or in M9 medium enriched with $\mathrm{NH}_{4} \mathrm{Cl}$ when necessary) in the presence of ampicillin $(100 \mu \mathrm{g} / \mathrm{ml})$ or in the presence of ampicillin $(100 \mu \mathrm{g} / \mathrm{ml})$ and chloramphenicol $(1 \mathrm{mg} / \mathrm{ml})$.

Protein expression was induced with $1 \mathrm{mM}$ of isopropyl-1-thio- $\beta$-D-galactopyranoside (IPTG) for $4 \mathrm{~h}$ at $37^{\circ} \mathrm{C}$. The cultures were then centrifuged (30 min at 4.500 to $6.000 \mathrm{xg}$ ) and the cell pellet was resuspended in Lysis Buffer ( $50 \mathrm{mM}$ Tris HCL, $150 \mathrm{mM} \mathrm{NaCl}$, $1 \mathrm{mM}$ EDTA and Inhibitor Protease cocktail $\alpha$-complete (La Roche, Basel, Switzerland)) at $\mathrm{pH} 8.0$.

Cells were recovered, sonicated on ice, boiled for $20 \mathrm{~min}$ at $95^{\circ} \mathrm{C}$, and cell debris were discarded by centrifugation. Subsequent precipitation first with streptomycin sulphate $(10 \mathrm{mg} / \mathrm{ml})$ and later with ammonium sulphate $(361 \mathrm{mg} / \mathrm{ml})$ was performed in order to obtain a precipitate enriched with aSyn.

Anion exchange high-performance liquid-chromatography (AEC) was carried out on an Äkta-HPLC Purifier (GE Healthcare).

The pellet was resuspended then in $25 \mathrm{mM}$ Tris- $\mathrm{HCl}(\mathrm{pH}$ 7.7), and loaded onto a Mono Q column on an Applied Biosystems BIOCAD (Applied Biosystems, Foster City, CA) or bounded to an Hi-Trap column (GE Healthcare).

The monomeric proteins were eluted at $\sim 300 \mathrm{mM} \mathrm{NaCl}$ with a linear gradient of elution buffer from $0 \mathrm{mM}$ to $1 \mathrm{M} \mathrm{NaCl}$. The pure proteins (judged by PAGE) were 
dialyzed overnight against the appropriate buffer and further size exclusion chromatography (SEC) purification step using a Superdex 75 column was performed. Protein concentration was estimated from the absorbance at $274 \mathrm{~nm}$ using an extinction coefficient of $5600 \mathrm{M}^{-1} \mathrm{~cm}^{-1}$ for aSyn WT, aSyn A30P, aSyn A11P/V70P and of $16055 \mathrm{M}^{-1} \mathrm{~cm}^{-1}$ for SynT.

Protein samples were dissolved in buffer A (25 mM Tris, $\mathrm{pH} 7.7)$ or buffer B (20 mM MES, $\mathrm{NaCl} 100 \mathrm{mM}, \mathrm{pH}$ 6.5). The protein stocks were filtered through $0.22 \mu \mathrm{m}$ filter unit and then frozen in aliquots at $-80^{\circ} \mathrm{C}$.

\subsubsection{FIBRIL FORMATION}

The integrity and stability of aSyn monomers, besides the lack of soluble oligomers at the first steps of the reaction was controlled through a gel filtration chromatography using a Superdex 75 10/300 column (GE Healthcare Life Sciences). Aliquots of aSyn WT were prepared from the protein stocks in triplicates of $0.3 \mathrm{~mL}$ each. The samples were then diluted or in phosphate saline buffer (PBS), or in Buffer B for a concentration of $60 \mu \mathrm{M}$. To the resulting samples it was then added sodium azide at a final concentration of $0.02 \%$, followed by incubation at $37^{\circ} \mathrm{C}, 600 \mathrm{rpm}$ in a mixer with temperature control (Eppendorf Thermomixer Comfort, Eppendorf, USA).

The formation of aSyn aggregates was monitored by measuring light scattering with a spectrofluorometer (Jasco Inc, MD, USA) with parameters of excitation/emission adjusted for 330/320-340 nm. For fluorometric measurements, all samples were diluted in native buffer for a concentration of $10 \mu \mathrm{M}$. As a negative control solutions without protein were used.

\subsubsection{THIOFLAVINE T AsSAYS}

In vitro aggregation kinetics was obtained using a PolarStar OMEGA microplate reader. The plate was shaken at $300 \mathrm{rpm} / 37^{\circ} \mathrm{C}$ and fibril formation was monitored by measuring Thioflavine $\mathrm{T}$ (Thio- $\mathrm{T}$ ) fluorescence every $5 \mathrm{~min}$. The excitation wavelength was set to $440 \mathrm{~nm}$ and the Thio-T emission was measured at $480 \mathrm{~nm}$. Each well contained $150 \mu \mathrm{L}$ of $25 \mu \mathrm{M}$ aSyn or $25 \mu \mathrm{M}$ SynT (Buffer A), $1 \mu \mathrm{M}$ Thio-T, and a $2 \mathrm{~mm}$ glass bead to accelerate fibrillation. In all cases, the reported values correspond to the average of five independent aggregation measurements. 


\subsubsection{TRASMISSION ELECTRON MicROSCOPY}

To test the effective presence of fibrillar aggregates, samples of aSyn or SynT were plated onto carbon-coated grids, treated and stained with $2 \%(\mathrm{w} / \mathrm{v})$ uranyl acetate. After the end of the treatment, samples were analysed and representative fields were imaged in a Hitachi H-7000 Transmission Electron Microscopy (TEM) operating with a $75 \mathrm{kV}$ voltage.

\subsection{Cell Culture And Molecular Biology TECHNIQUES}

\subsubsection{Human Cell Culture}

Human neuroglioma $\mathrm{H} 4$ cells were maintained at $37^{\circ} \mathrm{C}$ and $5 \% \mathrm{CO}_{2}$ environment, in Opti-Mem medium (PAN, Germany) supplemented with $10 \%$ foetal bovine serum (FBS) (ThermoFisher) and 1\% penicillin-streptomycin (ThermoFisher). For the in vitro experiments related to the internalization of aSyn monomers and fibrils, cells were routinely seeded $24 \mathrm{~h}$ prior to use in different well-plate formats, at a density of 8.5 to $10^{*} 10^{4}$ cells $/ \mathrm{ml}$. Cell count was determined using a Neubauer counting chamber. The day after, cells were treated with different concentrations of aSyn monomers or aSyn fibrils for $24 \mathrm{~h}$. After the end of the treatment, cells were extensively washed with PBS (3 times, $5 \mathrm{~min}$ ), therefore briefly treated with trypsin in order to remove the residual proteins still attached to the dish (for a maximum time of 30 seconds), incubated again with medium (in order to stop the trypsinization related reactions) and then washed one last time with PBS.

At this point, it was possible to proceed with the fixation with paraformaldehyde (PFA) for the Immunocytochemistry (ICC) or with the cell lysis and protein extraction for the immunoblotting analysis. 


\subsubsection{Transient Transfection Of Cultured Cells}

For the Rab-GTPase family screening experiments, as well as for the SynT-Synphilin1 aggregation model, cells were seeded in different well-plate formats, one day before the transfection, and treated during $48 \mathrm{~h}$ after transfection.

Transfections were performed with calcium phosphate method (CaPho method) (www.flemingtonlab.com). Shortly, 3h before the transfection it was added to the cultured cell fresh medium. DNA was diluted in 1x HBS buffer with $25 \mathrm{mM}$ 4-(2hydroxyethyl)-1-piperazineethanesulfonic acid, $140 \mathrm{mM} \mathrm{NaCl}, 5 \mathrm{mM} \mathrm{KCl}, 0.75 \mathrm{mM}$ $\mathrm{Na}_{2} \mathrm{HPO}_{4} \cdot 2 \mathrm{H}_{2} \mathrm{O}, 6 \mathrm{mM}$ Dextrose, $\mathrm{pH}$ 7.1. Later on, $2.5 \mathrm{M} \mathrm{CaCl}_{2}$ was added dropwise and mixed immediately. After 20 min of incubation, the mixture obtained was added to the cells. The next morning cells were fed again with fresh medium, and then incubated with aSyn monomers or fibrils as described previously.

\subsubsection{Solubilisation Of Proteins And Protein QUANTIFICATION}

Cells were solubilized $48 \mathrm{~h}$ after transfection step (unless indicated otherwise) using radioimmuniprecipitation assay (RIPA) buffer (50 mM Tris $\mathrm{pH} 8.0,150 \mathrm{mM} \mathrm{NaCl}$, $0.1 \%$ Sodium-Dodecyl-Sulphate (SDS), $\quad 1 \%$ Nonidet P40, $0.5 \%$ SodiumDeoxycholate, $\alpha$-complete (La Roche, Basel, Switzerland). The resulting cell lysates were then centrifuged for $15 \mathrm{~min}$ at maximum speed (Sigma 1-15 K, Sigma Aldrich Co. LLC, St. Louis, MO, USA). Following centrifugation, the supernatant was then transferred into a new microtube. Protein concentration was determined through Bradford's assay. Briefly, $1 \mu 1$ of the cell lysate was diluted with $49 \mu 1$ of sterile water and $150 \mu \mathrm{l}$ of Bio-Rad Protein Assay Dye Reagent Concentrate (Bio-Rad Laboratories, Inc., Hercules, CA, USA). Absorbance (595 nm/450 nm) was measured using the Infinite M200 PRO (Tecan Ltd., Maennedorf, Switzerland) plate reader. For all the experiments performed, samples were measured in triplicate. 


\subsubsection{Western Blot ANALyses}

Detection of proteins was performed with immunoblot analysis to detect aSyn, V5, $\beta$ actin, tubulin, transferrin receptor and Rab GTPases fused to GFP.

Cell lysates were separated by sodium dodecylsulphate polyacrylamide gel electrophoresis (SDS-PAGE) using reducing conditions, such as 12\% separating gels and 7\% stacking gels (Acrylamide 37.5 (Carl Roth $\mathrm{GmbH}$, Karlsruhe, Germany), 0.01 $\%$ SDS in ddH2O, $0.1 \%$ tetramethylethylenediamine (TEMED), and $1 \%$ ammonium persulfate (APS).

A total amount of $40 \mu \mathrm{g}$ of sample for each of the lysates was loaded with $5 \mathrm{x}$ Laemmli buffer $(250 \mathrm{mM}$ Tris $\mathrm{pH}$ 6.8, 10\% SDS, 1.25\% Bromophenol Blue, 5\% $\beta$ Mercaptoethanol, $50 \%$ Glycerol) and boiled for $5 \mathrm{~min}$ at $95^{\circ} \mathrm{C}$.

the addition of $5 \mu$ l PageRuler Plus Protein Ladder (Perbio Science Deutschland, Bonn, Germany), samples were loaded and run in the gel.

Electrophoresis experiment was performed with the use of SDS-Running Buffer (125 $\mathrm{mM}$ Tris, $960 \mathrm{mM}$ Glycine) for $90 \mathrm{~min}$ at $100 \mathrm{~V}$ in Bio-Rad Mini-Protean 3 Mini Vertical Electrophoresis System (Bio-Rad Laboratories, Inc., Hercules, CA, USA).

Following electrophoresis step, proteins were transferred in a $0.45 \mu \mathrm{m}$ nitrocellulose membranes using a semi-dry transfer chamber Trans-Blot ${ }^{\circledR}$ Turbo ${ }^{\mathrm{TM}}$ Transfer Solution from Bio-Rad (Bio-Rad Laboratories, Inc., Hercules, CA, USA). Samples run for 30 min in the chamber with a constant current adjusted for $25 \mathrm{~mA}$.

In other to block free sites, membranes were incubated with $10 \%(\mathrm{w} / \mathrm{v})$ skim milk dissolved in TRIS buffered saline supplemented with Tween-20 (TBS-T) (50 mM Tris (hydroxymethyl)-aminomethane (TRIS) supplemented with $0.05 \%$ (v/v) Tween-20) for $1 \mathrm{~h}$ at Room Temperature (RT). For detection of the specific proteins, the primary antibodies were dissolved in TBS and incubated on a shaker overnight at $4^{\circ} \mathrm{C}$. Afterwards, membranes were washed three times (for 10 minutes each) with TBS-T and then incubated with secondary antibodies HRP-conjugated (diluted 1:10000 in TBS-T) for $2 \mathrm{~h}$ at RT. Membranes were visualized using Fusion Fx (Vilber Lourmat, Marne-la-Vallée, France) with Immobilon Western Chemiluminescent HRP Substrate (Merck Millipore, Billerica, MA, USA).

\subsubsection{Dot Blot Analyses}

All HPLC samples were incubated for $10 \mathrm{~min}$ at $95^{\circ} \mathrm{C}$, pulled down at $10,000 \mathrm{xg}$ and $4^{\circ} \mathrm{C}$, and then loaded in a 96 well homemade Dot Blot machine. 
Before loading them, the samples were filtered through a $0.2 \mu \mathrm{m}$ pore size Protean nitrocellulose membrane (Schleicher \& Schuell Bioscience GmbH, Dassel, Germany) with the use of a vacuum pump. The membrane was then incubated along $1 \mathrm{~h}$ with blocking buffer containing 5\% skim milk in TBS to avoid unspecific staining. Later, membranes were exposed to primary antibody diluted in $1 \%$ skim milk in TBS or $5 \%$ bovine serum albumin (BSA) in TBS (aSyn BD transduction, 1:2000) overnight at $4^{\circ} \mathrm{C}$. After being washed three times with TBS-T for $10 \mathrm{~min}$ each, membranes were incubated with HRP-conjugated secondary antibody diluted 1:10,000 in TBS. Lastly, protein bands were detected using a chemiluminescent substrate (Merck Millipore, Billerica, MA, USA), and membranes were imaged in a Fusion Fx photo documentation system (Vilber Lourmat, Marne-la-Vallée, France).

\subsubsection{TRITON X-100 FRACTIONATION ASSAY}

H4 cells were plated and treated (with monomers or fibrils of recombinant aSyn) as described above. At the end of the treatment, cells were lysed in Lysis Buffer I ( $25 \mathrm{mM}$ Tris $\mathrm{pH} 7.5,150 \mathrm{mM} \mathrm{NaCl}, 1 \mathrm{mM}$ EDTA and cocktail of protease inhibitors) and centrifuged at $100.000 \mathrm{~g}$ for 30 minutes at $4 \mathrm{C}$. Supernatants were collected (soluble fraction) and the pellets (insoluble fraction) were washed with cold PBS and transferred to new tubes.

Samples were centrifuged once again $14.000 \mathrm{rpm}$ for 10 minutes at $4^{\circ} \mathrm{C}$ and the resulting pellet, corresponding to the insoluble fraction, was subsequently resuspended in Lysis Buffer II (75 mM Tris, pH 6.8, 3\% SDS, 15\% Glycerin, 3.75 mM EDTA pH 7.4 and a cocktail of protease inhibitors).

Finally, samples were sonicated (10 pulse/second) and immunoblotting analysis were performed as described above.

\subsubsection{BiotinyLATION AsSAY}

The day before the experiment, cells were plated in $100 \mathrm{~mm}$ Petri Dishes at a density of $4^{*} 10^{6}$ cells, and grown until $60-70 \%$ confluence. Thereafter, cells were treated with $1 \mu \mathrm{M}$ of aSyn recombinant monomers or fibrils of different aSyn variants (WT, aSyn A30P or aSyn A11P/V70P) for $24 \mathrm{~h}$. 
At the end of the treatment, cells were rinsed 3 times in ice-cold PBS and further incubated in PBS containing $1.5 \mathrm{mg} / \mathrm{ml}$ of EZ-Link Sulfo-NHS-SS-Biotin (ThermoFisher) with gentle rocking, for $30 \mathrm{~min}$, at $4^{\circ} \mathrm{C}$. The non-bound biotin was removed by incubating cells with $100 \mathrm{mM}$ solution of Glycine for $15 \mathrm{~min}$ at $4^{\circ} \mathrm{C}$.

In order to remove the excess of Glycine, cells were briefly washed with PBS and thereafter cell lysate was prepared in PBS containing Protease Inhibitor $\alpha$-complete (La Roche, Basel, Switzerland), 0.1\% SDS and 1\% Triton X-100. The lysates were sonicated for $30 \mathrm{sec}$ and centrifuged for $5 \mathrm{~min}$ at $17000 \mathrm{x} \mathrm{g}$.

The supernatant was further incubated with $100 \mu \mathrm{L}$ of NeutrAvidin Agarose Resin (ThermoFisher) for $2 \mathrm{~h}$, in a rotatory shaker with gentle agitation, at $4^{\circ} \mathrm{C}$.

After the incubation with the resin, the supernatant (corresponding to the Cytoplasmic cell lysate fraction) was collected, and a Bradford assay was performed to evaluate the amount of total protein concentration in each of the samples.

Biotinylated proteins were then washed 3 times with PBS and then eluted with $2 x$ Sample Loading Bugger (Laemmli Buffer) by boiling the samples at $95^{\circ} \mathrm{C}$ for $5 \mathrm{~min}$.

Samples were then processed by western blotting. Transferrin receptor was used as a positive control of the biotinylated fraction, whereas tubulin was used as a positive control for the cytoplasmic cell lysate fraction.

This protocol and the many used of biotinylation products were extensively described previously ${ }^{216,217}$. A schematic of the process is shown in Figure 15.

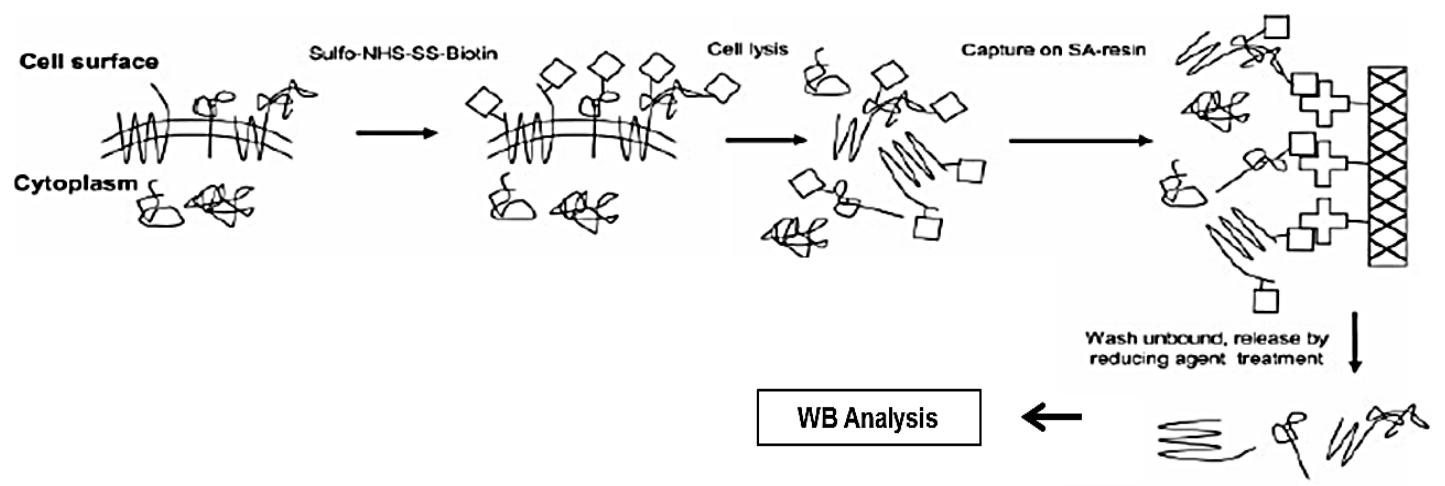

Figure 15. Schematic of the biotinylation assay.

Cell surface proteins are covalently modified with sulfo-NHS-SS-biotin, a biotin derivative carrying a cleavable linker and reactive toward primary amino groups. After cell lysis in the presence of detergents, biotin-labeled proteins are purified on SA-coated resin. Following their elution, isolated proteins are separated and analyzed via immunoblot analysis. Modified from Reference 217. 


\subsection{IMMUNOFLUORESCENCE, MICROSCOPY AND IMAGING}

\subsubsection{IMMUNOCYTOCHEMISTRY}

For ICC analysis, cells were plated in multi-well plates with different formats, previously coated with coverslips.

For the in vitro experiments related to the internalization of aSyn monomers and fibrils, for the RABs screening experiments and for the SynT-Synphilin-1 aggregation model cells were routinely seeded $24 \mathrm{~h}$ prior to use in different well-plate formats, at a density of $8.5^{*} 10^{4}$ cells $/ \mathrm{ml}$. Cell count was determined using a Neubauer counting chamber. The day after, cells were transfected when necessary and then treated with different concentrations of aSyn monomers or aSyn fibrils for $24 \mathrm{~h}$

24 or $48 \mathrm{~h}$ after transfection, H4 cells were washed with PBS and fixed with 4\% PFA supplemented with $0.4 \%$ Sucrose for 10 minutes at RT. A permeabilisation step with 0.5\% Triton X-100 (Sigma Aldrich, St. Louis, MO, USA) for 10 minutes at RT was then followed by the blocking process, performed with $10 \%$ normal goat serum (PAA, Cölbe, Germany)/DPBS for $1 \mathrm{~h}$.

After the blocking step, cells were incubated with primary antibodies overnight.

After washing 3 times for 10 min with PBS to remove the unbound primary antibody, samples were incubated with secondary antibodies for $2 \mathrm{~h}$ at RT.

In some of the experiments cells were incubated with Phalloidin (Phalloidin 488 or Phalloidin 594), in order to stain acting filaments. Phalloidin (1:50 in PBS) was added to the samples after the secondary antibody, for 1-2 hours at RT.

Finally, cells were washed again 3 times for 10 minutes with PBS and therefore stained with Hoechst 33258 (Life Technologies- Invitrogen, Carlsbad, CA,USA) (1:5000 in DPBS) for 5 minutes, washed again with PBS and then fixed with Mowiol for epifluorescence microscopy.

All the primary and secondary Antibodies used are reported in Table B. 


\subsubsection{MicROSCOPY AND IMAGING}

Images in Figure 16 and in Figure 30 were acquired using a Leica Inverted Microscope DMI 6000 B (Leica, Wetzlar, Germany), using a 40x objective (HCX P1 Fluotar) or a 63x objective (HCX P1 Fluotar).

For the screening of RAB proteins, images were taken using an Olympus IX81-ZDC microscope system, with a $40 \times$ objective (FN 26,5). 64 images were randomly taken using the Olympus $\mathrm{Scan}^{\wedge} \mathrm{R}$ Image Analysis Software out of three independent experiments. All the other images were acquired using a Confocal Zeiss LSM800 with $40 \mathrm{x}$ or $63 \mathrm{x}$ oil immersion objectives.

For the colocalization analysis in Figure 20, the Pearson's $\mathrm{R}$ value was calculated via the use of Coloc2 plugin from ImageJ.

For the mean fluorescence intensity values in Figures 26, 27, 28 and 29 was used Fiji (ImageJ) Software and GraphPad Prism for the statistical analyses and graph generation. 


\begin{tabular}{|c|c|c|c|c|}
\hline \multicolumn{5}{|c|}{ Primary Antibodies } \\
\hline Antibody & Species & Manufacturer & N. catalog & Dilution \\
\hline Human aSyn c-20 & rabbit & $\begin{array}{l}\text { Santa Cruz } \\
\text { Biotechnology }\end{array}$ & sc-7011-R & $1: 1000 / 1: 2000$ \\
\hline Human aSyn & mouse & BD transduction & 610787 & $1: 2000$ \\
\hline Actin beta & mouse & Sigma-Aldrich & A5441 & $1: 10000$ \\
\hline Tubuline b III & mouse & $\begin{array}{l}\text { Santa Cruz } \\
\text { Biotechnology }\end{array}$ & sc-80005 & $1: 5000 / 10000$ \\
\hline Transferrin receptor & mouse & $\begin{array}{l}\text { Invitrogen / Novex } \\
\text { Life Technologies }\end{array}$ & $13-6890$ & $1: 1000$ \\
\hline v5 & mouse & $\begin{array}{l}\text { Invitrogen/Novex } \\
\text { Life Technologies }\end{array}$ & 46-0705 & $1: 1000$ \\
\hline GFP & mouse & Roche & $1,1814 \mathrm{E}+10$ & $1: 1000$ \\
\hline
\end{tabular}

\begin{tabular}{|c|c|c|c|c|}
\hline \multicolumn{5}{|c|}{ Secondary Antibodies } \\
\hline Antibody & Species & Manufacturer & N. catalog & Dilution \\
\hline $\begin{array}{l}\text { ECL Mouse IgG, HRP- } \\
\text { linked }\end{array}$ & mouse & GE Healthcare & NXA931 & $1: 10.000$ \\
\hline $\begin{array}{l}\text { ECL Rabbit igG, HRP- } \\
\text { linked }\end{array}$ & rabbit & GE Healthcare & NA934V & $1: 10.000$ \\
\hline $\begin{array}{l}\text { Alexa Fluor } 488 \\
\text { donkey anti mouse }\end{array}$ & mouse & $\begin{array}{l}\text { Thermo Fisher } \\
\text { Scientific }\end{array}$ & A21202 & $1: 1000$ \\
\hline $\begin{array}{l}\text { Alexa Fluor } 488 \\
\text { donkey anti rabbit }\end{array}$ & rabbit & $\begin{array}{l}\text { Thermo Fisher } \\
\text { Scientific }\end{array}$ & A21206 & $1: 1000$ \\
\hline $\begin{array}{l}\text { Alexa Fluor } 555 \\
\text { donkey anti mouse }\end{array}$ & mouse & $\begin{array}{l}\text { Thermo Fisher } \\
\text { Scientific }\end{array}$ & A31570 & $1: 1000$ \\
\hline $\begin{array}{l}\text { Alexa Fluor } 555 \\
\text { donkey anti rabbit }\end{array}$ & rabbit & $\begin{array}{l}\text { Thermo Fisher } \\
\text { Scientific }\end{array}$ & A31572 & $1: 1000$ \\
\hline DAPI & - & Roth & 6335.1 & $1: 5000$ \\
\hline Phalloidin-594 & - & $\begin{array}{l}\text { Thermo Fisher } \\
\text { Scientific }\end{array}$ & A12381 & $1: 200$ \\
\hline Phalloidin - 488 & - & $\begin{array}{l}\text { Thermo Fisher } \\
\text { Scientific }\end{array}$ & A12379 & $1: 200$ \\
\hline
\end{tabular}

Table B. List of primarv and secondarv Antibodies used. 


\subsection{STRUCTURAL BiOLOGY}

\subsubsection{Circular Dichroism ANALYSIS}

Circular Dichroism (CD) spectra were recorded at $25^{\circ} \mathrm{C}$ on a JASCO J-1500 spectropolarimeter. aSyn and SynT samples were diluted in Buffer A at a concentration of $20 \mu \mathrm{M}$ and loaded into $1 \mathrm{~mm}$ path-length cuvettes. We averaged 8 scans for each sample to increase signal-to-noise ratios.

\subsubsection{SUVs PREPARATION}

SUVs were prepared from a molar ratio of 1:1 of Coagulation Reagent I containing DOPE:DOPS:DOPC (5:3:2 w/w) and DOPC (both Avanti Polar Lipids Inc., USA) dissolved in chloroform yielding a final molar ratio of DOPE:DOPS:DOPC (5:3:12 $\mathrm{w} / \mathrm{w})$. The lipid solution formed a thin film under evaporation of the solvent with nitrogen gas and was further dried by lyophilisation under vacuum. The dried phospholipids were dissolved in MES buffer (20 mM MES, $100 \mathrm{mM} \mathrm{NaCl}, \mathrm{pH}$ 6.5) and underwent several cycles of freeze-thawing and water bath sonication until the solution became clear. The size distribution was also checked by DLS. For the NMR experiments a SUV stock solution of $85 \mathrm{mM}(6.6 \% \mathrm{w} / \mathrm{v})$ in respect to the monomers was used.

\subsubsection{Nuclear Magnetic Resonance}

All NMR spectra were recorded on a Bruker $600 \mathrm{MHz}$ Advance III spectrometer, equipped with a cryogenically cooled triple resonance ${ }^{1} \mathrm{H}\left({ }^{13} \mathrm{C} /{ }^{15} \mathrm{~N}\right) \mathrm{TCI}$ probe. ${ }^{1} \mathrm{H}-{ }^{15} \mathrm{~N}$ SOFAST-HMQC ${ }^{218},{ }^{1} \mathrm{H}_{-}{ }^{15} \mathrm{~N}$ HSQC, ${ }^{15} \mathrm{~N}$ R1 and ${ }^{15} \mathrm{~N}$ R2 relaxation rates, ${ }^{1} \mathrm{H}_{-}-{ }^{15} \mathrm{~N}$ hetNOE and J-coupling HNHA experiments were all recorded at $15^{\circ} \mathrm{C}$ using protein samples dissolved in Buffer B supplemented with $10 \% \mathrm{D}_{2} \mathrm{O}$. NMR parameters used in each experiment are described next.

${ }^{1} \mathrm{H}-{ }^{15} \mathrm{~N}$ SOFAST-HMQC and ${ }^{1} \mathrm{H}-{ }^{15} \mathrm{~N}$ HSQC: we used 16 scans, 1024 complex points (sweep-width of $16 \mathrm{ppm}$ in the ${ }^{1} \mathrm{H}$ dimension) and 256 complex points (sweep-width 
of $26 \mathrm{ppm}$ in the ${ }^{15} \mathrm{~N}$ dimension). Sequence-specific assignments for the backbone of aSyn and SynT were transferred from previously published work $^{219,106}$. Only unambiguously assigned, well resolved peaks were included in the analysis. The $\mathrm{I} / \mathrm{I}_{0}$ ratios obtained for aSyn and SynT, in the absence or in the presence of PcTS or SUVs were plotted as a function of the protein sequence to obtain the intensity perturbation profiles ${ }^{220}$. Mean weighted chemical shifts displacements (MW $\Delta C S$ ) for ${ }^{1} \mathrm{H}^{-15} \mathrm{~N}$ were calculated as $\left[\left(\Delta \delta^{1} \mathrm{H}\right)^{2}+\left(\Delta \delta^{15} \mathrm{~N} / 10\right)^{2}\right]^{1 / 2}$.

${ }^{15} \mathrm{~N} \mathrm{R}_{1}$ and $\mathrm{R}_{2}$ relaxation rates, and ${ }^{1} \mathrm{H}^{-15} \mathrm{~N}$ NOE data were acquired at $600 \mathrm{MHz}$ external field using previously described pulse sequences ${ }^{221}$. Experiments were recorded with 1024 complex points and a sweep width of $16 \mathrm{ppm}$ for the ${ }^{1} \mathrm{H}$ dimension, and 256 complex points in the ${ }^{15} \mathrm{~N}$ dimension with a sweep width of $26 \mathrm{ppm} . \mathrm{R}_{1}$ and $R_{2}$ relaxation rates were obtained by recording the experiments with different $T_{1}$ and $\mathrm{T}_{2}$ relaxation delays. Resonance heights in the spectra at each delay were fit to a two parameter exponential decay function to obtain the rates. Steady-state ${ }^{1} \mathrm{H}^{-15} \mathrm{~N}$ NOE (hetNOEs) values were obtained from the ratio of peak heights in spectra collected with and without an initial $4 \mathrm{sec}$ period of proton saturation during the recycling delay. HNHA experiments were recorded with the following set up: number of points, 1024 $\left({ }^{1} \mathrm{H}\right), 80\left({ }^{15} \mathrm{~N}\right), 144\left({ }^{1} \mathrm{H}\right)$; spectral width (ppm), $16\left({ }^{1} \mathrm{H}\right), 26\left({ }^{15} \mathrm{~N}\right), 10(1 \mathrm{H})$; number of scans, 16. Three-bound $\mathrm{HN}-\mathrm{H} \alpha$ coupling constants ( $\left.{ }^{3} \mathrm{~J} \mathrm{HN}-\mathrm{H} \alpha\right)$ were obtained from the ratio between the intensities of the diagonal peaks and cross-peaks in the HN-HA correlation region ${ }^{219,222}$. The aforementioned coupling constants are sensitive to the torsion angle $\phi$ populated by each amide group in the protein backbone, and are reflecting on the secondary structure content of the protein analysed ${ }^{219}$. This coupling falls in the range $3.0-6.0 \mathrm{~Hz}$ for $\alpha$-helix and $8.0-11.0 \mathrm{~Hz}$ for a $\beta$-sheet structures. For a random-coil, the weighted average of the values observed ranges typically between 6.0 and $8.0 \mathrm{~Hz}$ for most residues ${ }^{223}$.

Acquisition and processing of NMR spectra were performed using TOPSPIN 3.2 (Bruker Biospin). 2D spectra analyses were performed with CCPN. For the ${ }^{3} \mathrm{~J} \mathrm{HN}-\mathrm{H} \alpha$ couplings calculation, the software CARA was used. $\mathrm{R}_{1}$ and $\mathrm{R}_{2}$ relaxation data fitting was performed using CCPN routines. 


\subsection{SOFTWARE AND STATISTCS}

Statistical analysis was performed using Microsoft Excel (Microsoft Corporation) and GraphPad PRISM 5 (GraphPad Software, San Diego, CA, USA). Images were processed with ImageJ V1.41, NIH, USA and/or CorelDRAW X8 (Corel Corporation, Ottawa, Canada).

Figures were composed with CorelDRAW X8 (Corel Corporation, Ottawa, Canada) or with Microsoft Power Point (Microsoft Corporation).

Colocalization in ICC samples was measured by using ImageJ software and Pierson's Coefficient was calculated and detailed colocalization analysis were performed with the use of Coloc2 Plugin from Fiji (ImageJ software).

Statistical tests performed were students-two-tailed t-test, one-way-Analysis of Variance (ANOVA) and repeated-measures ANOVA for grouped analysis, followed by Dunnet's or Tukey's post-hoc tests for multiple comparison.

Data were expressed as mean \pm SEM and a $0.5 \%$ general significance level was defined, with significance levels as follows: ${ }^{*}$ : $<<0.05 ;{ }^{* *}: \mathrm{p}<0.01 ; * * * \mathrm{p}<0.001$. 
- IV -

RESULtS 


\subsection{UPTAKE OF RECOMBINANT ASYN SPECIES IN CULTURED CELLS}

\subsubsection{ASyn EnTERs H4 CELls AND Forms InTRACELLUlaR INCLUSIONS}

Several independent studies demonstrated that aSyn oligomers and pre-formed fibrils (PFFs) enter cultured cells and accumulate in the cytoplasm of cells $\mathrm{s}^{132,134}$. These findings have provided evidence on the spreading capacity of aSyn assemblies. However, it is still unclear where aSyn aggregation starts, how it proceeds in these models, and how relevant is the interaction of aSyn with membranes in the aggregation process.

In order to investigate the initial steps of the internalization process and aggregation of aSyn, we added monomers or fibrillar aggregates (fibrils) of aSyn to the cultured media of H4 human neuroglioma cells (Figure 16A).

First, we produced recombinant aSyn in bacteria and then generated monomeric or fibrillar species according to the methods described above. The species were characterized by $\mathrm{TEM}^{224}$ and by SDS-Page (Figure 16B). The preparation of aSyn monomers showed also the presence of a smaller amount of dimers, as illustrated by the band at $\sim 35 \mathrm{kDa}$. Due to contrasting studies reporting the ability of monomeric aSyn to passively enter cultured cells, we tested different concentration $(1 \mu \mathrm{M}$ and $5 \mu \mathrm{M})$ of these, while for aSyn fibrils we used the working concentration of $1 \mu \mathrm{M}$ (calculated based on the initial concentration of monomeric aSyn).

Then, recombinant proteins were added to the medium of $\mathrm{H} 4$ cells and the internalization of the exogenous aSyn into the cells was analysed by immunoblotting and by ICC after $24 \mathrm{~h}$ incubation.

Immunoblot analysis revealed that exogenous aSyn fibrils can be taken up by cultured cells. Surprisingly, the immunoblot clearly showed that monomeric aSyn is uptaken by $\mathrm{H} 4$ cells as well and that the internalization is dose-dependent (Figure 16C and 16D). Furthermore, ICC analysis demonstrated that, in cells exposed to monomeric aSyn, the protein accumulated in distinct perinuclear puncta, whereas in cells exposed to fibrils aSyn accumulated in larger cytosolic inclusions (Figure 16E). 
A

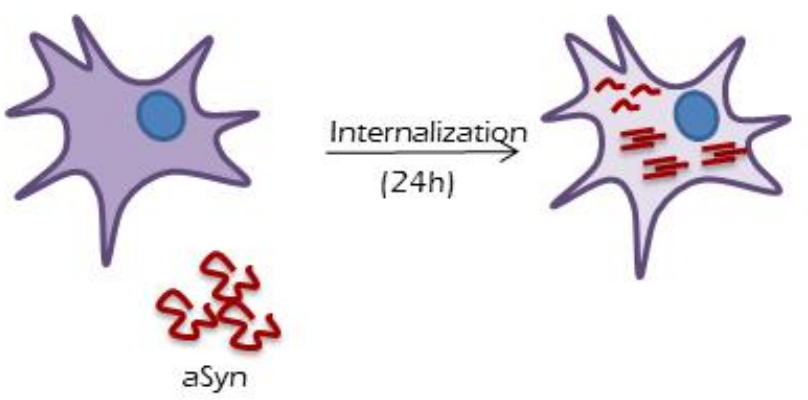

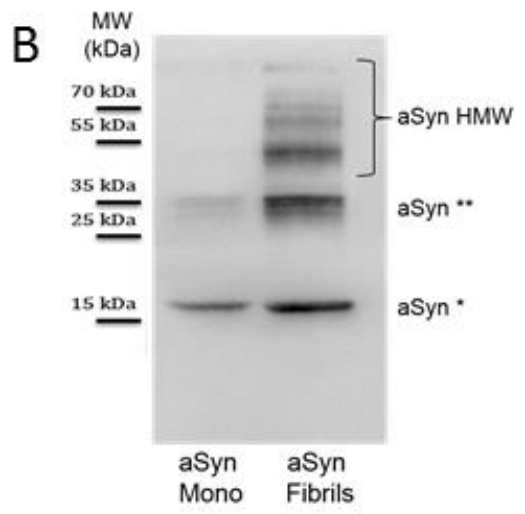

C

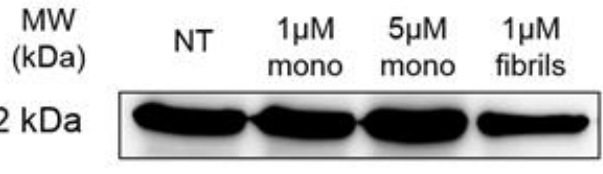

$15 \mathrm{kDa}$

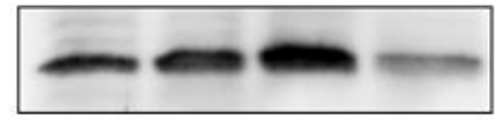

E
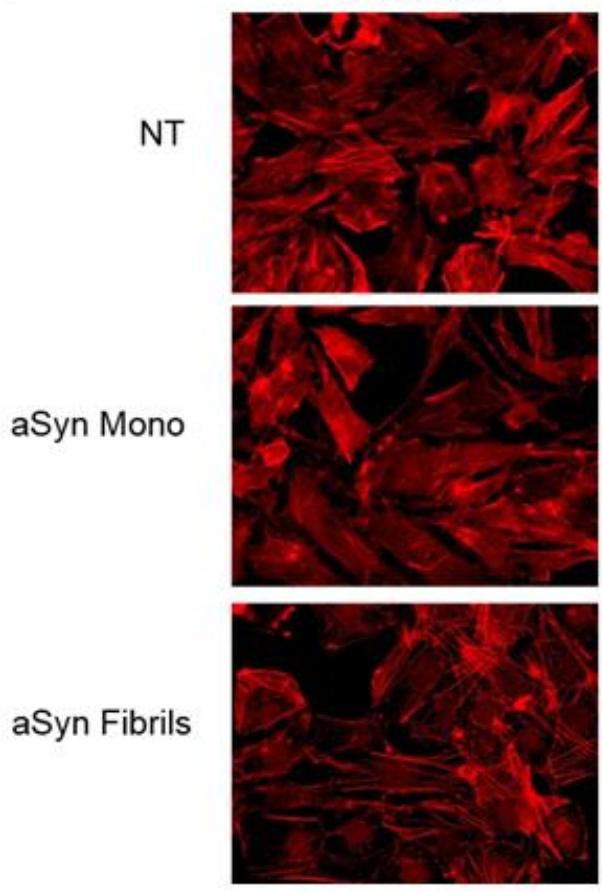

D

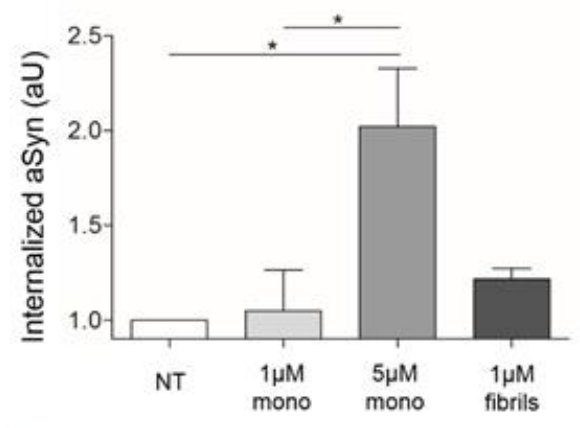

aSyn
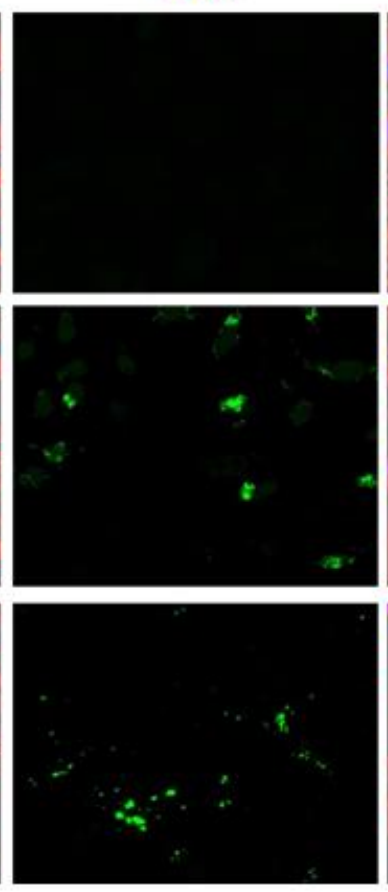

\section{Merge}
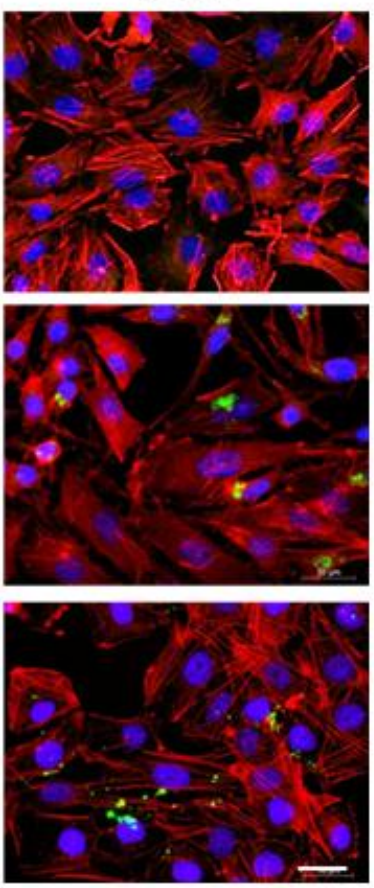

Figure 16. Recombinant aSyn monomers and fibrils are internalized by $\mathrm{H} 4$ cells.

(A) Recombinant aSyn monomers (aSyn Mono) or fibrils (aSyn Fibrils) were added to the cell culture medium and incubated for $24 \mathrm{~h}$. (B) SDS-PAGE and immunoblot analysis of the recombinant monomeric or fibrillar species of aSyn used in the experiments. The monomers show also the presence of a small fraction of dimers, as displayed by the faint band at $35 \mathrm{kDa}$. In the fibril preparation, one can observe the presence of higher molecular weight (aSyn HMW) species that are stable even on an SDS-PAGE. (C) WB of H4 cells after treatment with aSyn, confirming the internalization of aSyn monomers or fibrils as seen by the increase in the levels of aSyn in cells that were treated with monomers or fibrils. (D) Quantification of the immunoblot. Statistical test was performed using one-way ANOVA followed by Tukey's post-hoc tests. (E) ICC of cells treated using the conditions described. Scale bar: $30 \mu \mathrm{m}$. 


\subsubsection{ASyn Interacts With Plasma Membrane And Forms High Molecular Weight Species}

Whether aggregation of aSyn initiates from its lipid-bound $\alpha$-helical form or from its unstructured state remains controversial. In addition, the relationship between membrane binding and aSyn neurotoxicity is still unclear.

In order to understand whether the uptake of aSyn requires an interaction with the plasma membrane, we performed a cell surface biotinylation assay (Figure 17A and 17B). The biotinylation assay exploits the incubation of living cultured cells with nonpermeable biotin moieties to tag protein domains at the extracellular level or at the trans-membrane level. After extensive washing to eliminate the excess unbound biotin and cellular solubilisation in dedicated lysis buffer, biotinylated proteins were immobilized on a streptavidin-agarose resin and separated from the remaining, cytosolic proteins. After cellular lysis, resin capture and elution, two biochemical fractions were collected and analysed via WB: a biotinylated fraction, (containing membrane surface proteins and proteins interacting with plasma membrane) and a cytosolic fraction. Transferrin receptor and tubulin were used as positive controls for the biotinylated and the cytosolic fractions, respectively.

aSyn was found in the biotinylated fraction of $\mathrm{H} 4$ cells treated with monomers or fibrils, indicating that the interaction with plasma membrane is required for the uptake of exogenously added species in the form of both monomeric and fibrillar aSyn (Figure $17 \mathrm{~A}$ and 17B).

Once internalized in cultured cells, aSyn recombinant proteins are processed through some still indeterminate pathway that bring them to cluster in cytoplasmic located, punctuated structures, as displayed previously in Figure 16E.

To further investigate the nature of these inclusions, a dot blot analysis was performed. In this case, cell lysates of different conditions ( $\mathrm{H} 4$ cells untreated ( $\mathrm{H} 4$ cells NT) as a negative control, H4 cells incubated with aSyn monomers (H4 cells Mono) and H4 cells incubated with aSyn fibrils (H4 cells Fibrils)) were fractionated with the use of size-exclusion chromatography (SEC) technique. The collected fractions were then transferred in a nitrocellulose membrane and incubated with aSyn Ab.

Recombinant aSyn monomers were assessed by SEC in parallel, in order to establish the elution profile of this form of aSyn (Figure 18A and 18B).

As expected, the dot blot of $\mathrm{H} 4$ cells untreated did not show any positivity to $\mathrm{aSyn} \mathrm{Ab}$ (Figure 18C), whereas H4 cells treated with aSyn monomers show the presence of monomeric aSyn - fraction from $\mathrm{C} 1$ to $\mathrm{C} 4$, black box, Figure $18 \mathrm{C}$ - as reported in the 
chromatogram (Figure 18B). Interestingly, we could also detect aSyn in the latest fractions of the B lane - fractions from B6 to B15, red box, Figure 18C - indicating the presence of high molecular weight aSyn species as well.

Such species were also found in H4 cells treated with aSyn fibrils - fractions from B5 to B11, red box - while we could not detect any presence of monomeric species in this last one. (Figure 18C).

To further confirm the biochemical difference observed, we performed differential fractionation of the cell lysates using Triton X-100. Immunoblot analysis showed higher levels of Triton X-100-soluble aSyn in cells treated with monomers, and higher levels of Triton X-100-insoluble aSyn in cells treated with fibrils, consistent with the results of the SEC analysis (Figure 18D, left side). We also detected the formation of insoluble, high molecular weight aSyn species in cells treated with monomeric aSyn, suggesting that, upon internalization, aSyn monomers start to aggregated, to create high molecular species and to get insoluble (Figure 18D, right side). The presence of a smear on the sample treated with aSyn fibrils confirm the previous observation and reflect the insoluble character of the fibrils (Figure 18D).

Taken together, these results suggest that both monomeric and fibrillar aSyn can enter $\mathrm{H} 4$ cells and form high molecular weight species.

A

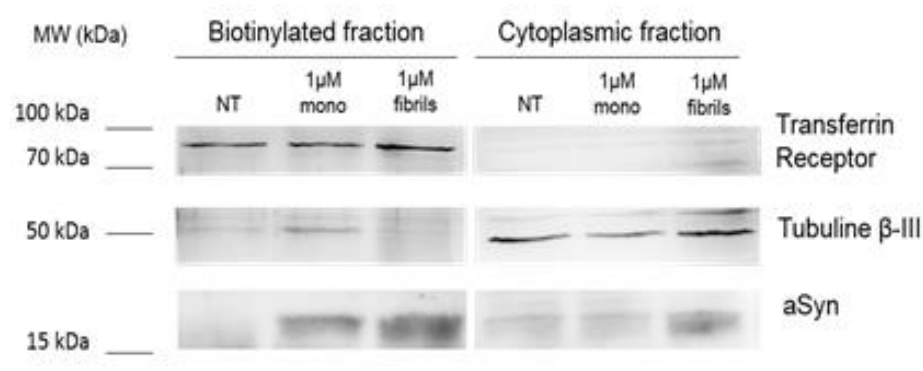

B

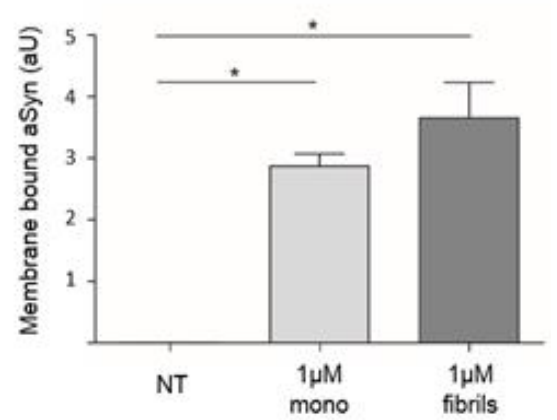

Figure 17. aSyn associates with membranes during the internalization process in $\mathrm{H} 4$ cells.

(A) Immunoblot of the biotinylation assay of $\mathrm{H} 4$ cells treated with aSyn monomers or fibrils. (B) Quantification of the levels of aSyn present in the biotinylated fraction (membrane-associated aSyn). Statistical test was performed using one-way ANOVA followed by Tukey's post-hoc test. 
A

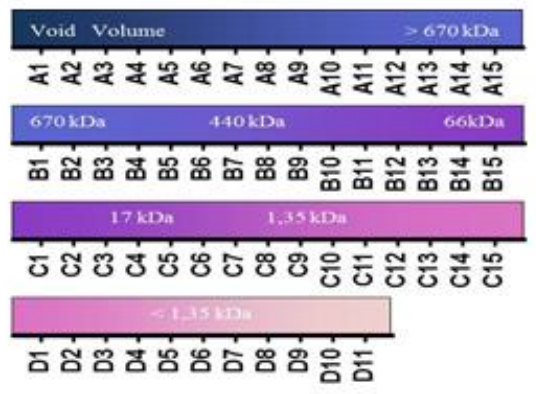

B

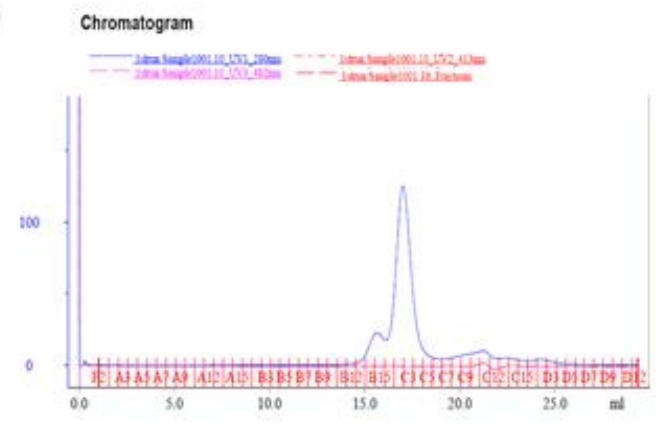

C

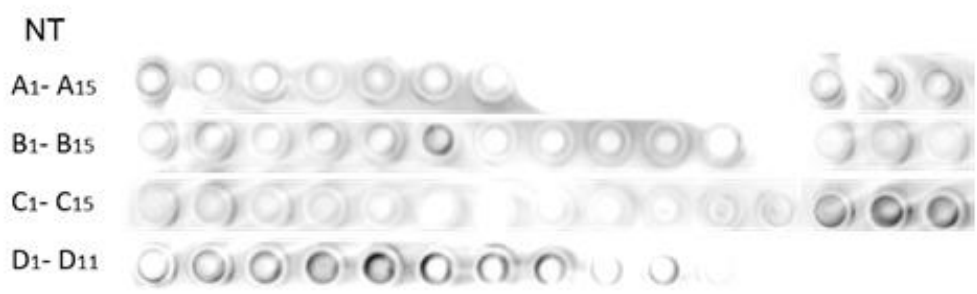

aSyn Mono

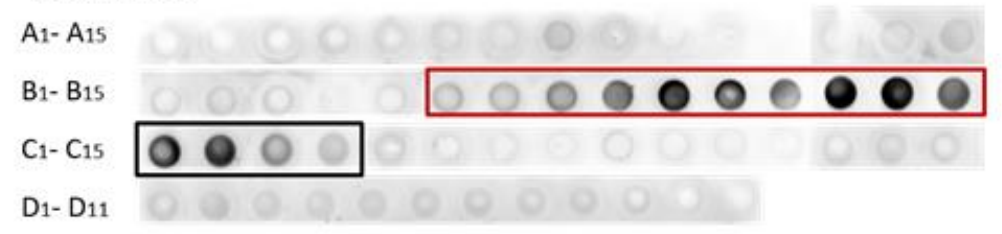

aSyn Fibrils

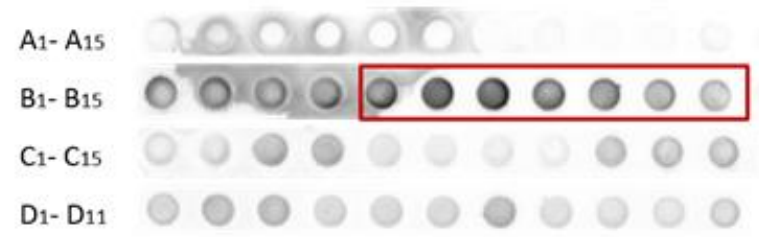

D

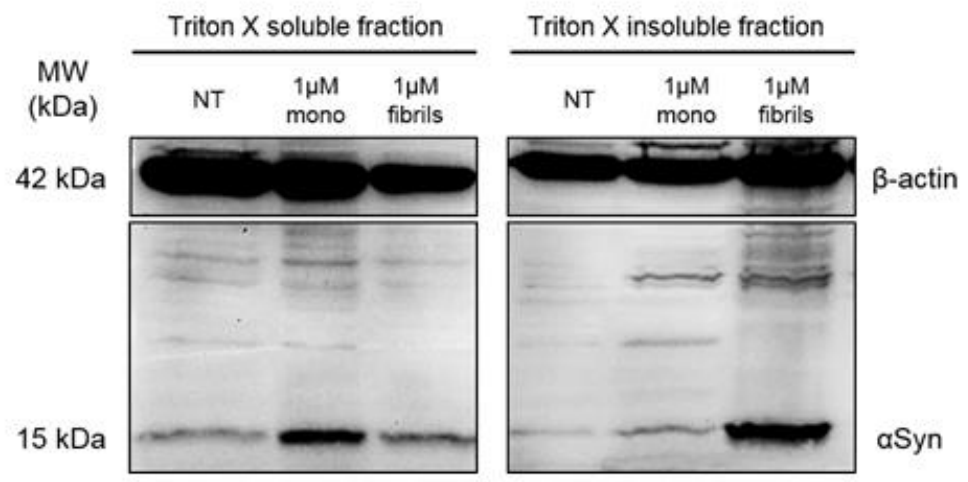

Figure 18. aSyn forms high molecular weight species in $\mathrm{H} 4$ cells.

(A) Protein separation scheme on Superose 6 10/300 column (B) Chromatogram of recombinant aSyn monomeric protein by using a Superose $610 / 300$ column

(C) Dot blot of the cell lysate of $\mathrm{H} 4$ cells untreated as a control, $\mathrm{H} 4$ cells treated with $1 \mu \mathrm{M}$ aSyn monomers and $\mathrm{H} 4$ cells treated with $1 \mu \mathrm{M}$ aSyn fibrils. The black rectangle highlights the presence of monomeric aSyn, located in the corresponding fraction after SEC, while the red boxes highlight the presence of high molecular weight species of aSyn. (D) Triton X-fractionation, with the soluble fraction (left panel) and the insoluble fraction (right panel) of $\mathrm{H} 4$ cells treated as described. 


\subsection{RAB-GTPASE PROTEINS MODUlate ASYN INTERNALIZATION AND AGGREGATION}

After confirming that exogenously added recombinant aSyn can be uptaken by $\mathrm{H} 4$ cells and interact with the plasma membrane, we investigated membrane trafficking machinery pathways that could potentially be involved in the process of internalization.

We then performed a screen of mammalian RAB-GTPase family proteins (RABs) to evaluate their effects on aSyn aggregation. The analysis was performed by overexpressing each one of the different RABs fused to GFP (Rab-GFP) (Table 2) in cultured cells. The day after transfection, $\mathrm{H} 4$ cells were treated with aSyn monomers or fibrils and, $24 \mathrm{~h}$ later, were processed for ICC.

From the initial analysis, a set of RABs that showed changes either in their subcellular localization or in the cellular interaction with aSyn recombinant protein was selected and further analysed (Table C).

As previously explained, the activity of RABs is regulated by guanine nucleotide exchange factors (GEFs), which catalyse the substitution of GDP for GTP and mediate their activation, and GTPase activating proteins (GAPs), which stimulate the intrinsic RABs activity, leading to their inactivation. Inactive, prenylated RABs bind to Guanine nucleotide dissociation inhibitors (GDIs) which keep them in a soluble state, in the cytosol, whereas the GDI displacement factors (GDFs) protein catalyse their dissociation from GDIs and their subsequent delivery to specific subcellular membranes ${ }^{225}$. Therefore, increase in the activity of RABs correspond to an increase in the membrane-bound, active state of the proteins, whereas an increased cytosolic, diffused localization pattern will reflect the inactive state of the protein.

After a screening performed on the mammalian RABs, we displayed how the presence of aSyn monomers or fibrils can influence the distribution of selected RABs (Table C). 


\begin{tabular}{|c|c|c|c|c|c|c|}
\hline \multirow[t]{2}{*}{ RABs } & \multirow[t]{2}{*}{ Localization } & \multirow[t]{2}{*}{ Function } & \multicolumn{2}{|c|}{$\begin{array}{c}\text { H4 cells + aSyn } \\
\text { monomers }\end{array}$} & \multicolumn{2}{|c|}{$\begin{array}{c}\text { H4 cells + aSyn } \\
\text { fibrils }\end{array}$} \\
\hline & & & Colocalization & Morphology & Colocalization & Morphology \\
\hline Rab 1A, 1B & ER, Golgi & $\begin{array}{l}\text { ER to Golgi, } \\
\text { Intra-Golgi }\end{array}$ & No & $\begin{array}{c}11 \% \text { more } \\
\text { RAB-vesicles }\end{array}$ & No & $\begin{array}{c}9 \% \text { more } \\
\text { RAB-vesicles }\end{array}$ \\
\hline $\mathrm{Rab} 3 \mathrm{~A}$ & $\begin{array}{l}\text { Secretory vesicles, } \\
\text { plasma membrane }\end{array}$ & $\begin{array}{l}\text { Exocytosis, } \\
\text { neurotrasmitter } \\
\text { release }\end{array}$ & + & $\begin{array}{c}7 \% \text { more } \\
\text { RAB-vesicles }\end{array}$ & + & $\begin{array}{c}15 \% \text { more } \\
\text { RAB-vesicles }\end{array}$ \\
\hline $\mathrm{Rab} 4 \mathrm{~A}$ & Early endosome & $\begin{array}{l}\text { Protein recycling/ } \\
\text { transport to plasma } \\
\text { membrane }\end{array}$ & +++ & $\begin{array}{c}51 \% \text { more } \\
\text { RAB-vesicles }\end{array}$ & No & $\begin{array}{c}42 \% \text { more } \\
\text { RAB-vesicles }\end{array}$ \\
\hline Rab 5A & $\begin{array}{l}\text { PM, CCVs, early } \\
\text { endosome }\end{array}$ & $\begin{array}{l}\text { Early endosome } \\
\text { fusion }\end{array}$ & +++ & $\begin{array}{c}32 \% \text { more } \\
\text { RAB-vesicles }\end{array}$ & No & $\begin{array}{c}35 \% \text { more } \\
\text { RAB-vesicles }\end{array}$ \\
\hline Rab 6A & Golgi & $\begin{array}{l}\text { Endosome to Golgi, } \\
\text { intra-Golgi transport } \\
\text { Golgi to ER }\end{array}$ & No & $\begin{array}{c}46 \% \text { more } \\
\text { RAB-vesicles }\end{array}$ & No & $\begin{array}{c}41 \% \text { more } \\
\text { RAB-vesicles }\end{array}$ \\
\hline Rab 7 & $\begin{array}{l}\text { Late endosome, } \\
\text { lysosome, } \\
\text { melanosomes, } \\
\text { phagosome }\end{array}$ & $\begin{array}{l}\text { Late endosome to } \\
\text { lysosome }\end{array}$ & +++ & $\begin{array}{c}54 \% \text { more } \\
\text { RAB-vesicles }\end{array}$ & No & $\begin{array}{c}50 \% \text { more } \\
\text { RAB-vesicles }\end{array}$ \\
\hline Rab 9A & Late endosome & Endosome to TGN & +++ & $\begin{array}{l}\text { No changes in } \\
\text { the distribution } \\
\text { pattern }\end{array}$ & No & $\begin{array}{c}\text { No changes in } \\
\text { the distribution } \\
\text { pattern }\end{array}$ \\
\hline Rab 15 & $\begin{array}{l}\text { Early/sorting } \\
\text { endosome, recycling } \\
\text { endosome }\end{array}$ & $\begin{array}{l}\text { Sorting endosome/ } \\
\text { recycling endosome } \\
\text { to plasma membrane }\end{array}$ & No & $\begin{array}{l}40 \% \text { smaller } \\
\text { RAB-vesicles }\end{array}$ & + & $\begin{array}{l}23 \% \text { smaller } \\
\text { RAB-vesicles }\end{array}$ \\
\hline Rab 25 & Recycling endosome & $\begin{array}{l}\text { Recycling endosome } \\
\text { to plasma membrane }\end{array}$ & No & $\begin{array}{c}40 \% \text { more } \\
\text { RAB-vesicles, } \\
15 \% \text { more } \\
\text { cytosolic RAB }\end{array}$ & No & $\begin{array}{c}18 \% \text { more } \\
\text { RAB-vesicles, } \\
48 \% \text { more } \\
\text { cytosolic RAB }\end{array}$ \\
\hline Rab 27B & Melanosome & Exocytosis & + & $\begin{array}{c}41 \% \text { more } \\
\text { RAB-vesicles }\end{array}$ & No & $\begin{array}{c}25 \% \text { more } \\
\text { RAB-vesicles }\end{array}$ \\
\hline $\begin{array}{l}\text { Rab 33A, } \\
33 \mathrm{~B}\end{array}$ & $\begin{array}{l}\text { Golgi, dense-core } \\
\text { vesiscles }\end{array}$ & $\begin{array}{l}\text { Autophagosome } \\
\text { formation }\end{array}$ & No & $\begin{array}{c}18 \% \text { more } \\
\text { RAB-vesicles }\end{array}$ & No & $\begin{array}{c}35 \% \text { more } \\
\text { RAB-vesicles }\end{array}$ \\
\hline
\end{tabular}

\section{TABLE C. Selected RABs from the screen.}

RAB-GTPase family members selected in a screen where we assessed alterations in the subcellular distribution of the RAB protein or the colocalization with aSyn in cells treated with aSyn monomers or fibrils. In the column "morphology", an "11\% more RAB-vesicles" statement means that in the $11 \%$ of the cells analysed, the localization of RABs is more vesicular (suggesting an increase of $11 \%$ in the active, GTP-bound RAB protein) compared to the localization pattern shown in naive cells. In contrast, the statement " $15 \%$ more cytosolic RAB" indicates that, in this case, $15 \%$ of the cells analysed showed an increase in the cytosolic, diffuse localization of $\mathrm{RAB}$ protein when compared to the naive cells (suggesting an increase of $15 \%$ in the inactive, GDP-bound RAB protein). 


\subsubsection{ASyn Partially Colocalizes With Rab 5A And Rab 7}

The data from our screen revealed that, among the selected RABs, the ones that show the strongest colocalization with aSyn or changes in the morphology are Rab 4A, Rab 5A and Rab 7, especially if we consider their interaction with monomeric aSyn more than with the fibrillar species (Table C).

This findings match very well with the hypothesis that aSyn is internalized via an active process and that get processed inside the cells in vesicular compartments such as endosomes and lysosomes, especially if we consider the localization and function of the above-mentioned RABs members ${ }^{162}$ (Table A).

$\mathrm{Rab} 4 \mathrm{~A}$ and $\mathrm{Rab} 5 \mathrm{~A}$ are localized in the early endosome, contributing to the protein recycling/transport to the plasma membrane and early endosome fusion, respectively, while Rab 7 is localized in the late endosome, lysosome and phagosome, contributing to the late endosome to lysosome fusion process.

We then analysed the results obtained from the first screening in greater detail.

First, we assessed the degree of colocalization of aSyn and Rab5A-GFP or Rab7GFP in cells treated with aSyn monomers or fibrils (Figure 19A). The colocalization was quantified using the Coloc2 plugin of ImageJ Software (Figure 19B).

We observed a strong colocalization between the aSyn-positive inclusions formed after the treatment with aSyn monomers (in red) and Rab 5A-GFP vesicles (in green) (Figure 19A, left column, central panel), as well as a partial - although weaker - colocalization of the aSyn newly formed aSyn inclusions with Rab 7-GFP (Figure 19A, right column, central panel ). On the other side, the colocalization was not observed when cells were treated with aSyn fibrils. This supports the idea that the internalization and sorting of aSyn monomers and fibrils is different, as one might expect given their distinct biochemical properties.

The Pearson's coefficients obtained from the analysis of several samples show that aSyn monomers, but not aSyn fibrils, once internalized, give rise to inclusions that partially colocalize with Rab 5A-GFP and with Rab 7-GFP, indicating that the endocytic pathways, as well as the lysosomal one are involved in the internalization of aSyn monomers (Figure 19B). 
A
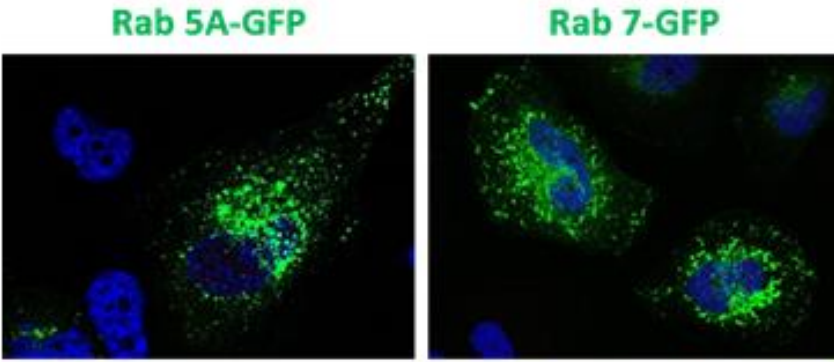

NT
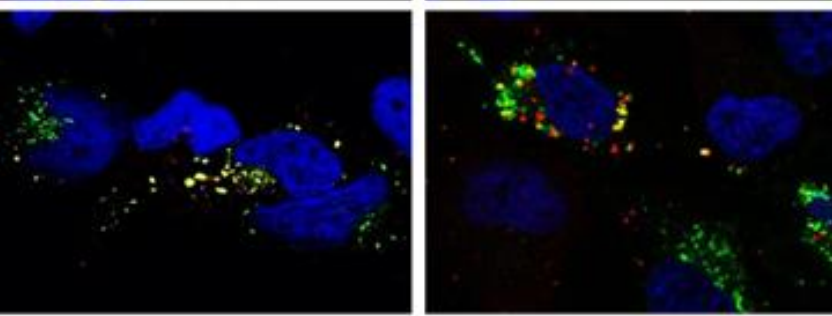

aSyn Mono
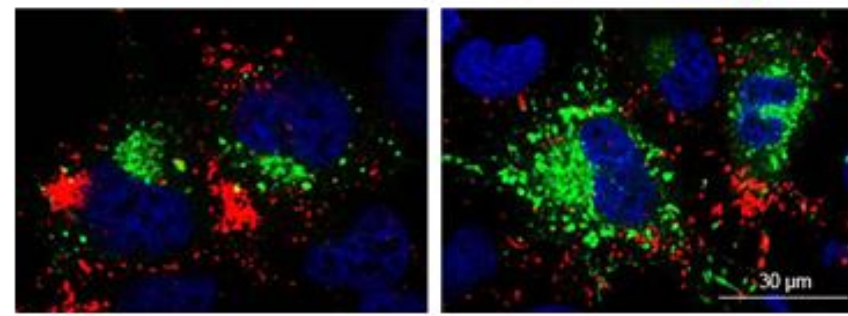

aSyn Fibrils

B
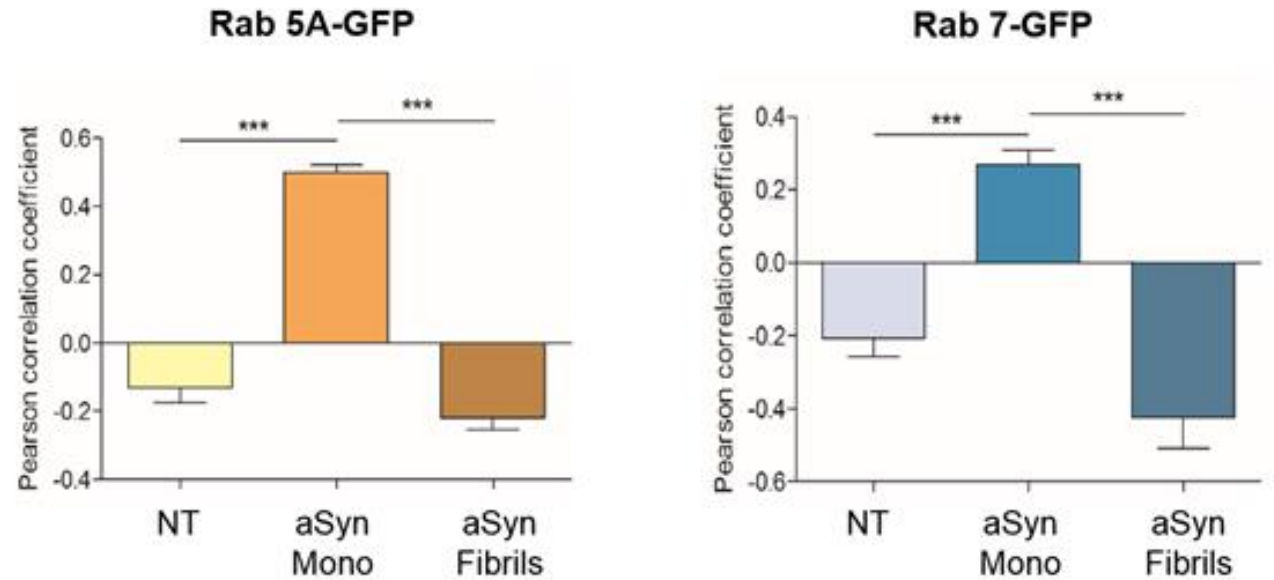

Figure 19. aSyn partially colocalizes with Rab 5A-GFP and Rab 7-GFP in H4 cells .

(A) ICC on H4 cells transfected with Rab 5A-GFP (right side of the panel) or with Rab 7-GFP and treated with $1 \mu \mathrm{M}$ aSyn monomers or $1 \mu \mathrm{M}$ aSyn fibrils. (B) Pearson correlation coefficient confirming colocalization of aSyn with Rab $5 \mathrm{~A}$ or Rab 7 in cells treated with aSyn monomers, but not with fibrils. Scale bar: $30 \mu \mathrm{m}$ 


\subsubsection{ASYN Forms INCLUSIONS SURROUNDED By RAB 4A Positive VesicLes}

Next, we examined more in details the colocalization and the morphological changes of Rab 4A-GFP in the presence of aSyn recombinant proteins.

On the early endosomal membrane Rab 4 and Rab 5 occupy distinct domains, where Rab 5 marks the cisternal domain and Rab 4 concentrates in patches that undergo fission from the cisternae, called tubular domains. These tubular subdomains are highly dynamics, giving rise to multiple classes of carrier vesicles that are targeted to different destinations ${ }^{226}$.

Based on the ICC results obtained, when H4 cells are transfected with Rab 4AGFP and treated with aSyn fibrils, no effects on the morphology of the small GTPase protein, neither colocalization with aSyn can be appreciated (Figure 20). On the other side, when $\mathrm{H} 4$ cells are transfected with Rab 4A-GFP and treated with aSyn monomers, it is possible to observe a dramatic increase in the endosome size, as well as a massive internalization of aSyn monomers, that accumulate in inclusions surrounded by those big, abnormal Rab 4A positive vesicles (Figure 20, central panel on the top and lower panels).

We found that the internalization of aSyn monomers is increased by the overexpression of $\mathrm{Rab} 4 \mathrm{~A}$, and that when aSyn monomers are added to cells, aSyn forms inclusions surrounded by Rab $4 \mathrm{~A}$. We also observed a drastic change in the morphology of Rab 4A positive vesicles, and therefore of the early endosome size, suggesting that the exposure to aSyn monomers altered the normal biology of Rab4A and, therefore, the early endocytosis- related process and vesicle formation. 


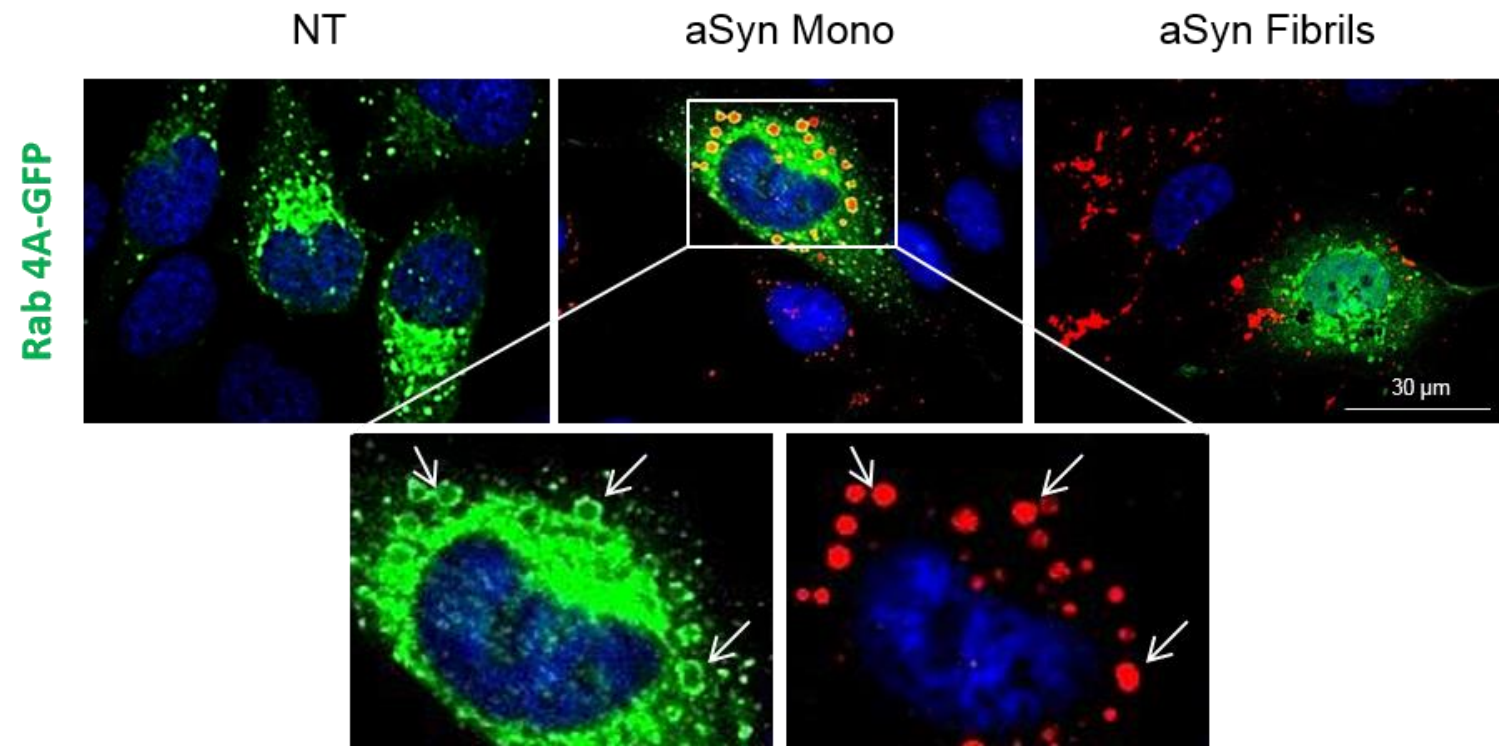

Figure 20. aSyn forms inclusions surrounded by $\mathrm{Rab} 4 \mathrm{~A}$ positive vesicles in $\mathrm{H} 4$ cells

ICC on cells transfected with Rab 4A-GFP and treated with $1 \mu \mathrm{M}$ of aSyn monomers or fibrils. Inset: zoom and separated channels of Rab 4A-GFP aSyn. Arrows point to the large inclusions aSyn (in red, panel on the right) matching with the GFP-positive Rab 4A vesicles (in green, panel on the left). Scale bar: $30 \mu \mathrm{m}$

\subsection{ASYN MEMBRANE BINDING PROPERTIES}

Considering the results obtained, we decided to focus on treatments with aSyn monomers in order to further study the interaction with membrane at a molecular and structural level.

\subsubsection{Membrane Binding Properties Are Essential For The INTERNALIZATION AND THE INCLUSIONS FORMATION OF ASYN IN H4 CELLS}

Recent findings support the hypothesis that aSyn multimerizes on lipid membranes ${ }^{53}$, and this raised the question whether such $\alpha$-helical assemblies directly convert into $\beta$ strand containing neurotoxic properties or whether it is the unstructured, soluble aSyn monomeric species that actively trigger the formation to aggregates inside cells, during the processing and the compartmentalization in different organelles and the interaction with effector proteins. 
Our findings support the second hypothesis, suggesting that monomeric aSyn, once internalized, start to accumulate in endosomal compartments and to form high molecular weight species.

In order to address this central question, we analyzed the rate of internalization of different aSyn mutants that have altered membrane binding properties. In particular, we used the aSyn A30P familial mutant, known to display weaker binding to membranes, and an artificial mutant (A11P/V70P) designed to severely impair membrane binding 227,228 .

To assess the ability of the different aSyn variants to bind to the plasma membrane, we performed a biotinylation assay (Figure 21A and 21B). We detected a clear trend in the amount of protein present in the biotinylated fractions that reflected the different membrane binding properties of the aSyn mutants (aSyn A30P and aSyn A11P/V70P). We also detected a consistent trend in the levels of aSyn dimeric species in the biotinylated fraction, suggesting that membrane binding is also important for dimerization and aggregation of aSyn.

Next, we tested the ability of the three different mutants to enter cells and to form inclusions, via ICC (Figure 21C) and immunoblotting analysis (Figure 21D and 21E). The ICC revealed a significant reduction in the accumulation of aSyn in inclusions in cells treated with A30P or A11P/V70P aSyn mutants when compared with cells treated with WT (Figure 21C), consistent with a difference in the internalization of the mutants.

The immunoblot analysis allowed us to further quantify the internalization of this different monomeric aSyn species, showing that despite they preserve the ability to partially dimerize when purified in vitro, , their ability to get uptaken in cultured cells is drastically reduced (Figure $21 \mathrm{D}$, low panel).

Taken together, these results suggest that membrane binding properties are essential for the internalization, and therefore, for the formation of intracellular aSyn inclusions in cultured cells. 
A

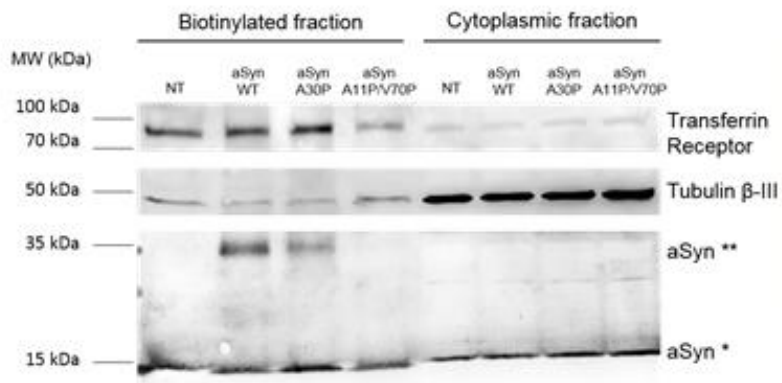

C
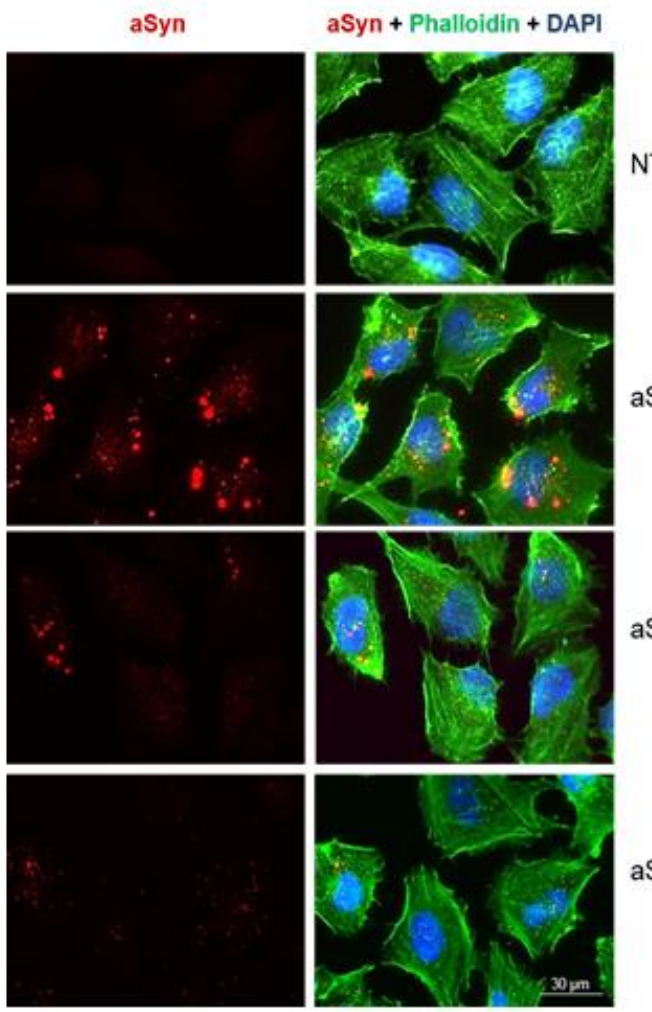

aSyn A30P
B

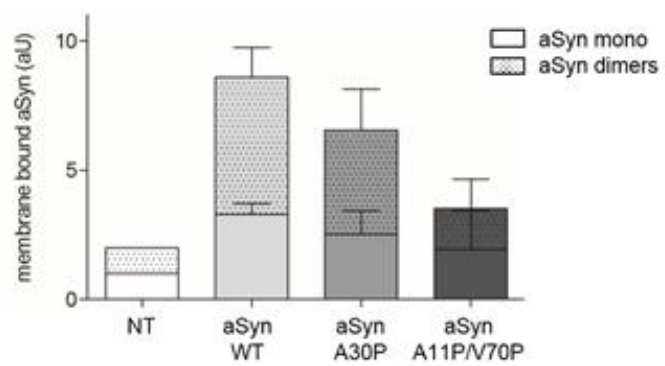

D
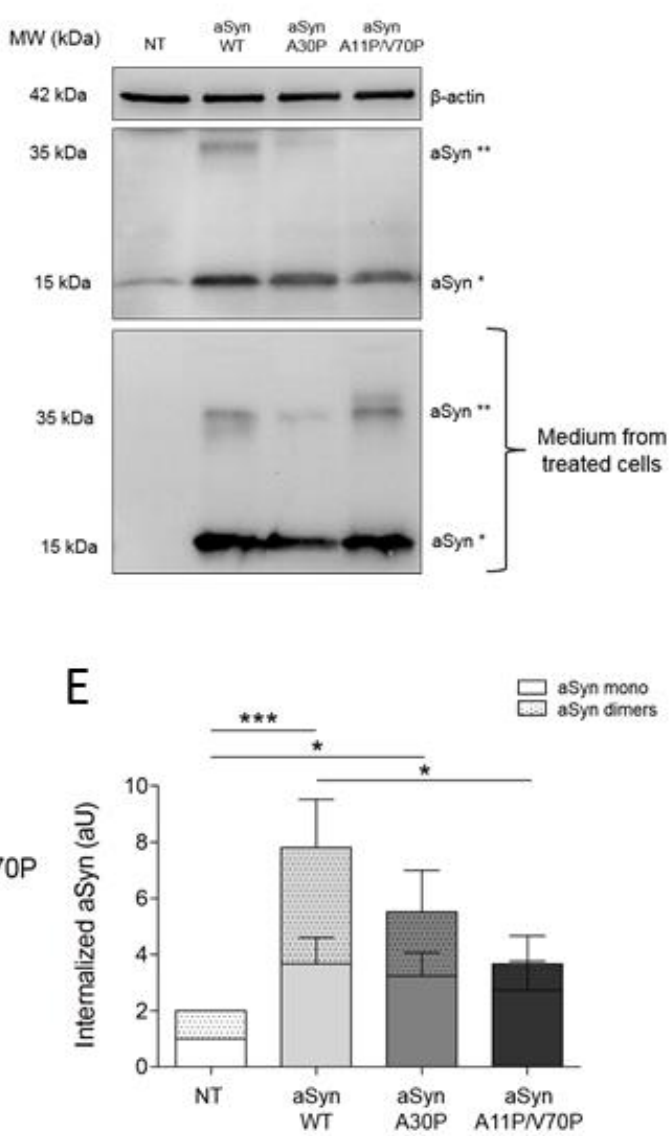

Figure 21. Membrane binding is essential for the internalization and inclusion formation of aSyn in $\mathrm{H} 4$ cells.

(A and B) Immunoblot and quantification of the comparison of WT aSyn and two mutants (A30P and $\mathrm{A} 11 \mathrm{P} / \mathrm{V} 70 \mathrm{P}$ ) with different membrane-binding properties using a biotinylation assay. Dotted bars refer to the band corresponding to aSyn dimers (aSyn**), and clear bars refer to aSyn monomers (aSyn*). (C) ICC and (D) immunoblotting of non-treated (NT) cells, or cells treated with WT, A30P, or A11P/V70P aSyn for 24h. Scale bar: $30 \mu \mathrm{m}$. (E) Quantification of the immunoblot in panel D. Dotted bars refer to the band corresponding to aSyn dimers (aSyn**), and clear bars refer to aSyn monomers (aSyn*). Statistical tests were performed using one-way ANOVA with repeated-measures for grouped analysis, followed by Tukey's post-hoc tests. Data were expressed as mean \pm SEM and a $0.5 \%$ general significance level was defined, with significance levels as follows: $*: \mathrm{p}<0.05 ; * *: \mathrm{p}<0.01 ; * * *: \mathrm{p}<0.001$. Statistical significance is indicated with the symbol "\#" for the monomers, "+" for the dimers, and "*” for the summa between monomers and dimers. 


\subsubsection{ASyn A11P/V70P Is UnABLE To BIND MEMBRANES}

Since the discovery of the A30P familial mutation, various groups have extensively studied the effect of the mutation on the biochemical and biophysical properties of the protein.

The A11P/V70P artificial mutant was designed as a lipid-binding deficient mutant, but has only been studied in vitro, not in cell models.

First, we confirmed the previous findings and performed an in vitro membrane binding assay using small unilamellar vesicles (SUVs), and then we tested the ability of the mutant to get internalized and form inclusion by adding different amounts of A11P/V70P to H4 cells (Figure 22).

We generated SUVs from a 5:3:12 mixture of DOPE:DOPS:DOPC.

NMR spectroscopy revealed that aSyn interacted with DOPE:DOPS:DOPC liposomes as previously described ${ }^{229,230}$ (Figure 22A, panel on the left). In contrast to aSyn WT, the aSyn A11P/V70P mutant induced a drastic reduction (more than 80\%) of the signal broadening starting from the residue 11 (where the first Pro mutation is located) until residue 140 (Figure 22A, panel on the right), confirming the impairment in membrane binding.

Next, we tested the effect of aSyn A11P/ V70P in our cellular model. To do this, we tested different concentration of aSyn A11P/V70P $(1 \mu \mathrm{M}$ and $5 \mu \mathrm{M})$ and we compared this with aSyn WT $(1 \mu \mathrm{M}$ and $5 \mu \mathrm{M})$. We also used H4 cells transfected with Rab 4AGFP, as we observed an increased internalization of aSyn in these cells.

As it is clearly shown from the immunoblot analysis (Figure 22B and 22C), as well as from the ICC (Figure 22D), we observed that the internalization of aSyn WT and the formation of inclusions are dose-dependent, and that these are further increased upon overexpression of Rab 4A.

On the other side, the internalization of aSyn A11P/V70P was negligible when compared to aSyn WT, and did not increase with higher concentrations nor with the overexpression of Rab 4A-GFP.

The results obtained confirm that aSyn A11P/V70P is a valid model to test the effect of membrane binding on the internalization and inclusion formation of aSyn proteins in cultured cells. 
A

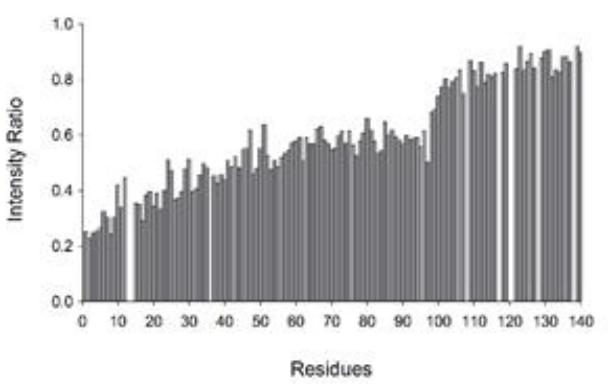

Membrane Binding properties
aSyn A11P V70P / SuVs [ratio 1:100

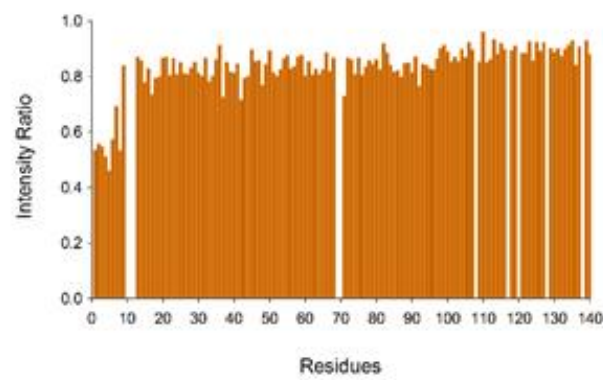

B

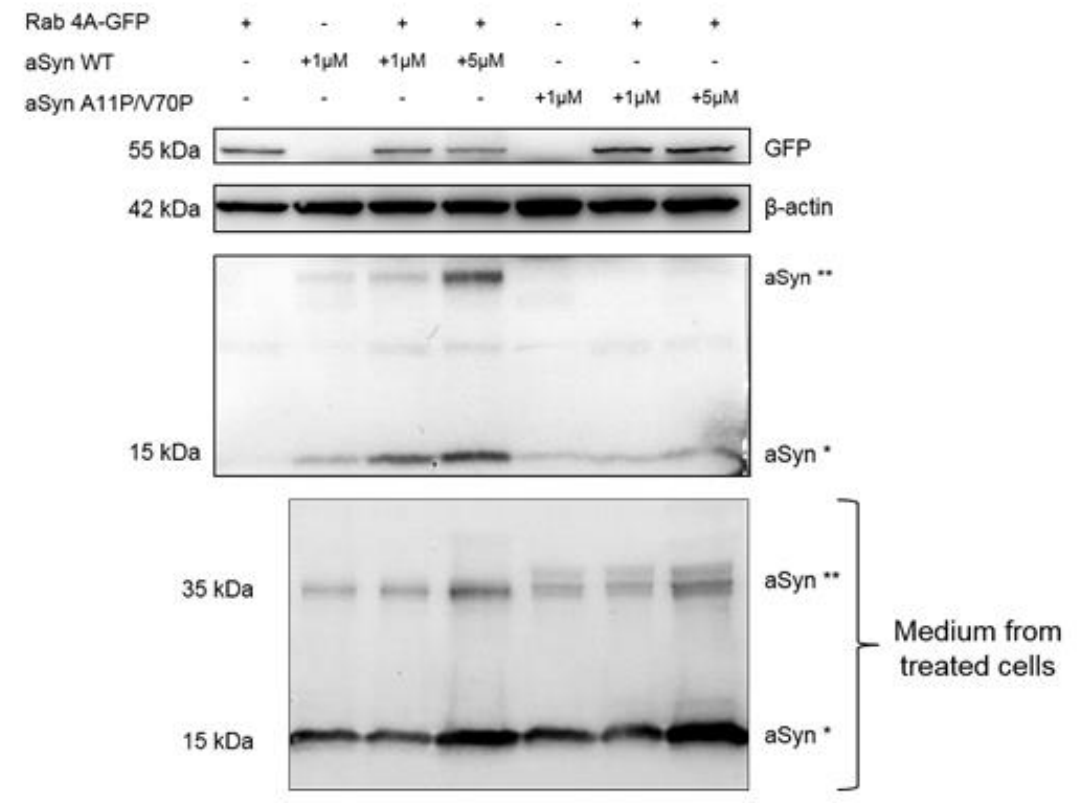

C

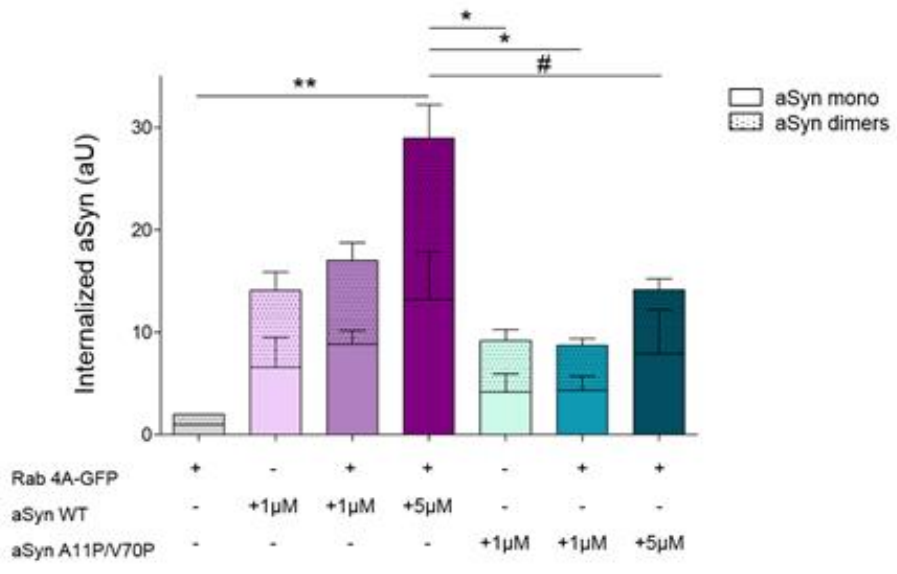


D

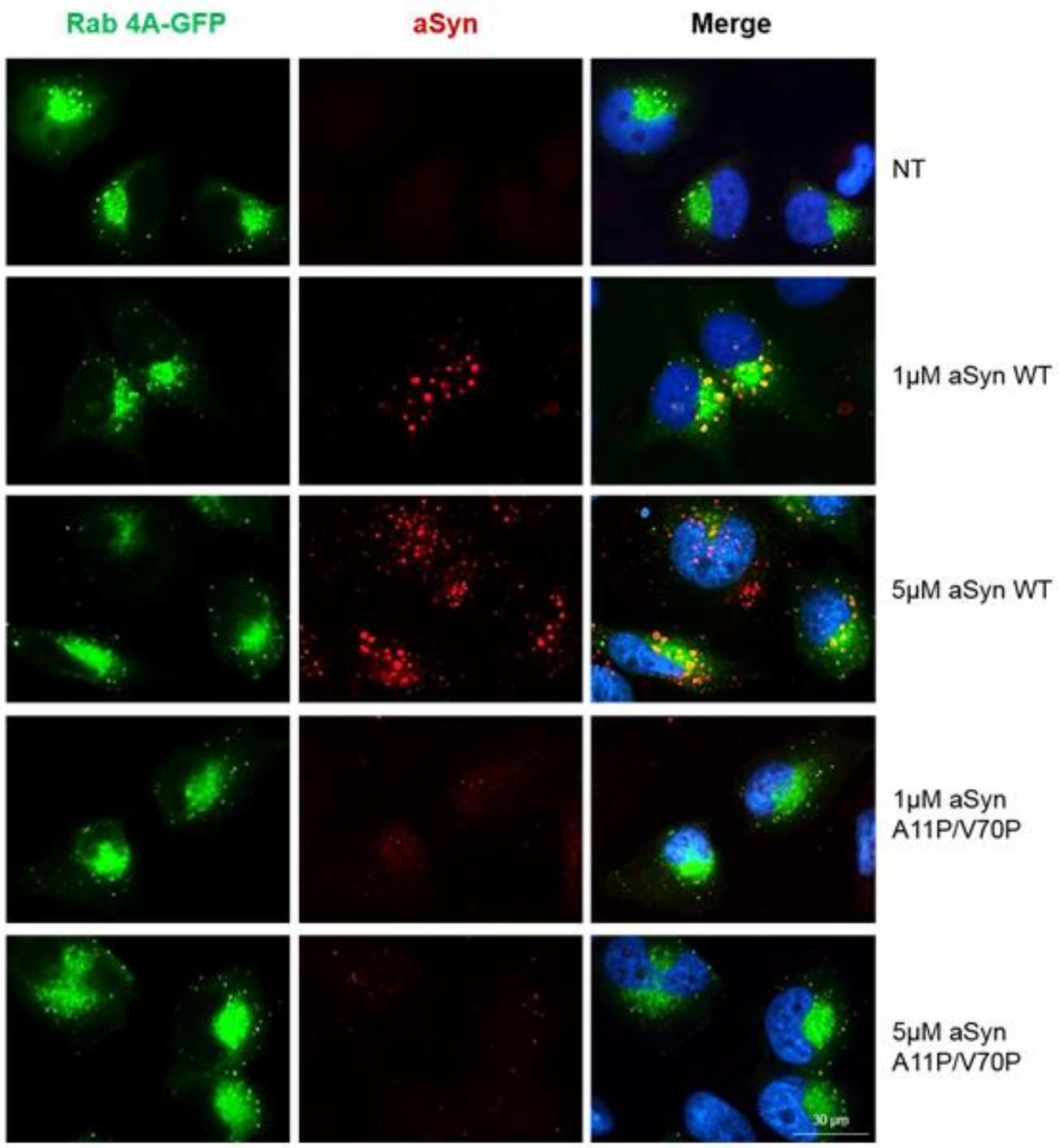

Figure 22. Characterization of aSyn A11P/V70P mutant.

(A) Membrane binding properties of WT (left) and of A11P/V70P aSyn (right) in the presence of artificial small unilamellar vesicles membranes (SUVs) [1:100 protein:SUVs ratio]. (B) Immunoblotting of Rab 4A-GFPexpressing cells treated with $1 \mu \mathrm{M}$ or $5 \mu \mathrm{M}$ of WT or A11P/V70P aSyn. (C) Quantification of the immunoblots. Dotted bars refer to the band corresponding to aSyn dimers ( $\left.\mathrm{aSyn}^{* *}\right)$, and clear bars refer to aSyn monomers $\left(a^{2} n^{*}\right)$. Statistical tests were performed using one-way-analysis of variance (ANOVA) with repeated-measures for grouped analysis, followed by Tukey's post-hoc tests. Data were expressed as mean \pm SEM and a $0.5 \%$ general significance level was defined, with significance levels as follows: * $\mathrm{p}<0.05$; $* *$ : $\mathrm{p}<0.01$; $* * *$ : $\mathrm{p}<$ 0.001 . The significance is showed with the symbol "\#" for the monomers, with the symbol "+" for the dimers and with the symbol "*” for the summa between monomers and dimers. (D) ICC of $\mathrm{H} 4$ cells transfected with Rab 4A-GFP and treated with $1 \mu \mathrm{M}$ or $5 \mu \mathrm{M}$ of aSyn WTand aSyn A11P/V70P. Scale bar: $30 \mu \mathrm{m}$. 


\subsubsection{ASyN A30P AND ASYN A11P/V70P ARE LESS INTERNALIZED IN RAB 4A AND RAB 5A TRANSFECTED CELLS}

In order to further investigate whether the membrane binding properties of aSyn could affect not only in the internalization but also the processing and metabolism of the protein via the endocytic pathway, we tested the ability of the three different mutants to get internalized in cells overexpressing either Rab 4A-GFP or Rab 5A-GFP. We also tested our aSyn recombinant monomers in $\mathrm{H} 4$ cells transfected with a constitutively active (CA) form of Rab 5A-GFP (Rab 5ACA-GFP) so to explore whether a further deregulation of the endocytic pathway could somehow help or overcome the lack of binding properties of these aSyn mutant forms (Figure 23).

The ICC of H4 cells transfected with Rab 4A-GFP show a massive internalization of aSyn WT, giving rise to these characteristic big inclusions surrounded by Rab 4A positive vesicles as already described, and a significant, progressive decrease of the rate of internalization of aSyn A30P and aSyn A11P/V70P (Figure 23A and 23B). Curiously, the amount of monomeric forms versus dimeric forms internalized in this case is higher than the one showed from the not-transfected H4 cells (Figure 21D and 21E) as well as from the one showed by $\mathrm{H} 4$ cells transfected with Rab 5A-GFP, to possibly indicate the effect that the overstimulation of the tubular subdomain can have in the vesicle formation and in the protein internalization at plasma membrane level. The same trend, although with less significance, is observed in the ICC of H4 cells transfected with Rab 5A-GFP indicating, once again, that the overexpression of Rab $5 \mathrm{~A}$ (as well as of Rab 4A) and the consequential overstimulation of the early steps of the endosome formation can surely improve the amount of aSyn internalized, but only if the membrane binding properties of the protein are preserved (Figure 23C and 23D). In cells overexpressing Rab 5A, we observed an increase in the levels of aSyn dimeric species, to indicate the possible role played by endosomes in the dimerization process and aSyn aggregation formation.

Finally, we analysed the rate of internalization of aSyn mutants in $\mathrm{H} 4$ cells transfected with Rab 5ACA-GFP (Figure 23E and 23F). As in this case the GTPase protein Rab $5 \mathrm{~A}$ is deregulated, resulting in a permanent activation, the amount of aSyn internalized results to be higher compared to the one observed in $\mathrm{H} 4$ cells transfected with Rab 5A, further confirming the role of the endocytic pathway in the internalization and processing of aSyn. 
A

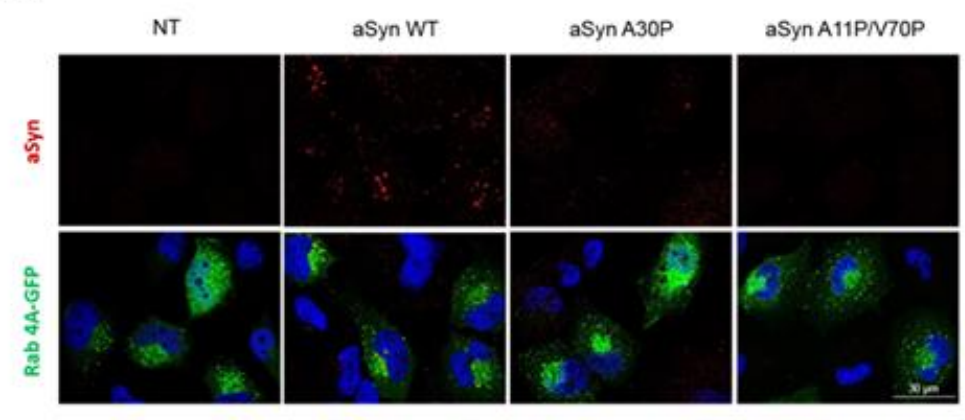

C

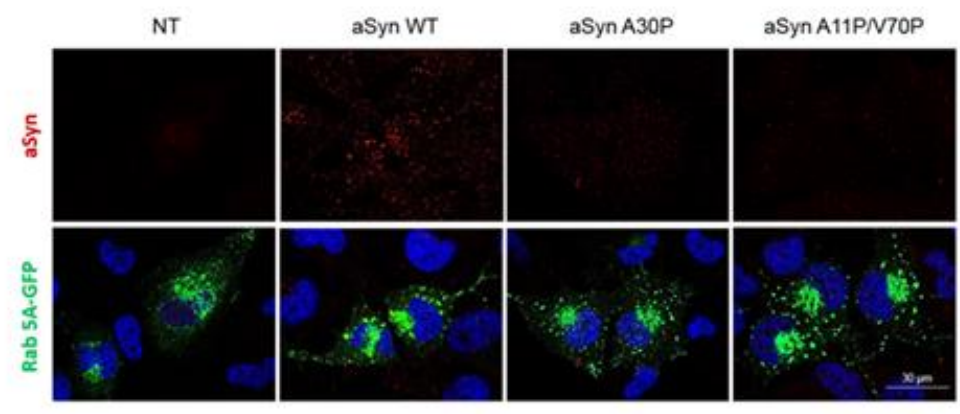

$E$

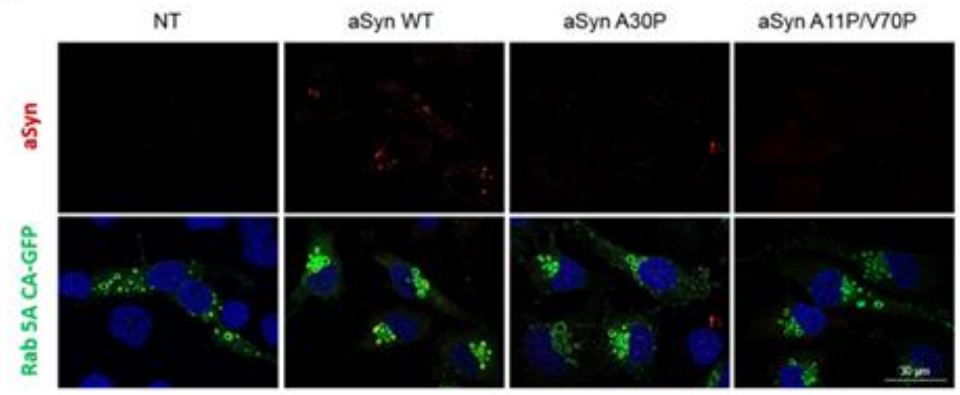

B

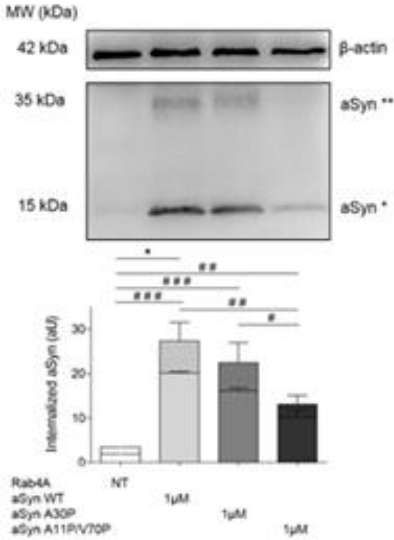

D
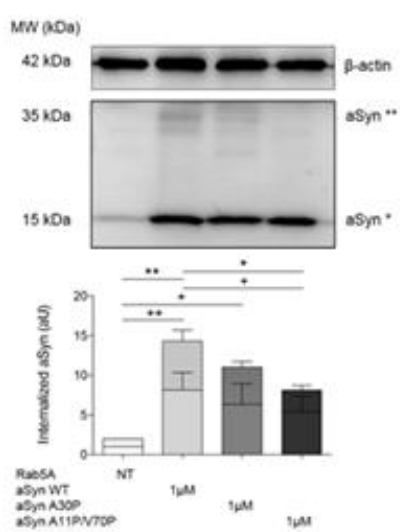

$\mathrm{F}$

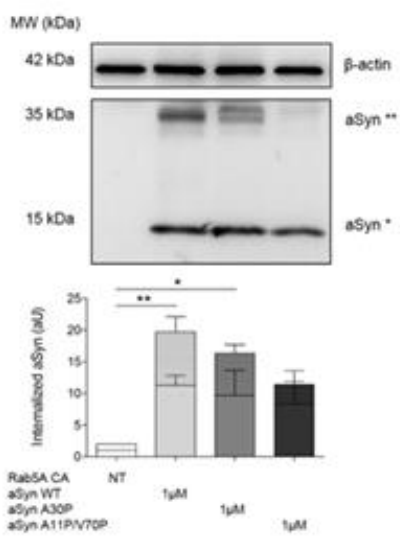

Figure 23. aSyn A30P and A11P/V70P are less internalized in Rab 4A-GFP, Rab 5A-GFP and Rab 5ACAGFP transfected cells compared to aSyn WT.

(A) ICC and (B) Immunoblotting of cells transfected with Rab 4A-GFP and treated as in Figure 5. (C and D) ICC and Immunoblotting of cells transfected with Rab 5A-GFP and treated as above. (E and F) ICC and Immunoblotting of cells transfected with Rab 5ACA-GFP (constitutively active) and treated as above. (B, D and F, lower panels) Quantifications of the immunoblots. Dotted bars refer to the band corresponding to aSyn dimers $\left(\mathrm{aSyn}{ }^{* *}\right)$, and clear bars refer to aSyn monomers (aSyn*). Statistical tests were performed using one-way ANOVA with repeated-measures for grouped analysis, followed by Tukey's post-hoc tests. Data were expressed as mean \pm SEM and a $0.5 \%$ general significance level was defined, with significance levels as follows: *: $\mathrm{p}<$ $0.05 ; * *: \mathrm{p}<0.01 ; * * *: \mathrm{p}<0.001$. Statistical significance is indicated with the symbol "\#" for the monomers, "+" for the dimers, and "**" for the summa between monomers and dimers. Scale bar: $30 \mu \mathrm{m}$ 


\subsubsection{Overexpression Of Rab 7 Reduces The Formation OF Dimers In CELls TREATED With ASyN WT}

Next, we investigated the fate of aSyn along the endocytic pathway by using Rab 7 as a marker.

H4 cells were then transiently transfected with Rab 7-GFP or with a dominant negative (DN) form of Rab 7 (Rab 7DN-GFP), treated with aSyn WT, aSyn A30P or aSyn A11P/V70P, and analysed by ICC and immunoblotting.

The overexpression of Rab 7 notably decrease the amount of internalized aSyn monomeric proteins, as well as the formation of dimers in $\mathrm{H} 4$ cells treated with aSyn WT, bringing the level of internalized aSyn WT close of the ones showed by H4 cells treated with aSyn A30P and with aSyn A11P/V70P (Figure 24A and 24B).

A possible explanation is that, by overexpressing Rab 7 and, therefore, the lysosomalrelated activity, the exogenously added and internalized aSyn got degraded faster and more efficiently, levelling out the differences previously reported and related to a more effective internalization of the wild-type form of the protein compared to the mutants, so to show finally a base level of internalization similar in all the three different conditions.

The use of the mutated, dominant negative form of Rab 7 further support this hypothesis, showing that when transfected with a Rab 7DN-GFP, the levels of internalization, the dimerization and the consequential inclusion formation abilities are rescued in $\mathrm{H} 4$ cells treated with aSyn WT, and that the overexpression of Rab 7DN does not affect the degradation of internalized aSyn monomeric proteins (Figure 24C and 24D). 
A $\quad$ NT

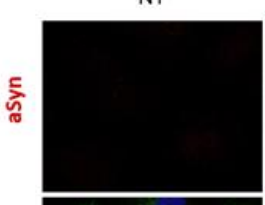

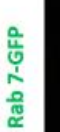

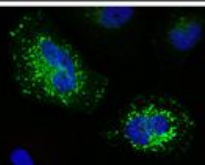

4
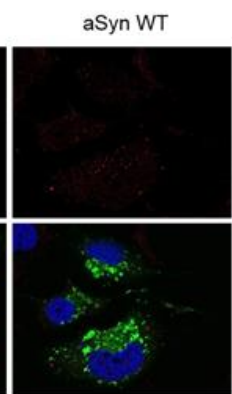

aSyn A30P

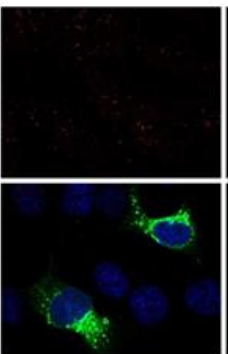

4

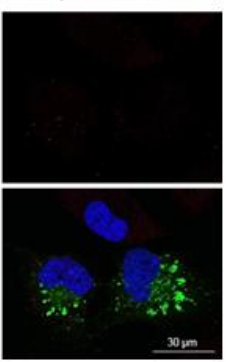

aSyn A11P/V70P

C

C NT

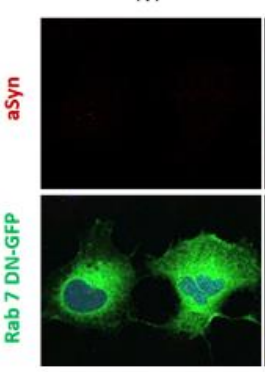

aSyn WT
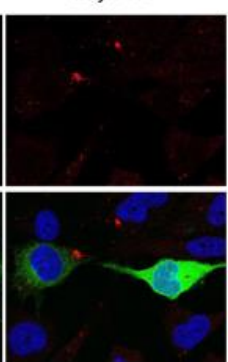

aSyn A30P
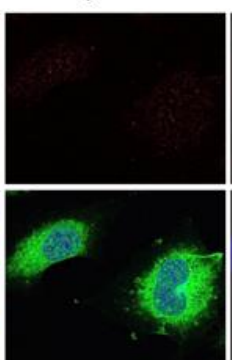

aSyn A11P/V70P
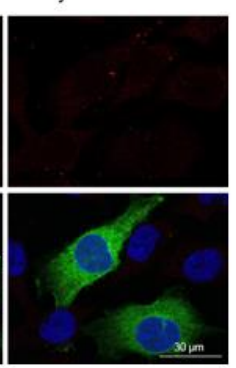

MW (kDa)

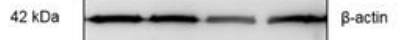

$35 \mathrm{kDa}$
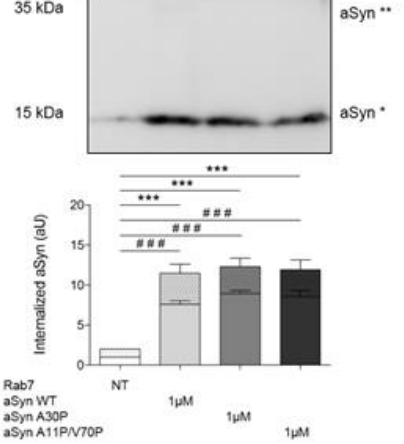

D

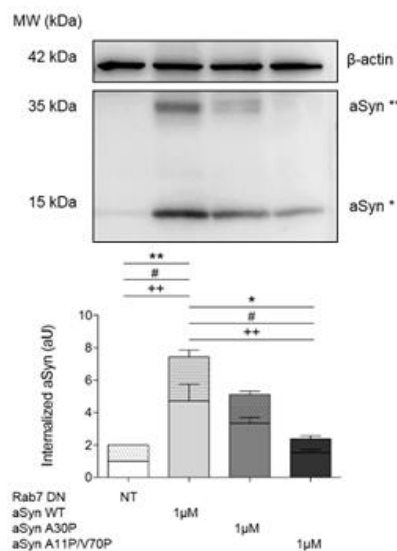

Figure 24. Rab 7 decreases the formation of dimers in $\mathrm{H} 4$ cells treated with aSyn WT monomers.

(A) ICC and (B) Immunoblotting of H4 cells transfected with Rab 7-GFP and treated as described above. (C and $\mathrm{D}$ ) The overexpression of the Rab 7 dominant negative (DN) does not affect the degradation of the internalized aSyn. (B and D, lower panel) Quantifications of the immunoblots. Dotted bars refer to the band corresponding to aSyn dimers $\left(\mathrm{aSyn}^{* *}\right)$, and clear bars refer to aSyn monomers (aSyn*). Statistical tests were performed using one-way ANOVA with repeated-measures for grouped analysis, followed by Tukey's post-hoc tests. Data were expressed as mean \pm SEM and a $0.5 \%$ general significance level was defined, with significance levels as follows: *: $p<0.05 ;{ }^{* *}: \mathrm{p}<0.01 ; * *$ : $\mathrm{p}<0.001$. The significance is showed with the symbol "\#" for the monomers, with the symbol "+" for the dimers and with the symbol "*" for the summa between monomers and dimers. Scale bar: $30 \mu \mathrm{m}$ 


\subsection{THE ENDOCYTIC PATHWAY AND THE ALP ARE INVOLVED IN THE PROCESSING OF ASYN}

\subsubsection{The Internalization Of ASyn Is MEDIATED By DyNAMIN}

We then investigated the endocytic mechanism involved in the internalization of aSyn by using two different blockers of endocytosis: PitStop2 (PitStop) and Dyngo 4A (Dyngo).

Pitstop is a novel, selective inhibitor of clathrin terminal domains that interferes with receptor-mediated endocytosis and impedes clathrin-mediated endocytosis (CME) by blocking the clathrin-coated pit dynamics at several different steps of the process ${ }^{231,232}$. Dyngo is a structural analogue of Dynasore with improved efficiency, and is an optimal candidate for the cure of various diseases (i.e., botulism) related to dynamindependent mechanism. Dyngo inhibits CME as well, but it was also reported that prevents SNAP25 cleavage, reduces synaptic vesicle endocytosis and activitydependent bulk endocytosis in cultured neurons and synaptosomes ${ }^{233}$.

For each chemical, we tested two different concentrations, based on what is reported in literature. For Pitstop, we used $30 \mu \mathrm{M}$ and $60 \mu \mathrm{M}$, and for Dyngo, $10 \mu \mathrm{M}$ and $50 \mu \mathrm{M}$. H4 cells, or H4 cells overexpressing Rab 4A-GFP were treated with the selected concentrations of the chemicals for 30 min before the treatment with aSyn monomers, then incubated for $24 \mathrm{~h}$ at $37^{\circ} \mathrm{C}$, following the same procedure and the same timing of the previous experiments. At the end of the treatment, WB and ICC analysis were performed in order to detect possible changes in the internalization rate.

In cells overexpressing Rab 4A, Dyngo effectively prevented the internalization and accumulation of aSyn in Rab 4A-surrounded vesicles. In contrast, PitStop failed to produce a significant effect (Figure 25A and 25B). Curiously, in $\mathrm{H} 4$ cells that do not overexpress Rab 4A, we confirmed the efficacy of Dyngo on blocking the internalization of aSyn. Instead, we observed the opposite effect using PitStop: from the quantification of the mean fluorescence intensity of the ICC (Figure 26B) and from the immunoblotting analysis (Figure 26C and 26D), we found that not only PitStop did not block the internalization of aSyn but it even stimulated it, increasing the accumulation of intracellular aSyn. 
A

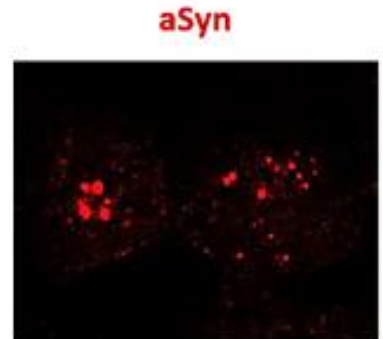

Rab 4A-GFP
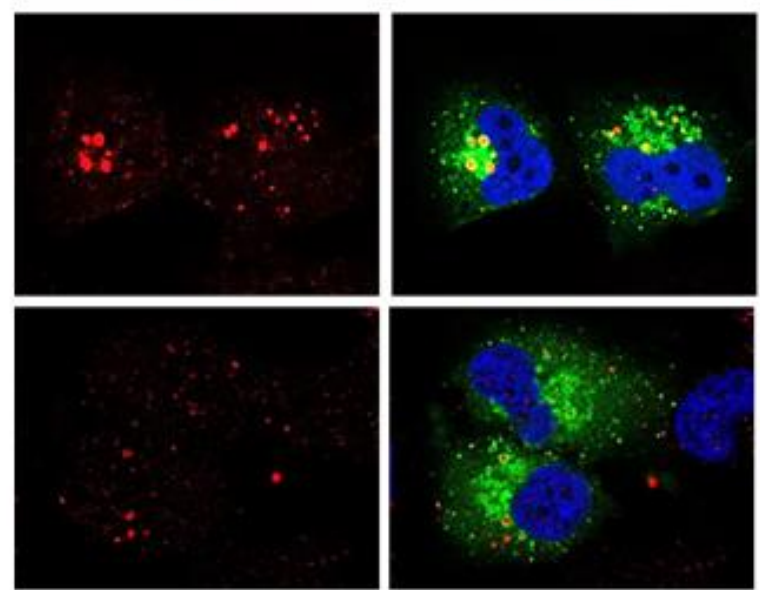

aSyn WT

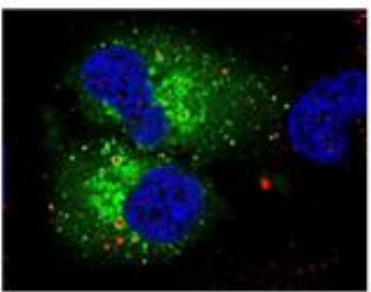

aSyn WT +

PitStop $30 \mu \mathrm{M}$
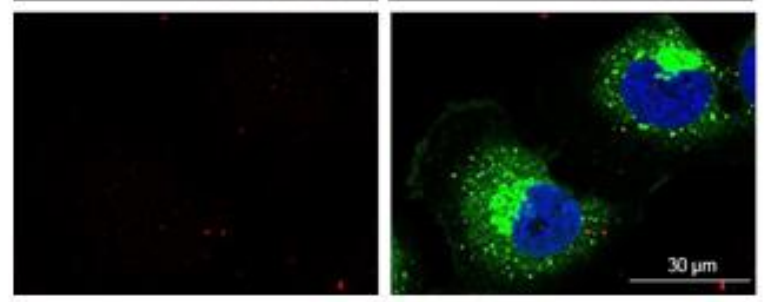

aSyn WT +

Dyngo $50 \mu \mathrm{M}$

B

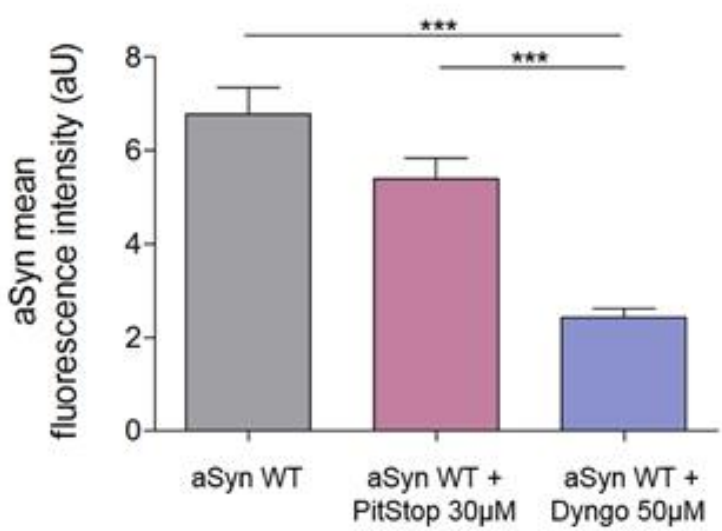

Figure 25. Dyngo effectively prevents the internalization of aSyn in $\mathrm{H} 4$ cells overexpressing Rab $4 \mathrm{~A}$, whereas PitStop does not have a significant effect.

(A) ICC on H4 Rab 4A-GFP cells, treated with 1uM aSyn monomers and with PitStop $30 \mu \mathrm{M}$ or with Dyngo $50 \mu \mathrm{M}$. Both of them are inhibitors of the endocytic processes. (B) Quantification of the aSyn mean fluorescence intensity in the three condition reported above. Scale bar: $30 \mu \mathrm{m}$

This could be related to a possible compensatory mechanisms: when clathrin-mediated process are blocked (PitStop- related effect), the clathrin-independent processes (such as synaptic vesicle endocytosis and activity-dependent bulk endocytosis) are exacerbated, and therefore, more aSyn is internalized. We can speculate that such a mechanism was mitigated by the overexpression of Rab 4A-GFP in the previous experiment, and therefore, was not possible to observe it so clearly. 
A
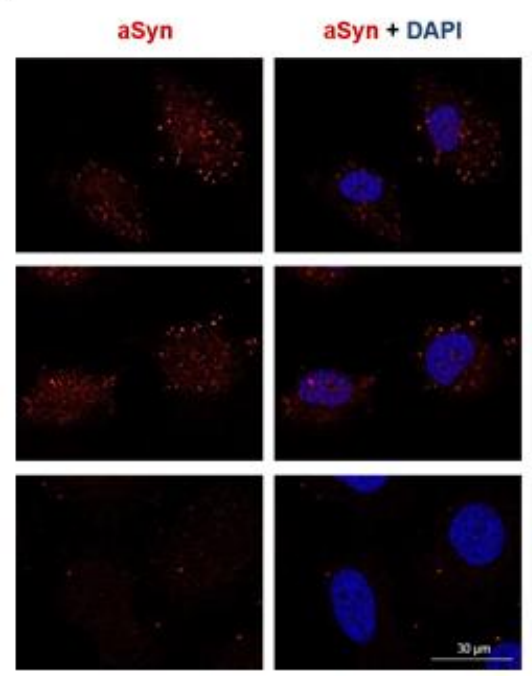

B

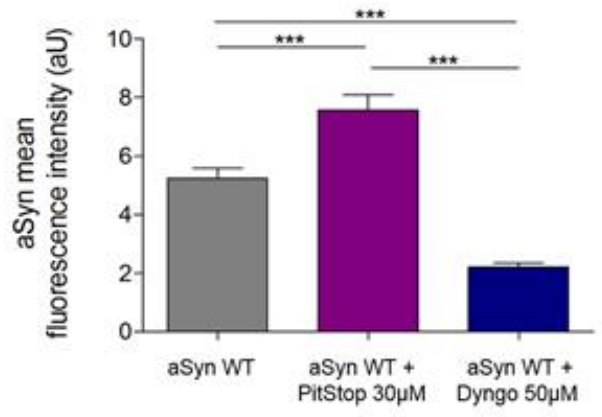

$\mathrm{C}$ MW (kDa)

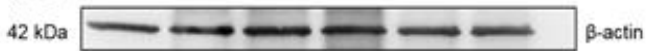

aSyn WT

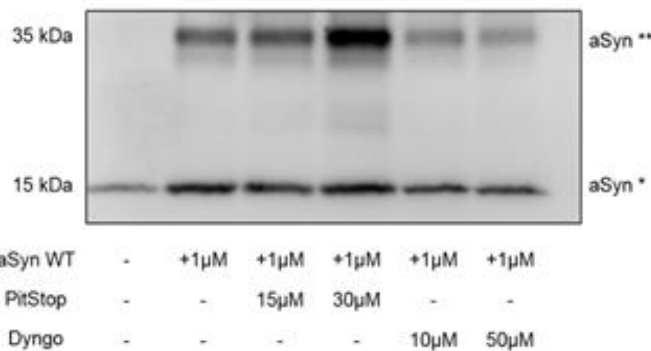

aSyn WT + Dyngo $50 \mu \mathrm{M}$

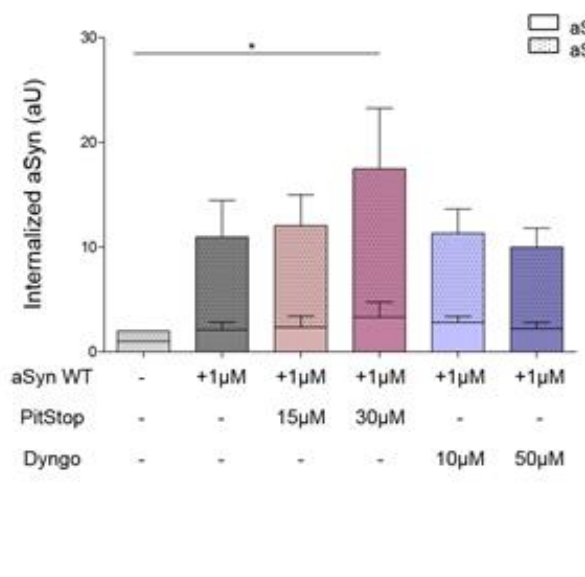

Figure 26. Dyngo effectively prevents the internalization of aSyn in $\mathrm{H} 4$ cells, whereas PitStop has the opposite effect.

(A) ICC of $\mathrm{H} 4$ cells treated with $1 \mu \mathrm{M}$ aSyn monomers and with vehicle, PitStop $30 \mu \mathrm{M}$ or with Dyngo $50 \mu \mathrm{M}$. Both PitStop and Dyngo are inhibitors of the endocytic processes. (B) Quantification of the aSyn mean fluorescence intensity in the three conditions. (C) Immunoblotting of $\mathrm{H} 4$ cells treated with different concentrations of PitStop and Dyngo. (D) Quantification of the immunoblot. Dotted bars refer to the band corresponding to aSyn dimers $\left(\mathrm{aSyn}^{* *}\right)$, and clear bars refer to aSyn monomers (aSyn*). Statistical tests were performed using one-way-analysis of variance (ANOVA), with repeated-measures for grouped analysis, followed by Tukey's post-hoc tests. Data were expressed as mean \pm SEM and a $0.5 \%$ general significance level was defined, with significance levels as follows: ${ }^{*}: \mathrm{p}<0.05 ; * *: \mathrm{p}<0.01 ;{ }^{* *}: \mathrm{p}<0.001$. The significance is showed with the symbol "\#" for the monomers, with the symbol "+" for the dimers and with the symbol "*" for the summa between monomers and dimers. Scale bar: $30 \mu \mathrm{m}$ 


\subsubsection{Impairment Of Autophagy Inhibits The Degradation OF ASYN}

Next, we tested whether an impairment of the ALP could have effect on the degradation of aSyn, to further explore the results obtained from the overexpression of $\mathrm{Rab}$ 7-GFP and Rab 7DN-GFP and their interaction with the internalized aSyn. For this, we used two different inhibitors of the autophagy/lysosome activity: Bafilomycin and Chloroquine.

Bafilomycin is an inhibitor of the late phase of autophagy. It prevents the maturation of autophagic vacuoles preventing the fusion between these and the lysosomes, due to the impairment of the vacuolar $\mathrm{H}+$ ATPase (V-ATPase). Chloroquine inhibits autophagy as well as it raises the lysosomal $\mathrm{pH}$ and this leads to inhibition of both fusion of autophagosome with lysosome and lysosomal protein degradation.

Also in this case, two different concentrations were tested for both the chemicals: for Bafilomycin 100nM and 200nM, while for Chloroquine 50 $\mu \mathrm{M}$ and $100 \mu \mathrm{M}$.

H4 cells, or H4 cells overexpressing Rab 7-GFP were treated with the selected working concentrations $30 \mathrm{~min}$ before the inoculation with aSyn monomers, then incubated for $24 \mathrm{~h}$ as previously described.

The ICC analysis and the corresponding quantification of the mean fluorescence intensity of aSyn showed that the impairment of the autophagy processes via both Bafilomycin and Chloroquine strongly inhibited the degradation of aSyn monomers in H4 cells transfected with Rab 7-GFP (Figure 27).

The same results are observed in H4 cells that do not overexpress Rab 7-GFP (Figure 28 , A to D).

It is worth to note that when treated with Chloroquine, the accumulation of aSyn was higher than that observed with Bafilomycin. This can be attribute to the fact that Chloroquine not only inhibits the fusion to the autophagosomes with lysosomes in the same way that Bafilomycin do, but also impedes the degradation of those proteins that are already accumulating within the lysosomes.

Taken together, these results suggest that aSyn internalized proteins are internalized via the endocytic pathway, processed and then degraded via the autophagy-lysosomal degradation system. 
A

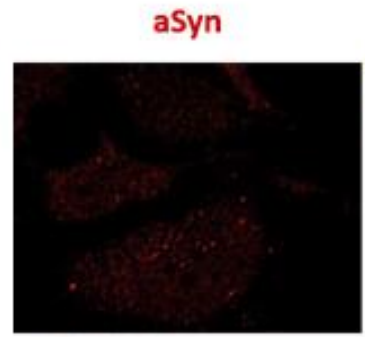

Rab 7-GFP
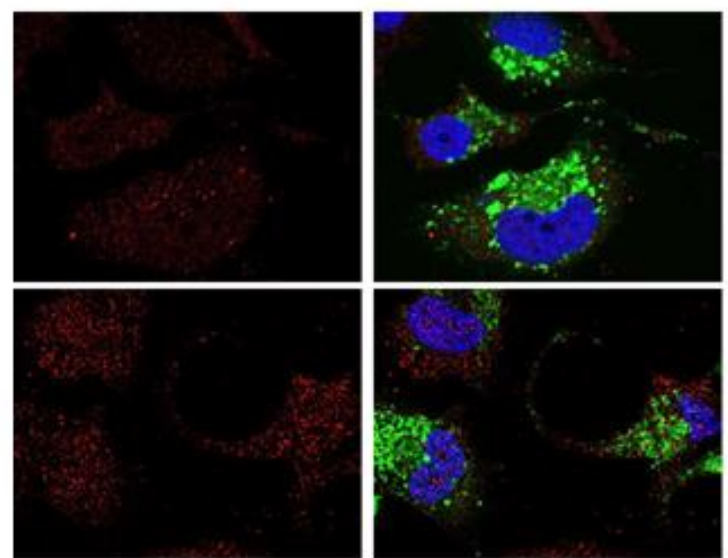

aSyn WT

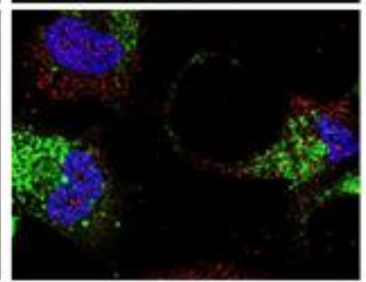

aSyn WT +

Baf $100 \mathrm{nM}$
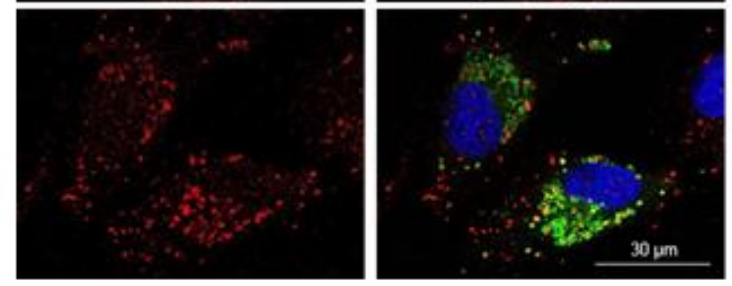

aSyn WT +

Chlq $50 \mu \mathrm{M}$

B

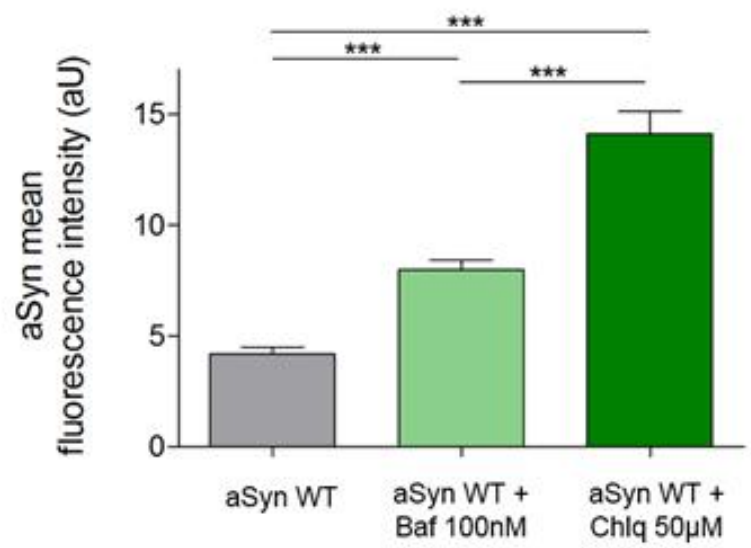

Figure 27. Impairment of autophagy inhibits degradation of aSyn in H4 cells transfected with Rab 7-GFP. (A) ICC of Rab 7-GFP-expressing cells treated with $1 \mu \mathrm{M}$ aSyn monomers and with vehicle, Bafilomycin 100nM (Baf $100 \mathrm{nM}$ ), or with Chloroquine $50 \mu \mathrm{M}(\mathrm{Chlq} 50 \mu \mathrm{M})$. Both of them are inhibitors of the ALP. (B) Quantification of the aSyn mean fluorescence intensity in the three condition reported above. Scale bar: $30 \mu \mathrm{m}$ 
A

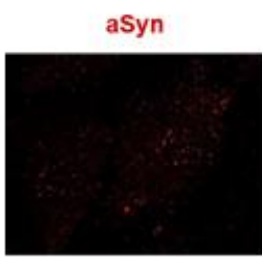

aSyn + DAPI
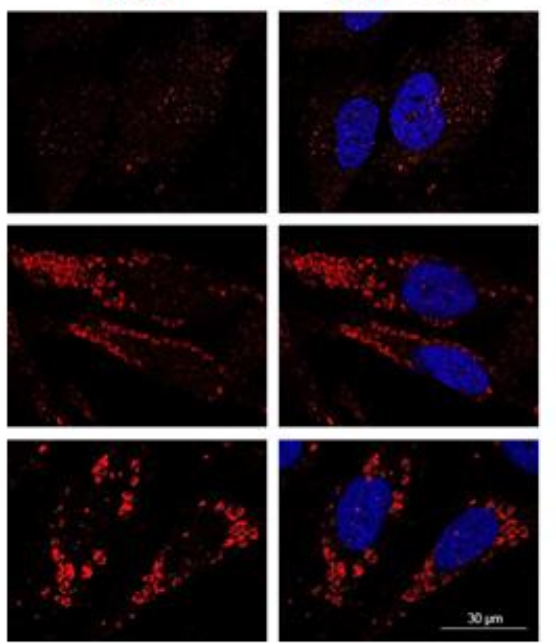

B

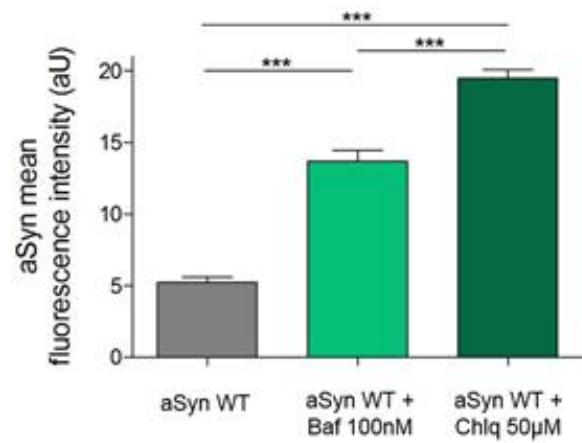

C

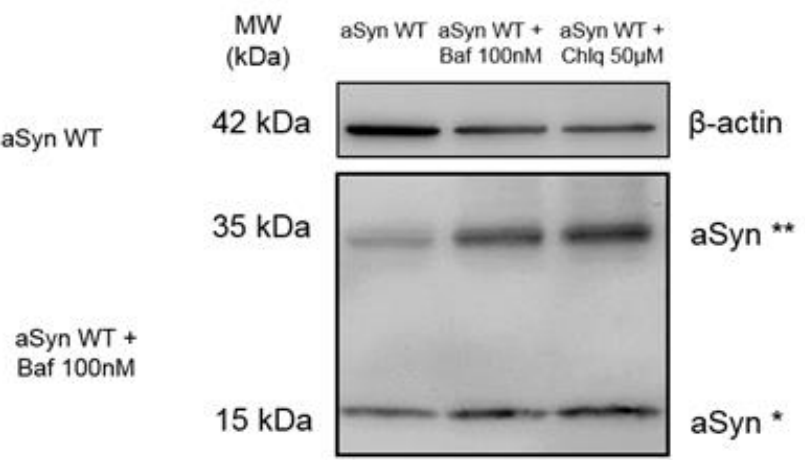

asyn WT +

Chlq $50 \mu \mathrm{M}$

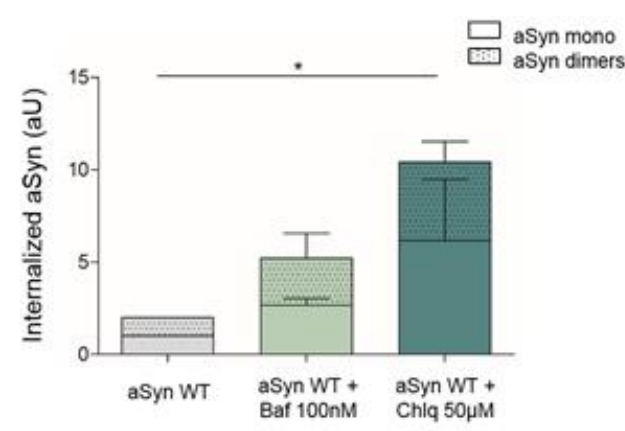

Figure 28. Impairment of autophagy inhibits the degradation of aSyn in $\mathrm{H} 4$ cells.

(A) ICC of $\mathrm{H} 4$ cells treated with $1 \mu \mathrm{M}$ aSyn monomers and with vehicle, Bafilomycin 100nM (Baf 100nM), or with Chloroquine $50 \mu \mathrm{M}$ (Chlq $50 \mu \mathrm{M})$. Bafilomycin and chloroquine are inhibitors of the ALP. (B) Quantification of the aSyn mean fluorescence intensity in the three condition. (C) Immunoblotting of H4 cells treated with $1 \mu \mathrm{M}$ aSyn WT and incubated with Bafilomycin $100 \mathrm{nM}$ or Chloroquine $50 \mu \mathrm{M}$. (D) quantification of the immunoblot in panel C. Dotted bars refer to the band corresponding to aSyn dimers (aSyn ${ }^{* *}$ ), and clear bars refer to aSyn monomers (aSyn*). Statistical tests were performed using one-way-analysis of variance (ANOVA), with repeated-measures for grouped analysis, followed by Tukey's post-hoc tests.Data were expressed as mean \pm SEM and a $0.5 \%$ general significance level was defined, with significance levels as follows: $*: \mathrm{p}<0.05 ; * *: \mathrm{p}<0.01 ; * *: \mathrm{p}<0.001$. The significance is showed with the symbol "\#" for the monomers, with the symbol "+" for the dimers and with the symbol "*" for the summa between monomers and dimers. Scale bar: $30 \mu \mathrm{m}$ 


\subsection{BIOPHYSICAL CHARACTERIZATION OF SYNT}

The mechanisms triggering aSyn aggregation are still not clear, nor the precise relationship between aSyn aggregation and neurotoxicity. Moreover, aSyn aggregates are polymorphic, ranging from soluble oligomeric forms, to ring- or string-like oligomers, spherical-shaped protofibrils, and insoluble fibrils. Currently, oligomeric forms of aSyn are hypothesized to be the most cytotoxic forms of the protein, rather than mature fibrillar aggregates. In this section of the results we report the results obtained from the structural characterization of a C-terminal modified form of aSyn, SynT, which renders aSyn more prone to aggregation in cell models.

\subsubsection{C-TERMINALLy MODIFIED ASYN FormS INCLUSIONS IN H4 CELLS}

As previously described, truncations and C-terminal modifications of aSyn alter its propensity to aggregate. In particular, expression of SynT in $\mathrm{H} 4$ cells resulted in the formation of cytosolic inclusions that exhibit LB-like properties (Figure 29A-B) ${ }^{208}$. Inclusion formation is potentiated by the co-expression of SynT together with Synphilin-1, an interactor of aSyn also present in LBs and LNs, as extensively described by several groups ${ }^{213,214,234}$. In contrast, co-expression of untagged aSyn with Synphilin-1 did not form discrete inclusions (Figure 29B), suggesting that modifications in the $\mathrm{C}$-terminal region alters the aggregation properties of aSyn. 
A

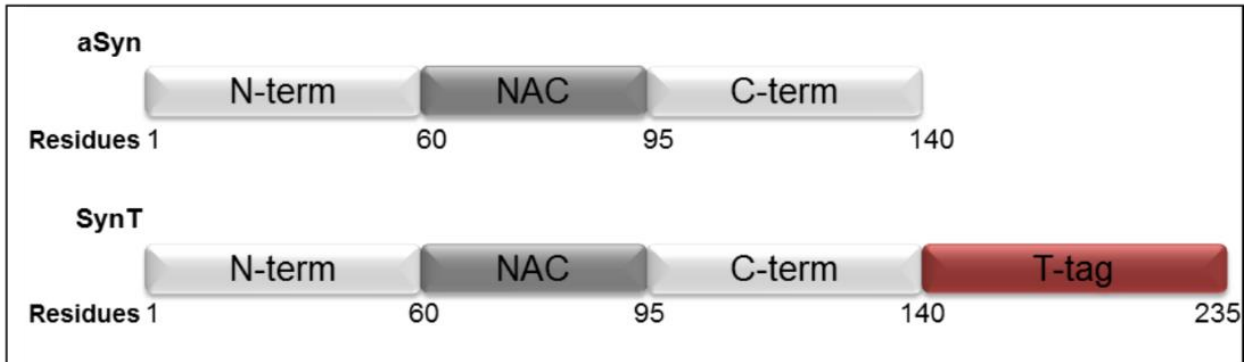

$\mathrm{B}$

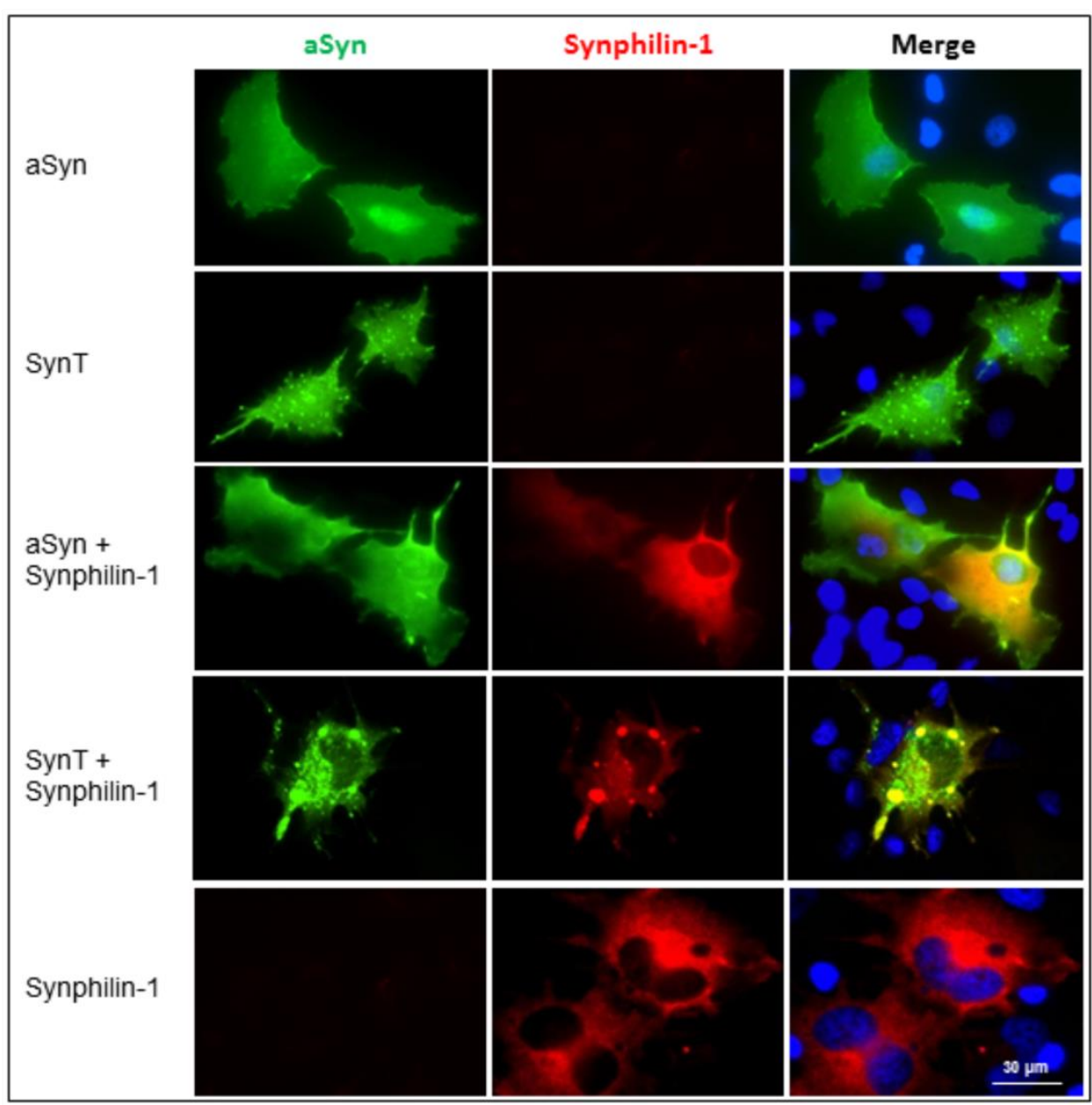

Figure 29. C-terminal modified aSyn (SynT) forms inclusions in $\mathrm{H} 4$ cells.

(A) Schematic representation of the structure of human aSyn highlighting the N-terminal (N-term), NAC (NAC) and C-terminal (C-term) regions of the protein. Amino acid residue numbers are indicated for aSyn and for SynT. (B) aSyn distribution in cells transfected with aSyn, SynT and SynT/Synphilin-1. In cells transfected with aSyn, or with aSyn and Synphilin-1, aSyn is diffusely distributed throughout the cell. In contrast, in cells transfected with SynT, or with SynT and Synphilin-1, aSyn forms inclusions of various sizes and morphologies. 


\subsubsection{The Intrinsically Disordered Nature of ASyn Is Not INFLUENCED By The C-TERMinAl ModificATION IN SyNT}

To analyse the impact that the C-terminal modification in SynT might have on the structural features of aSyn, we employed NMR spectroscopy to assess structural features of recombinant aSyn and SynT proteins.

To investigate the molecular dynamics of SynT we produced N-terminally acetylated forms of recombinant aSyn and SynT, which we used for in vitro studies.

Prior to NMR measurements, we analysed the recombinant SynT by SDS-PAGE and by gel-filtration chromatography (Figure 30A and 30B), confirming that the modified protein was efficiently expressed and purified from the soluble fraction. First, a twodimensional ${ }^{1} \mathrm{H}^{-15} \mathrm{~N}$ heteronuclear single quantum coherence (HSQC) spectrum of a $100 \mu \mathrm{M}$ sample of uniformly ${ }^{15} \mathrm{~N}$-labeled SynT in buffer B (MES $20 \mathrm{mM}, \mathrm{NaCl} 100$ $\mathrm{mM} \mathrm{pH} \mathrm{6.5)} \mathrm{was} \mathrm{recorded} \mathrm{at} 15^{\circ} \mathrm{C}$ (Figure 31A). The NMR spectrum of SynT retains the excellent resolution of the unmodified aSyn native protein. The resonances are defined and well resolved, with a limited dispersion of chemical shifts that reflect the unfolded, disordered nature of the protein and the mobility's degree of the backbone. The overlaid spectra of SynT and aSyn demonstrate that the SynT protein remains intrinsically disordered upon the C-terminal modification with the EGFP fragment (Figure 31A). Notably, although backbone chemical shift changes were observed in a discrete number of residues located in the vicinity of the EGFP fragment at the Cterminal region of the protein, small but measurable chemical shift changes became also evident in the region of amino acids 1-10, far from the C-terminus (Figure 31B). Finally, we observed that only a small number of new resonances appeared in the SynT construct compared to aSyn, likely belonging to the first residues of the EGFP fragment fused to aSyn. The rest of the signals corresponding to the EGFP fragment are likely broadened beyond detection. To gain insight into the secondary structure of the EGFP fragment, we registered the far-UV CD spectra of aSyn and SynT (Figure 31C). While the spectrum of the untagged protein clearly reflected the intrinsically disorder nature of the protein, we found that the SynT presented a substantial change in the CD spectra. In addition to the deep minimum at $\sim 198 \mathrm{~nm}$, a minimum around $220 \mathrm{~nm}$ appeared, which is characteristic of $\beta$-sheet secondary structural elements (Figure 31C). In fact, when subtracting the random coil component measured for aSyn in the same conditions, we observed a pure $\beta$-sheet signature, likely reflecting the conformational features of the EGFP truncated tag, which is expected to be rich in $\beta$ barrel elements according to the 3D structure of full-length $\mathrm{EGFP}^{235}$. 
A

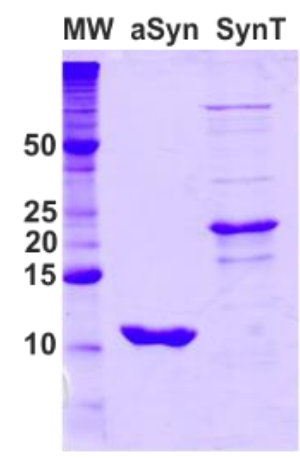

B

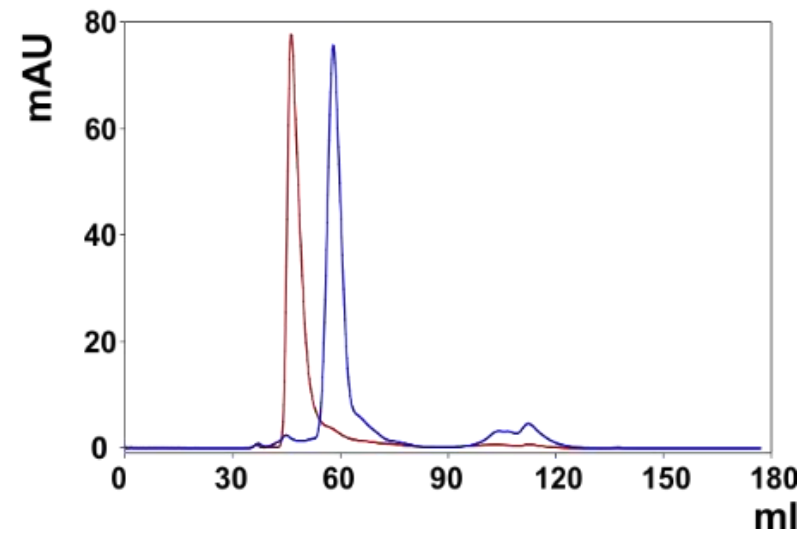

Figure 30. Biophysical characterization of SynT.

(A) Comassie staining of purified aSyn and SynT loaded in PAGE-SDS 12\%. (B) Size

Exclusion Chromatography profiles of aSyn (blue) and SynT (red). 


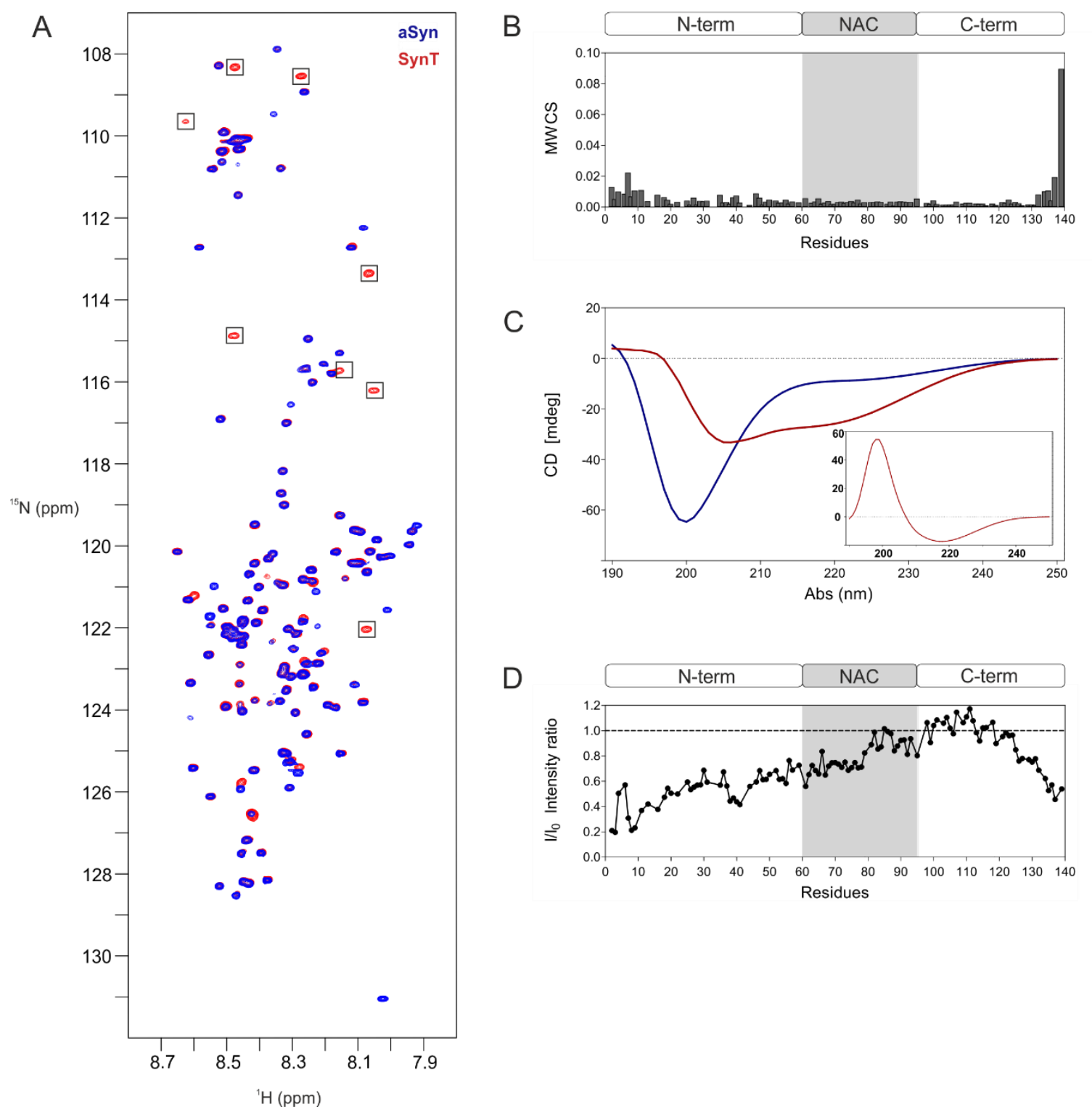

Figure 31. NMR characterization of SynT.

(A) Overlaid $2 \mathrm{D}^{1} \mathrm{H}-{ }^{15} \mathrm{~N}$ HSQC NMR spectra of aSyn $(100 \mu \mathrm{M})$ in blue and SynT $(100 \mu \mathrm{M})$ in red. Boxed crosspeaks indicate additional SynT signals, most likely from the flexible linker region and the first amino acids of the tag. (B) Mean weighted ${ }^{1} \mathrm{H}^{-15} \mathrm{~N}$ chemical shift perturbation plot of SynT versus aSyn. (C) CD spectra of aSyn (blue) and SynT (red). Inset shows the SynT-aSyn difference spectra featuring the $\beta$-sheet rich spectra for the tag. All NMR experiments were recorded at $15^{\circ} \mathrm{C}$ using aSyn and SynT samples dissolved in buffer B supplemented with $10 \% \mathrm{D}_{2} \mathrm{O}$. (D) SynT/aSyn NMR signal intensity ratios. Signal attenuation was observed at the $\mathrm{N}$-terminus and the $\mathrm{C}$-terminal residues that are proximal to the EGFP fragment 


\subsubsection{Transient Interactions Between The N- And The C- Terminal Regions Are Strengthened In SynT}

The anchoring of the truncated EGFP segment at the C-terminus of aSyn in SynT resulted in chemical shift changes for resonances in the vicinity of the C-terminus (Figure 31B). In addition, chemical shift changes were detected for a discrete number of residues located at the $\mathrm{N}$-terminus. To obtain further information about a potential role of the N-terminus of SynT, we analysed the ${ }^{1} \mathrm{H}-{ }^{15} \mathrm{~N}$ HSQC intensity profile of SynT and compared it to that of aSyn, which is not C-terminally extended by the EGFP fragment (Figure 31D). The comparison showed that NMR signal intensities are decreased most strongly at the N-terminus of SynT and steadily increase up to and through the NAC region until values similar to aSyn are found for residues 80-120. The steady increase is interrupted for residues 38-41, next to the aromatic residue Tyr39. Beyond residue 120, NMR signal intensities strongly decreased towards the attached EGFP fragment, most likely due to an increase in rotational correlation time caused by the EGFP fragment.

These observations led us to evaluate SynT conformation and its dynamic properties. First, we measured an almost complete set of ${ }^{3} J_{\mathrm{HN}-\mathrm{H \alpha}}$ couplings in aSyn and SynT states, in order to have quantitative and reliable information of the time-averaged distribution of the backbone torsion angles $\phi^{219,236}$. As shown in Figure 32A, the values measured for the two forms of the protein are essentially indistinguishable, indicating that the $\mathrm{C}$ terminal modification with the EGFP fragment in the SynT form did not influence the local conformational propensities adopted by untagged aSyn.

Next, we evaluated the molecular dynamics properties of SynT. To this end, we measured ${ }^{15} \mathrm{~N} R_{1}$ and $R_{2}$ spin relaxation rates and heteronuclear $\left({ }^{1} \mathrm{H}-{ }^{15} \mathrm{~N}\right)$ steady-state NOEs (hetNOEs), which are sensitive to protein motions on multiple time scales. This set of experiments was first performed on untagged aSyn at a ${ }^{1} \mathrm{H}$ spectrometer frequency of $600 \mathrm{MHz}$. In agreement with previous results ${ }^{237}, R_{1}$ values ranged between 1.3 and $1.7 \mathrm{~s}^{-1}, R_{2}$ values between 2.0 and $3.5 \mathrm{~s}^{-1}$, and hetNOEs in the range 0 to -0.3 (Figure 32B, 32C and 32D), with lower values observed at the termini and in the NAC region. The attachment of the EGFP fragment to aSyn resulted in no significant changes in the $R_{1}$ and hetNOEs values, with the exception of the very terminal residues. However, pronounced deviations were observed for the $\mathrm{R} 2$ relaxation rates (Figure 32C). Consistent with the profile of intensity ratios (Figure 31D), R2 rates were increased for the $\mathrm{N}$-terminal half of the aSyn sequence, when the EGFP fragment was attached at the C-terminus, which also experienced increased transverse relaxation. 
Whereas increased $\mathrm{R} 2$ rates at the C-terminus can be connected to slower rotational tumbling of the $\beta$-structure containing EGFP fragment (Figure 31D), the faster transverse relaxation in the $\mathrm{N}$-terminal half of the aSyn sequence points to an exchange process driven by transient interactions between the $\mathrm{N}$-terminal region of aSyn and the EGFP fragment. Notably, Tyr39 had higher R2 rates than its surrounding residues, suggesting contributions of aromatic interactions in the exchange process.

\subsubsection{The N-Terminal Region Of Synt Is Less Available THAN IN ASYN}

To further investigate the influence of the EGFP fragment on the $\mathrm{N}$-terminal region of aSyn, we compared the availability of this region for binding known small molecules. To address this, we used the phthalocyanine tetrasulfonate (PcTS) molecule as a structural probe ${ }^{238}$, since its binding preference for the $\mathrm{N}$-terminal region results from a balance between the presence of aromatic side chains in that region and the degree of interaction with the negatively charged C-terminal region. Although the binding of PcTS at the N-terminus of aSyn was evident already at 0.25 equivalents of the compound, no broadening or chemical shift perturbations were observed under similar conditions, nor at higher concentrations, such as 0,5 or 1 equivalent, for the amide groups of residues located in the N-terminus of SynT (Figure 33A). The absence of PcTS interaction with the N-terminus of SynT construct could be caused by an increased binding of PcTS to the EGFP fragment compared to the N-terminus of SynT. Alternatively, intra- or intermolecular interactions between the EGFP fragment and the N-terminus of SynT could interfere with PcTS binding. For example, interaction of the EGFP fragment with the N-terminus of SynT would bring the highly negatively charged aSyn residues $100-140$ in close proximity to the $\mathrm{N}$-terminal region, possibly resulting in electrostatic repulsions between the negatively charged C-terminal region and peripheral sulfonate groups in the ligand.

Finally, we tested whether the EGFP fragment in SynT interfered with binding and folding of aSyn on the surface of SUVs, a process that is mainly driven by the $\mathrm{N}$ terminal region. To address this, we generated SUVs from a 5:3:12 mixture of DOPE:DOPS:DOPC. NMR spectroscopy revealed that aSyn interacted with DOPE:DOPS:DOPC liposomes (Figure 33B) as previously described ${ }^{229}{ }^{230}$. In contrast to untagged aSyn, the EGFP fragment in SynT influenced the residue-specific SUVinduced broadening of the NMR signals. Less broadening was induced at the N- 
terminus of SynT, potentially due to its decreased availability, suggested both by $15 \mathrm{~N}$ spin relaxation measurements and PcTS interaction studies (see above). At the same time, residues up to 130 of SynT experienced signal attenuation in the presence of SUVs, while the C-terminal residues of untagged aSyn only contribute little to the interaction with membranes ${ }^{154}$.

\subsubsection{SynT DOEs Not Form AMYloId FibriLs IN VITRO}

Next, we investigated whether the EGFP fragment affected the fibrillization of aSyn in vitro, since it affects the aggregation of the protein in cultured cells. Recombinant aSyn or SynT were incubated at $37^{\circ} \mathrm{C}$, under constant agitation, and fibrillization kinetics was monitored by Thio-T binding. Surprisingly, SynT did not show any fibrilspecific increase in Thio-T intensity in vitro (Figure 33C).

Amyloid fibril formation was further assessed by TEM analysis on negatively stained samples of aSyn and of SynT, after the aggregation protocol. Consistently with the Thio-T assay, we found that aSyn formed well-defined amyloid filaments (Figure 33D). In contrast, SynT formed non-fibrillar, amorphous aggregates. 
A

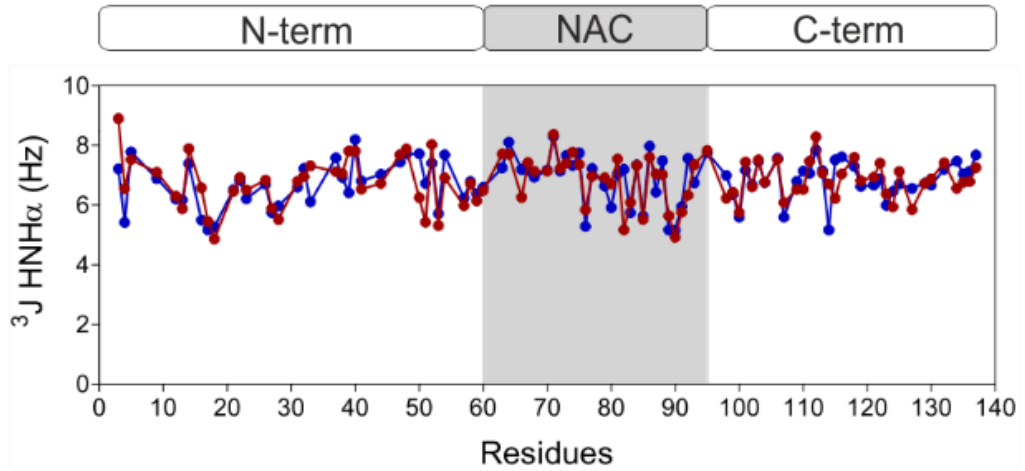

B
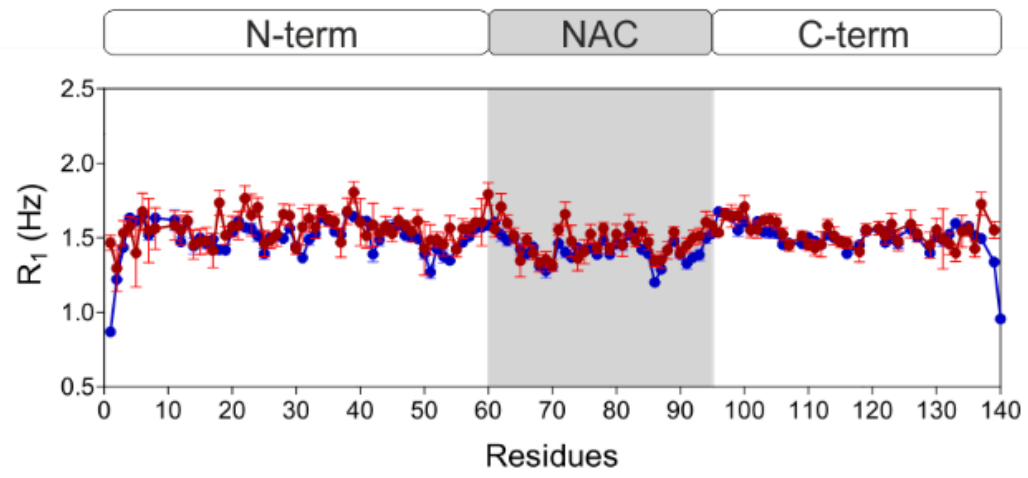

C

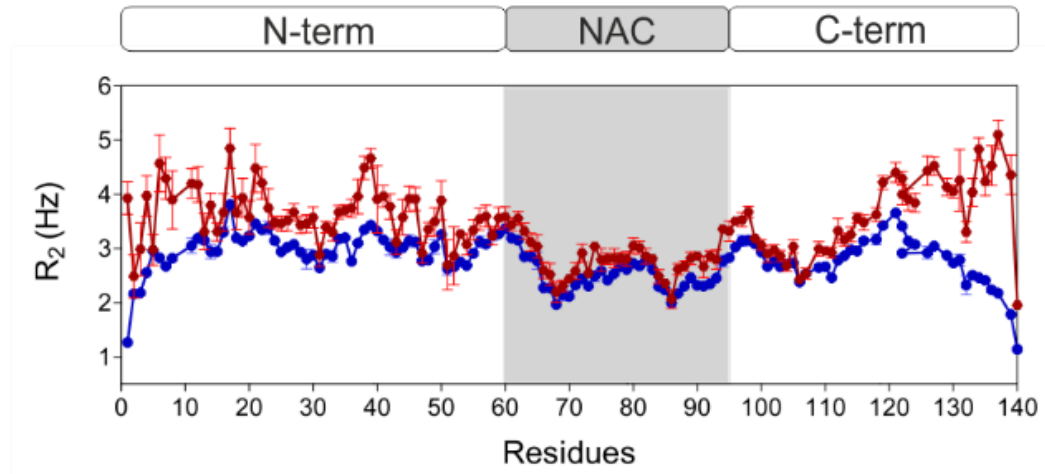

$\mathrm{D}$

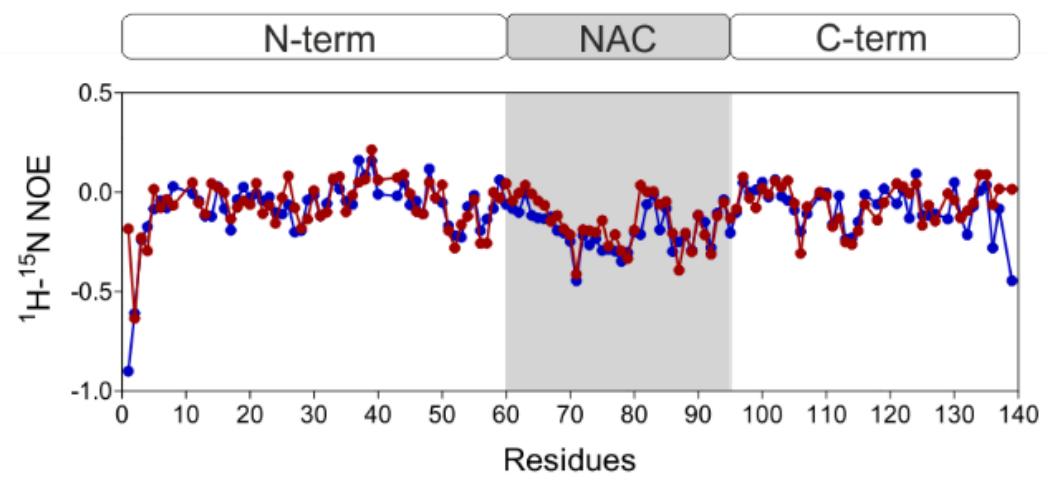

Figure 32. Backbone dynamics of SynT.

(A) ${ }^{3} \mathrm{~J} \mathrm{HN}-\mathrm{H} \alpha$ coupling constants measured for aSyn (blue) and SynT (red). (B,C) ${ }^{15} \mathrm{~N}$ relaxation rates of aSyn (blue) and SynT (red). Panels (B) and (C) depict $\mathrm{R}_{1}$ and $\mathrm{R}_{2}$ rates, respectively. Panel (D) shows ${ }^{1} \mathrm{H}-{ }^{15} \mathrm{~N} h N O E$ profiles for both proteins. All experiments were recorded at $15^{\circ} \mathrm{C}$ using aSyn $(100 \mu \mathrm{M})$ and SynT $(90 \mu \mathrm{M})$ dissolved in buffer B supplemented with $10 \% \mathrm{D}_{2} \mathrm{O}$. 
A

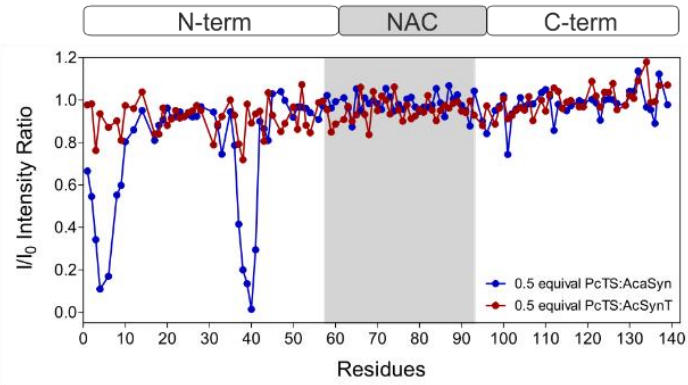

C

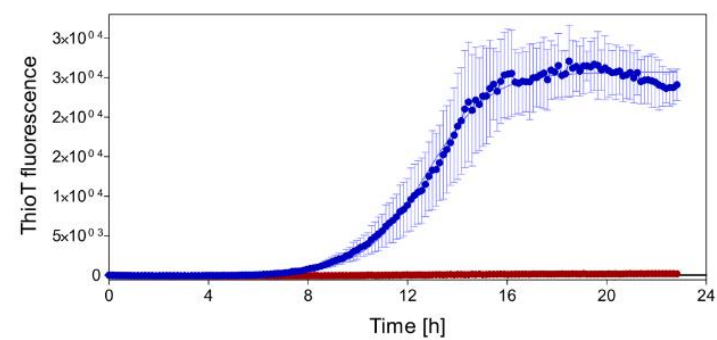

$\mathrm{B}$

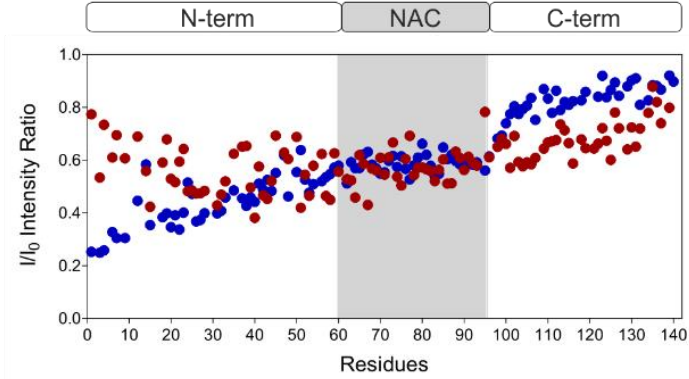

D

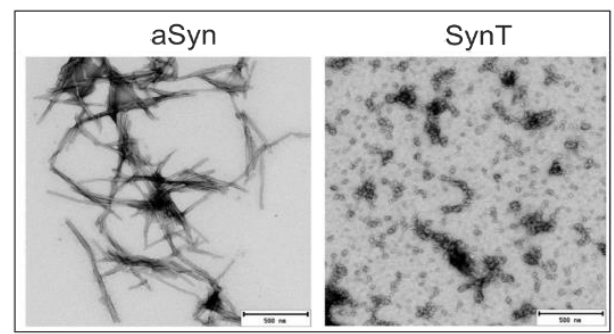

Figure 33. Binding and aggregation properties of SynT.

Interaction profiles of the backbone amide groups of $90 \mu \mathrm{M}$ aSyn, in blue and $90 \mu \mathrm{M}$ SynT proteins, in red, in the presence of 0.5 molar equivalent of PcTS (A). Membrane binding properties of aSyn (Blue) and SynT (red) in the presence of artificial small unilamellar vesicles membranes (SUVs) [1:100 protein:SUVs ratio] (B). Continuous ThioT aggregation assays of a $25 \mu \mathrm{M}$ solutions of aSyn (blue) and SynT (red). The proteins were diluted in Buffer A (25mM Tris, $\mathrm{pH}$ 7.7) (C). TEM images of the resulting aggregates stained with uranyl acetate (scale bar, $500 \mathrm{~nm}$ ) (D). 


\section{- V - \\ DiscUsSION}




\section{DisCUSSION}

A key premise for the prion-like spreading hypothesis of $\mathrm{PD}$ and other synucleinopathies is that aSyn assemblies will be taken up by a recipient cell, transported and undergo modifications within the cell, further seeds aggregation by misfolding the endogenous, otherwise healthy proteins and finally transferred to another cells and propagate the pathology. Although there are compelling evidences supporting this hypothesis, is still unclear how this process occurs in PD patients and where and how aSyn aggregation initiates.

In this study, we focused on the very first steps of this complex series of events, and we tried to understand how important the interactions with plasma membrane and with the trafficking machinery pathways are during the internalization and the processing of exogenously added aSyn.

We examined the internalization of different species of aSyn (in the form of monomers and fibrils) via ICC and immunoblot analysis, and we found that aSyn monomers enter cultured cells and form perinuclear, punctuated inclusions, possibly composed of high molecular weight species.

Afterwards, we studied the interplay of aSyn with several proteins involved in different steps of the trafficking machinery pathway, by screening a family of small GTPases collectively known as RABs. In order to achieve this, we transiently transfected our cultured cells with a library of EGFP-tagged RABs and we treated them with aSyn monomers and fibrils. Then we screened these for morphological changes and colocalization between RABs and aSyn.

We found that aSyn inclusions formed upon the internalization of aSyn monomers partially colocalize with Rab $5 \mathrm{~A}$ and Rab 7, suggesting that the endocytic pathway and the lysosomal one are involved in the processing of internalized aSyn. We also found that the overexpression of Rab $4 \mathrm{~A}$ alter the size of aSyn inclusions, inducing a considerable increase in the rate of internalized protein. To note, the morphology of $\mathrm{Rab} 4 \mathrm{~A}$ positive vesicles undergoes significant modifications as well, suggesting that aSyn internalization disturbs Rab 4A function and that the protein itself may play an important role in aSyn uptake.

In parallel, we analysed more in depth the role of aSyn membrane binding properties and its importance in the internalization and in the interaction with the endocytic and the lysosomal pathway. To do this, we used three different mutant that progressively lose the ability to bind to membranes. 
Based on the results obtained, we confirmed that both aSyn monomers and fibrils need to temporarily interact and bind to the plasma membrane during the internalization process, and that even a partial deficiency in the membrane binding capacities it has a remarkable effect on the ability of aSyn monomers to get internalized in cultured cells. Finally, by using selective inhibitors of the endocytic and lysosomal pathways, we could demonstrate that aSyn is internalized via a dynamin-dependent process, but not throughout CME. We also could prove the involvement of the ALP as a selected mechanism in the intracellular degradation process of aSyn

In the second part of the study, we went forward in the analysis of aSyn pathological processes and our focus moved on the dissection of the biophysical properties of an aggregation-prone form of aSyn, SynT.

Using NMR we performed a detailed structural characterization of SynT through a systematic comparison with the unmodified aSyn. We found that the conformations adopted by SynT resemble those described for the unmodified protein and that the intrinsically disordered nature of aSyn is not strongly influenced by the C-terminal modification in SynT. Nevertheless, a few differences were observed at the N-terminal region, and the transient interactions between the $\mathrm{N}$-terminal and the $\mathrm{C}$-terminal regions in SynT resulted strengthened if compared to aSyn.

The use of small molecules as a structural probe (such as PcTS) as well as the membrane-binding assay performed helped us to further demonstrate that the $\mathrm{N}$ terminal region of SynT is less available than in aSyn, suggesting that disturbances in the N-terminal region and a consequent decrease of membrane-binding properties might contribute to the pathobiology of aSyn.

\subsection{ASyn MEMbrane Binding Properties Are ESSENTIAL FOR ITS INTERNALIZATION}

While the normal function of aSyn and the mechanisms related to its pathological behaviour remains uncertain, it is clear that the interaction of aSyn with membranes plays a crucial role in both aSyn biology and pathology.

The conformational changes acquired by the protein - mainly related to the $\mathrm{N}$-terminal lipid binding domain - during the transition from a free state to a membrane-bound one, are considered functionally important and have been described in details $\mathrm{s}^{72}$. Recently a detailed, structural characterization of the three distinct regions of aSyn - 
each one possessing different dynamical behaviour and membrane-bound affinities has been provided ${ }^{154}$.

During the internalization process, oligomeric or protofibrillar aSyn species have been reported to permeabilize membranes by acting as a pore or channel ${ }^{116,239}$. One or more molecules of monomeric aSyn, too, may at times acquire a more structured conformation and cluster together to form membrane permeabilizing channels ${ }^{121}$, although contrasting evidences suggest that they could rather passively diffuse throughout membranes ${ }^{129,240}$.

The present study revealed that both aSyn monomeric and fibrillary species are internalized in cultured cells via an active process that requires, in the very first step, a transient interaction with the plasma membrane, as clearly shown from the biotinylation assay. The data obtained repeatedly confirmed as well that the membrane binding properties of aSyn are extremely important, if not essential, for its internalization and for the resulting interaction with the endocytic pathway, the transition from one cellular compartment to another (from the early endosome to the late endosome and therefore, to the lysosome) and finally for its degradation.

The use of different mutant together with aSyn WT, such as the PD familial mutant $\mathrm{A} 30 \mathrm{P}$ and the artificial one A11P/V70P, which show a progressive lack of the membrane binding abilities, allowed us to characterize more in details the importance of these properties in relation to the uptake, the processing and the inclusion formation. Based on the trend observed in the experiments performed, we can clearly state that to a greater deficiency of the membrane binding properties correspond a greater reduction in the rate of internalization.

This could also be explained in light of the observation of the three different regions in aSyn and their diverse membrane-bound behaviour as recently hypothesized ${ }^{154}$ (Figure 10). In fact, the A30P mutation affects the so called "intermediate" central region of aSyn (residues 25 to 98), which was suggested to play a role in modulating the affinity of aSyn for different type of membranes.

Structurally, the substitution of an alanine with a proline itself play a fundamental role in the disruption of the membrane-bound $\alpha$-helical formation. Thus, an impairment in this region would most likely affect the strength by which the protein interact and bind to membranes, exerting a bigger or minor effect accordingly to the curvature of the membrane or to the lipid composition. That is the reason why we observe a consistent decrease in the internalization of aSyn A30P compared to aSyn WT. 
On the other side, the double point mutations in aSyn A11P/V70P affect both the Nterminal region (residues 1 to 25), that is rigidly bound and act as a membrane anchor, and the central region (residues 25 to 98 ).

In this case, the substitution of the alanine in position 11 and the valine in position 70 with two prolines disrupts completely both the ability of the mutant to anchor, in a first place, to the membrane with its $\mathrm{N}$-terminal region, and, in a second moment, to further modulate the binding via its central region. To confirm the previous hypotheses, the membrane-binding assay performed show as, starting from the residue 11, the ability of the protein aSyn A11P/V70P to bind to the membranes is completely compromised (Figure 24A).

Thus, although we can observe a consistent decrease in the internalization of aSyn A30P compared to aSyn WT, the uptake of this mutant is still bigger than the one related to aSyn A11P/V70P.

Taken together, these results highlight the extreme importance of the membrane binding properties during the first step of the internalization of aSyn.

\subsection{The RABs Screening And Rab 4A: An Important Player In ASyn PATHOLOGY?}

The RABs are a family of small GTPases that organize the shapes and the dynamics of intracellular membranes in all the eukaryotic cells, by regulating the trafficking machinery pathway ${ }^{241}$. It is therefore not surprising that mutations and dysregulation of RABs and their effectors are implicated in several human pathologies, ranging from hereditary disorders to cancer and to neurological and neurodevelopmental disorders ${ }^{178,241}$. As mentioned before, several RABs have indeed been directly or indirectly linked to $\mathrm{PD}$ and synucleinopathies and it is widely recognised that membrane trafficking defects play a key role in the development of the pathology.

In this study, we performed a comprehensive screen of the RABs members and later on, we analysed in greater details a pool of selected RABs that were showing changes in the morphology or a direct interaction with aSyn.

Among the $>60$ members analysed, we found stronger effects with Rab 4A, Rab 5A and Rab 7 (and to a minor extent with Rab 9A).

$\mathrm{Rab} 4 \mathrm{~A}$ and Rab 5A are localized in the early endosome, contributing to the protein recycling/transport to the plasma membrane and early endosome fusion, respectively, 
while Rab 7 is localized in the late endosome, lysosome and phagosome, contributing to the late endosome to lysosome fusion process.

Our data clearly show that aSyn partially colocalize with Rab 5A and Rab 7, suggesting that the endocytic pathway first and the ALP later are involved in the internalization, processing and degradation of exogenously added aSyn monomers.

However, the most interesting result came from the analysis of Rab $4 \mathrm{~A}$.

$\mathrm{H} 4$ cells overexpressing Rab 4A showed a greater internalization of aSyn monomers, which accumulate in bigger inclusion surrounded by $\mathrm{Rab} 4 \mathrm{~A}$ positive vesicles. On the same time, a dramatic increase in the size of Rab $4 \mathrm{~A}$ vesicles (and therefore, in the size of the early endosome) was observed, suggesting that Rab 4A might have a direct, active role in the internalization of aSyn.

Recent evidences suggest that even when different RABs localize on the same organelle, they occupy distinct membranes microdomains with different functions, called RAB domains. In particular, some studies have shown that early and recycling endosomes express multiple combinations of Rab 4, Rab 5 and Rab 11 domains that are surely dynamics but still do not significantly intermix over time ${ }^{242}$. Similarly, late endosomes contain distinct membranes domains positive for Rab 7 and Rab 9 respectively (Figure 34).

One of the possible explanation for this segregation mechanism is that it could be, at least in part, mediated by effectors proteins and their association with additional molecules in the organelle membranes ${ }^{242}$.

In the endocytic pathway, early endosome play a fundamental role as a major sorting "hub" from which internalized cargo can be recycled back to the plasma membranes, to the TGN, or routed to lysosome for degradation ${ }^{226}$.

On the early endosomal membranes, Rab5 marks the cisternal domain and Rab4 concentrates in patches that undergo fission from the cisternae, called tubular domains.

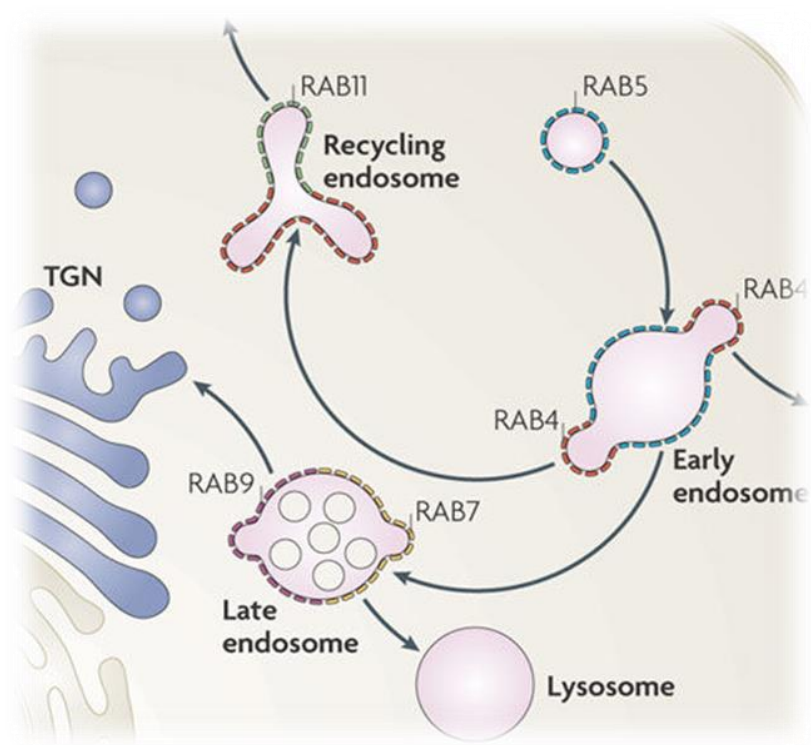

Figure 34. RABs membranes domains.

$\mathrm{RAB}$ microdomains expressed in organelles like recycling endosomes, early endosomes and late endosomes. From Reference 162. 
These tubular subdomains are highly dynamics, giving rise to multiple classes of carrier vesicles that often separate to the endosome and that are targeted to different destinations ${ }^{226}$.

On this behalf, a recent study shown that Rab $4 \mathrm{~A}$ is essential for the recruitment of adaptor proteins to the tubular subdomain of the early endosomal membrane, orchestrating a signalling cascade that initiate the formation of a sorting platform from which multiple classes of vesicular carrier emerge ${ }^{226}$.

In particular, Rab $4 \mathrm{~A}$ is necessary for the recruitment of Arl1, which then promotes assembly of the Arf GEF BIG1 and BIG2, Arfs and adaptor proteins AP1 and AP3 that drive the sorting and packaging of endosomal cargo.

Based on the data obtained, we could clearly observe a perturbation in the morphology of $\mathrm{Rab} 4 \mathrm{~A}$ vesicles due to the presence and the interaction with aSyn monomers.

Thus, we hypothesize that this particular GTPase cascade nucleated by Rab 4A is directly and actively involved into the internalization of aSyn monomers, although further experiments will be necessary to identify the exact molecular link between Rab 4A, Arl1, aSyn and other potential effectors.

\subsection{ASYN IS INTERNALIZED VIA A DYNAMIN-DEPENDENT MECHANISM}

In the last decade, the concept that aSyn has a function in endocytosis solidified, although different views on the exact role played by this protein are emerging.

Studies on triple synuclein knockout mice and on receptor-mediated endocytosis of transferrin suggested a role of aSyn in $\mathrm{CME}^{189,243-245}$, whereas data from other research displayed disturbances in CME and increased bulk endocytosis, proposing instead a compensatory upregulation of CME when aSyn-related fast endocytosis mechanisms are impaired ${ }^{64,246}$.

In this study, we tried to better characterize the mechanism responsible of the internalization of aSyn by using two inhibitors of different endocytic pathways: Dyngo, which is a selective inhibitor of dynamin, and PitStop, which appears to block CME.

The results obtained showed that the inhibition caused by the use of Dyngo has a remarkable effect on the rate of internalized aSyn, both in cultured cells overexpressing 
$\mathrm{Rab} 4 \mathrm{~A}$ - that show an increased internalization of aSyn - and in cultured cells not transfected, suggesting that aSyn is internalized via a dynamin-dependent mechanism. On the other side, the effect observed during the treatment with PitStop were quite controversial. On $\mathrm{H} 4$ cells transfected with Rab 4A, we could observe a small although not significant - decrease in aSyn internalization, while in H4 not transfected cells we noticed an opposite effect, resulting in a bigger uptake of aSyn.

As mentioned before, Dyngo inhibits not only the dynamin-dependent mechanisms, but it was also reported to reduce synaptic vesicle endocytosis and activity-dependent bulk endocytosis.

A possible explanation to the behaviour observed when $\mathrm{H} 4$ cells are treated with PitStop is to be related to a compensatory mechanism: we can hypothesize that when clathrin-mediated mechanisms are blocked (PitStop-related effect), the clathrinindependent processes otherwise suppressed by Dyngo and supposedly, responsible of the internalization of aSyn, - such as synaptic vesicle endocytosis and activitydependent bulk endocytosis - are exacerbated. In this way, a larger amount of aSyn can be internalized. To note, a similar hypothesis was already reported in previous studies $^{246}$.

We can also estimate that the overexpression of Rab 4A would interfere with this equilibrium, and therefore, mitigate the compensatory mechanism aforementioned, so that in the experiments reported in Figure 26 was not possible to observe it so clearly. To conclude, based on the data obtained, we can confirm that a dynamin-dependent mechanism is responsible of the internalization of aSyn monomers, although further experiments will be required to determine in greater details the exact nature of the endocytic process involved.

\subsection{EXtracellular Monomeric aSyn Might Be Sufficient To Initiate The SpREAding OF ASyN PATHOLOGY}

During the last decade, several independent studies demonstrated that aSyn oligomers and pre-formed fibrils (PFFs) enter cultured cells, accumulate into the cytosol or in organelles compartment, and then get released in the extracellular space or transferred from cell-to-cell via several different mechanisms ${ }^{132,134}$. 
Of note, the almost totality of studies present in literature rather focus on the use of oligomeric or already misfolded and aggregating species (such as the widely used PFFs) to study aSyn propagation and its related pathology in vitro and in vivo ${ }^{132,134,141}$. This can be perhaps related to the fact that those same studies report monomeric aSyn is not internalized by cultured cells, or is uptaken much less than aggregates or PFFs species $^{138}$, or that does not propagate as much as the pathogenic ones in vivo.

On the other side, the idea to use and to study the internalization of aSyn monomers came to us from the findings that, whether in a regulated or stochastic way, multiple molecular species of aSyn (and monomers as well) are present in the CSF, in the plasma and in the extracellular space of both healthy and PD individuals ${ }^{144}$.

Thus, we wanted to examine the possibility for regularly available, monomeric aSyn to be uptaken and internalized and to understand whether this could somehow impair the physiological cellular processes.

Our first results confirmed that monomeric aSyn could be indeed internalized in a dose-dependent manner by cultured cells, and that the process occurs via the binding to the plasma membrane. Additionally, the exogenously added aSyn is capable to form high molecular weight species once inside the cells, as indicated by the Dot Blot analysis. The Triton X-100 fractionation confirm and further suggest that those high molecular weight species are, at least in part, insoluble, suggesting that monomeric aSyn might be sufficiently able per se to start to assembly, to eventually misfold, to initiate the process of aggregation and therefore, the spreading of aSyn-related pathology.

Our results do not find, so far, a big correspondence in the literature. Only two studies have reported internalization of aSyn monomers and the involvement of the endocytic pathway, although the purpose and the readouts use in those studies were different ${ }^{140,240}$. In these and in other cases, the discrepancy encountered could be attribute to the different sensitivity of the systems used, to the different models or to the different concentration used and - last but not least - to the focus of the study itself. Nevertheless in $\mathrm{AD}$, a comparable study reported analogous results suggesting that extracellular monomeric tau is sufficient to initiate the spreading of tau pathology ${ }^{247}$. Additionally, we show that aSyn monomers partially colocalize with Rab 4A and Rab $5 \mathrm{~A}$, two small GTPases belonging to the RABs and involved in the endocytic trafficking machinery pathway.

Consistently with the formation of high molecular weight species, it is possible that once in endosomes, aSyn monomers are subjected to a number of physico-chemical transformation that are likely to exert an influence on promoting aggregation reaction. 
In endosomes, $\mathrm{pH}$ is reported to vary between 5.0 and 6.0, while within the lysosomes the variation of $\mathrm{pH}$ is from 4.6 to $5.0 .^{248}$ The low $\mathrm{pH}$ in this compartments might then trigger aggregation, as well as other factors as for example the molecular crowding or the presence of acidic endosomal proteins might contribute to it, suggesting that endocytosis may play a crucial role in the misfolding and aggregation of aSyn, facilitating the propagation of aSyn pathology. It is also possible that co-aggregation occurs in the late endosome-lysosomal compartments, where it appears to end the exogenously added, internalized aSyn monomeric species.

Autophagy function is particularly crucial in the aged brain and in neurodegeneration, due to the accumulation of misfolded and aggregated proteins. Several studies demonstrated that aSyn can be degraded either by the proteasome or by autophagy. In this study, we demonstrate that also high molecular weight species formed via the internalization of aSyn monomeric forms can be degraded by the ALP, and that the concentration of aSyn within the cells is increased when lysosomal activity and protein degradation are blocked. These results support the hypothesis that autophagy is a major route for degradation of aSyn oligomers and fibrillary species when a pathological accumulation is registered ${ }^{146}$.

Based on the results obtained, we proposed a mechanism for aSyn internalization and interaction with the membrane trafficking machinery pathways (Figure 35).

After binding to the membrane, aSyn monomers are internalized through the endocytic pathway, where Rab 4A plays an important role on the protein sorting and on the transport from/to the plasma membrane. aSyn proteins are thereafter found in the early endosome (in colocalization with Rab 5A). It is possible that during the progress from early to late endosome, due to $\mathrm{pH}$ acidification, aSyn monomers start to oligomerize, and therefore to assembly in amyloidogenic aggregates.

From here, the early endosome mature in late endosome and the late endosome where aSyn was found in colocalization with Rab 7 - fuse with lysosome, where aSyn is degraded.

The release of aSyn from those organelles to the cytoplasm, with a consequential accumulation of potential pathological, unfolded and aggregating species it may be possible, facilitating the propagation and the spreading of aSyn pathology. 


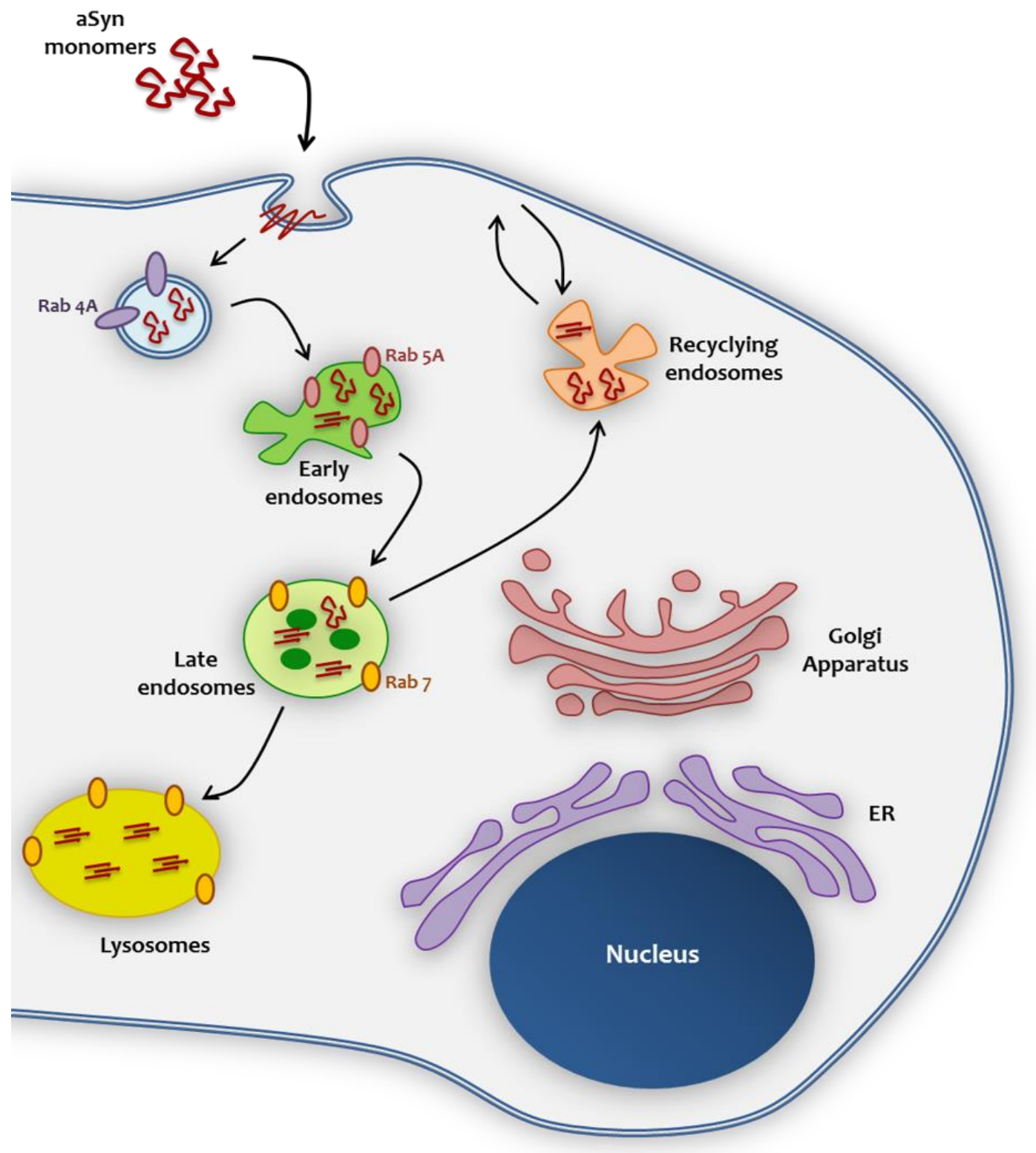

Figure 35. Proposed mechanism for aSyn internalization and interaction with the membrane trafficking machinery pathway. After binding to the membrane, aSyn monomers are internalized through the endocytic pathway. Rab 4A plays an important role on the protein sorting and on the transport from/to the plasma membrane. Monomers are thereafter found in the early endosome. It is possible that during the progress from early to late endosome, due to $\mathrm{pH}$ acidification, aSyn monomers start to oligomerize, and therefore to aggregate in amyloidogenic aggregates. From here, the late endosome fuse with lysosome, where aSyn get degraded. It is possible that in any of those steps a release of aSyn from those organelles to the cytoplasm, with a consequential accumulation of potential pathological, unfolded or oligomerized species will happen. 


\subsection{C-TERminal Modification In SynT And Its EFfect On Aggregation PROPENSITY}

In order to understand the molecular determinants of aSyn aggregation, several in vitro and in vivo models have been employed, in an attempt to mimic the behaviour of the protein in synucleinopathies. However, while aSyn has been known to readily fibrillize in the test tube, it has not been possible to consistently induce aSyn aggregation in cultured cells, just based upon expression.

To promote aSyn aggregation in cells, different strategies have been employed, including co-expression with other proteins ${ }^{249}$, oxidative stress ${ }^{250}$, metal ions ${ }^{251,252}$, or the addition of extraneous amino acid sequences ${ }^{208,253}$. Whether these manipulations mimic relevant aspects of aSyn pathobiology, or simply represent artificial phenotypes that are not accessible to the constellation of conformations aSyn can adopt remains unclear.

In the second part of this study, we characterized and determined the effect of an artificial fragment of EGFP on the C-terminal region of aSyn in terms of its folding and conformation. Our study clarifies the aggregation behaviour of SynT in cells and provides the basis for additional investigations of aSyn aggregation.

From a biophysical perspective, the NMR spectra and general dynamics of the protein backbone observed for aSyn and SynT demonstrate that the aSyn part of SynT retains the intrinsically disordered character of the untagged protein. However, we observed subtle changes in the conformational propensities of the protein ensembles.

As expected, the C-terminal region of aSyn showed changes in the position and intensities of the NMR signals, likely due to changes in the chemical environment induced by the nearby EGFP fragment and its contribution to the local rotational correlation time. On the other hand, we also detected an attenuation of the intensity of the signal of the amide signals in the $\mathrm{N}$-terminal region of SynT that correlated well with a chemical exchange process detected by ${ }^{15} \mathrm{~N}$ spin relaxation measurements.

Taken together, these results suggest that the EGFP fragment might transiently interact with the N-terminus of the protein in an inter- or intra-molecular manner (Figure 36). This transient interaction could explain the attenuated ability of the $\mathrm{N}$-terminal residues of SynT to interact with the small molecule PcTS and could contribute to the altered binding mode of SynT with membranes. We hypothesise that the proximity of the tag could create steric disturbances that influence how SynT interacts with membranes and folds into $\alpha$-helical structures on the membrane surface. 
Considering recent findings that indicate aSyn aggregation is increased when aSyn is in its unstructured monomeric form, and is attenuated/blocked when it is in a alphahelical multimeric form associated with membranes ${ }^{228}$, our results suggest that the perturbed interaction of SynT with membranes might, at least in part, be responsible for its increased aggregation propensity in cells.

It has also been shown that the acidic C-terminal region of aSyn can play a role and affect the aggregation properties of aSyn both in monomeric and aggregated forms ${ }^{36}$. The aggregation kinetics are sensitive to a wide range of ionic conditions, from weak C-terminal charge shielding ${ }^{36}$, to stronger charge modifications caused by the presence of polycations $s^{254}$ or metal ions ${ }^{219,252,251,224}, \mathrm{PTMs}^{224,255}$, truncations ${ }^{256,257}$ or, as in the case of our study, the addition of tags ${ }^{208}$. Thus, it is possible that changes in the electrostatic environment of this region of the protein due to the presence of the tag will overall affect the aggregation behaviour.

The present study indicates that the modification of the C-terminus of aSyn in SynT, and the subsequent effect on the $\mathrm{N}$-terminal region, potentiates the ability of the protein to form intracellular inclusions, which recapitulate important molecular features of synucleinopathies.

Cellular models based on expression of aSyn variants such as SynT are widely used tools for the identification of genetic and pharmacological strategies to treat PD and other synucleinopathies. Therefore, a detailed understanding of the molecular mechanisms underlying aSyn aggregation is important for the development of novel therapeutic strategies for these disorders. 

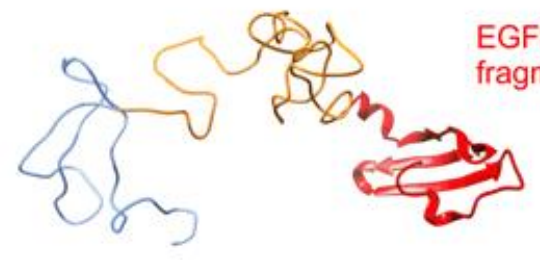

aSyn
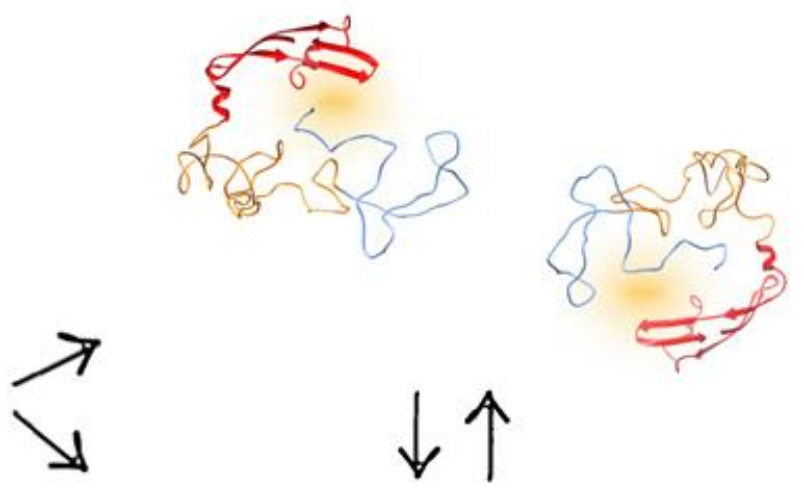

INTERMOLECULAR INTERACTIONS

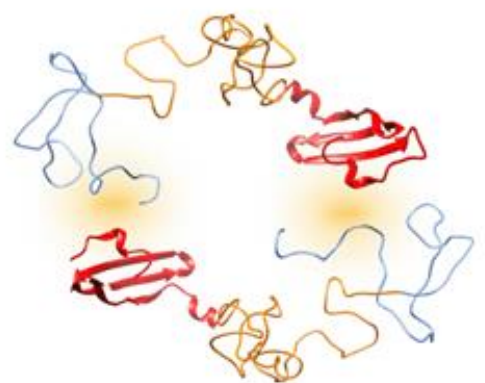

Figure 36. Proposed model for the structural properties of SynT.

The EGFP fragment could transiently interact with the $\mathrm{N}$-terminal region of the protein in an inter-molecular or in an intra-molecular way. Overall, the general, chemical properties of aSyn are not affected by the tag, nor its propensity to maintain an intrinsically disordered structure. Still, some of the physiological-related properties of aSyn appear to be affected by the tag (e.g., membrane binding properties and aggregation propensities in vivo and in vitro). 
Discussion 


\section{- VI - \\ CONCLUSIONS}




\section{CONCLUSIONS}

In this study, we analysed the mechanisms of aSyn interaction with the plasma membrane and with cellular trafficking machinery components during the internalization of exogenously-added aSyn recombinant proteins in a cell model. We also characterized the structural basis behind the aggregation process in a $\mathrm{C}$-terminally modified version of aSyn, SynT, which mimic the pathological behaviour of the protein.

First, we demonstrated that different species of exogenously added aSyn, in the form of monomers and fibrils, enter cultured cells and form inclusions.

Once uptaken, aSyn monomers display a distinct, perinuclear and punctuate pattern, suggesting that these may localize in vesicular structures.

Throughout the screening of a family of small GTPases, the RABs, we could demonstrate that aSyn partially colocalize with $\mathrm{Rab} 5 \mathrm{~A}$ and Rab 7, suggesting the involvement of the endocytic pathway first, and of the ALP later during the processing of aSyn.

Internalization of aSyn is remarkably increased by the overexpression of Rab $4 \mathrm{~A}$, which orchestrates a signalling cascade for the recruitment of adaptor proteins to the early endosomes. This let us hypothesize that this particular GTPase cascade nucleated by $\mathrm{Rab} 4 \mathrm{~A}$ is directly and actively involved into the internalization of aSyn monomers, although further experiments will be necessary to identify the exact molecular link between Rab 4A, aSyn monomers and other potential effectors.

Additional investigations with different mutants of aSyn demonstrated that membrane binding properties are essential for the internalization, and therefore, for the inclusion formation of aSyn in cultured cells.

By using selective inhibitors of the endocytic and lysosomal pathways, we demonstrated that aSyn monomers are internalized via a dynamin-dependent process, but not throughout CME. We also could prove the involvement of the ALP as a selected mechanism in the intracellular degradation of aSyn.

Based on the results obtained, we claim that extracellular monomeric aSyn might be sufficient to initiate the spreading of aSyn pathology, and we proposed a detailed mechanism for aSyn internalization and processing in cultured cells.

In the second part of the study, we characterize the biophysical properties of an aggregation-prone form of aSyn, known as SynT. 
We performed a detailed structural characterization of SynT through a systematic comparison with the unmodified aSyn and with the use of NMR-based techniques.

Our data suggests that the intrinsically disordered nature of aSyn is not strongly influenced by the C-terminal modification in SynT, and that the conformations adopted by SynT are similar to those described for the unmodified protein. Nevertheless, a few differences were observed at the N-terminal region, and the transient interactions between the $\mathrm{N}$-terminal and the C-terminal regions in SynT resulted strengthened if compared to aSyn.

The use of small molecules as a structural probe (such as PcTS) as well as the membrane-binding assay performed helped us to further demonstrate that the $\mathrm{N}$ terminal region of SynT is less available than in aSyn, suggesting that disturbances in the $\mathrm{N}$-terminal region and a consequent decrease of membrane-binding properties might contribute to the pathobiology of aSyn.

Our data provide insight into the effect of the EGFP fragment on the N-terminus of the protein, in an inter- or intra-molecular fashion. The mechanism proposed indicates how the modification of the C-terminus of aSyn in SynT, and the subsequent effect on the N-terminal region potentiates the ability of the protein to form intracellular inclusions, which recapitulate important molecular features of synucleinopathies. 


$$
\text { - VII - }
$$

BIBLIOGRAPHY 


\section{BIBLIOGRAPHY}

1. Kontis, V. et al. Future life expectancy in 35 industrialised countries: projections with a Bayesian model ensemble. Lancet 389, 1323-1335 (2017).

2. Vaupel, J. W. Biodemography of human ageing. Nature 464, 536-542 (2010).

3. Christensen, K., Doblhammer, G., Rau, R. \& Vaupel, J. W. Ageing populations: the challenges ahead. Lancet 374, 1196-1208 (2009).

4. Driver, J. A., Djousse, L., Logroscino, G., Gaziano, J. M. \& Kurth, T. Incidence of cardiovascular disease and cancer in advanced age: prospective cohort study. Bmj 337, a2467-a2467 (2008).

5. Jin, K., Simpkins, J. W., Ji, X., Leis, M. \& Stambler, I. The Critical Need to Promote Research of Aging and Aging-related Diseases to Improve Health and Longevity of the Elderly Population. Aging Dis. 6, 1 (2015).

6. $\quad$ Lee, A. \& Gilbert, R. M. Epidemiology of Parkinson Disease. Neurol. Clin. 34, 955965 (2016).

7. Mhyre, T. R., Nw, R., Boyd, J. T., Hall, G. \& Room, C. Parkinson's Disease. 65, (2012).

8. Gallagher, D. A., Lees, A. J. \& Schrag, A. What are the most important nonmotor symptoms in patients with Parkinson's disease and are we missing them? Mov. Disord. 25, 2493-2500 (2010).

9. Simuni, T. \& Sethi, K. Nonmotor manifestations of Parkinson's disease. Ann. Neurol. 64, 65-80 (2008).

10. Wales, P., Lázaro, D. F., Pinho, R. \& Outeiro, T. F. Limelight on alpha-synuclein: Pathological and mechanistic implications in neurodegeneration. J. Parkinsons. Dis. 3, 415-459 (2013).

11. Braak, H. et al. Staging of brain pathology related to sporadic Parkinson's disease. Neurobiol. Aging 24, 197-211 (2003).

12. Del Tredici, K. \& Braak, H. Sporadic Parkinson's disease: Development and distribution of $\alpha$-synuclein pathology. Neuropathol. Appl. Neurobiol. 42, 33-50 (2016).

13. Goedert, M., Spillantini, M. G., Del Tredici, K. \& Braak, H. 100 years of Lewy pathology. Nat. Rev. Neurol. 9, 13-24 (2012).

14. Polymeropoulos, M. H. Mutation in the -Synuclein Gene Identified in Families with Parkinson's Disease. Science (80-. ). 276, 2045-2047 (1997).

15. Golde, T. E., Borchelt, D. R., Giasson, B. I. \& Lewis, J. Thinking laterally about neurodegenerative proteinopathies. J. Clin. Invest. 123, 1847-1855 (2013).

16. Bayer, T. A. Proteinopathies, a core concept for understanding and ultimately treating degenerative disorders? Eur. Neuropsychopharmacol. 25, 713-724 (2015).

17. Goedert, M., Spillantini, M. G. \& Davies, S. W. Filamentous nerve cell inclusions in 
neurodegenerative diseases. Curr. Opin. Neurobiol. 8, 619-632 (1998).

18. Spillantini, M. G., Goedert, M. \& Spillantini, M. G. The $\alpha$-Synucleinopathies: Parkinson's Disease, Dementia with Lewy Bodies, and Multiple System Atrophy. Ann. N. Y. Acad. Sci. 920, 16-27 (2000).

19. John Q. Trojanowski, V. M.-Y. L. Parkinson's disease and related alphasynucleiopathies are brain amyloidoses. Ann N.Y. Acad. Sci 991, 107-110 (2003).

20. Spillantini, M. G., Schmidt, M. L., Lee, V. M.-Y., Trojanowski, J. Q. \& Goedert, M. a-Synuclein in Lewy bodies. Nature 839-840 (1997).

21. Wakabayashi, K., Yoshimoto, M., Tsuji, S. \& Takahashi, H. a -Synuclein immunoreactivity in glial cytoplasmic inclusions in multiple system atrophy. Neurosci. Lett. 249, 180-182 (1998).

22. Trojanowski, J. Q., Goedert, M., Iwatsubo, T. \& Lee, V. M. Fatal attractions: abnormal protein aggregation and neuron death in Parkinson's disease and Lewy body dementia. Cell Death Differ. 5, 832-837 (1998).

23. Spillantini, M. G., Crowther, R. A., Jakes, R., Hasegawa, M. \& Goedert, M. alphaSynuclein in filamentous inclusions of Lewy bodies from Parkinson's disease and dementia with lewy bodies. Proc. Natl. Acad. Sci. U. S. A. 95, 6469-73 (1998).

24. Takeda, A. et al. Abnormal accumulation of NACP/ $\alpha$-synuclein in neurodegenerative disorders. Am. J. Pathol. 152, 367-372 (1998).

25. Maroteaux, Luc., J. T. C. and R. H. S. Synuclein : A Neuron-Specific Protein Localized to the Nucleus and Presynaptic Nerve Terminal. J. Neurosci. 8, 2804-2815 (1988).

26. Chandra, S., Chen, X., Rizo, J., Jahn, R. \& Su, T. C. A Broken a-Helix in Folded aSynuclein. J. Biol. Chem. 278, 15313-15318 (2003).

27. Bussell, R. J. \& Eliezer, D. A Structural and Functional Role for 11-mer Repeats in a Synuclein and Other Exchangeable Lipid Binding Proteins. J. Mol. Biol. 2836, 763778 (2003).

28. Davidson, W. S., Jonas, a, Clayton, D. F. \& George, J. M. Stabilization of alphasynuclein secondary structure upon binding to synthetic membranes. J. Biol. Chem. 273, 9443-9 (1998).

29. Giasson, B. I., Murray, I. V. J., Trojanowski, J. Q. \& Lee, V. M. A Hydrophobic Stretch of 12 Amino Acid Residues in the Middle of a-Synuclein Is Essential for Filament Assembly. J. Biol. Chem. 276, 2380-2386 (2001).

30. Du, H. et al. A Peptide Motif Consisting of Glycine, Alanine, and Valine Is Required for the fibrillization and Cytotoxicity of Human a-Synuclein. Biochemistry 8870-8878 (2003).

31. Ueda, K. et al. Molecular cloning of cDNA encoding an unrecognized component of amyloid in Alzheimer disease. Proc. Natl. Acad. Sci. 90, 11282-11286 (1993).

32. Bayer, T. A. et al. $\alpha$-Synuclein accumulates in Lewy bodies in Parkinson's disease and dementia with Lewy bodies but not in Alzheimer's disease $\beta$-amyloid plaque cores. Neurosci. Lett. 266, 213-216 (1999). 
33. Souza, M. Y., Giasson, B. I., Lee, V. M. \& Y, H. I. Chaperone-like activity of synucleins. FEBS Lett. 474, 116-119 (2000).

34. Kim, T. D., Paik, S. R. \& Yang, C.-H. Structural and functional implications of Cterminal regions of alpha-synuclein. Biochemistry 41, 13782-13790 (2002).

35. Park, S. M. et al. Distinct Roles of the N-terminal-binding Domain and the Cterminal-solubilizing Domain of A-Synuclein, a Molecular Chaperone. J. Biol. Chem. 277, 28512-28520 (2002).

36. Hoyer, W., Cherny, D., Subramaniam, V. \& Jovin, T. M. Impact of the Acidic CTerminal Region Comprising Amino Acids 109 - 140 on a-Synuclein Aggregation in Vitro. Biochemistry 16233-16242 (2004).

37. Eliezer, D., Kutluay, E., Bussell, R. \& Browne, G. Conformational properties of $\alpha$ synuclein in its free and lipid-associated states. J. Mol. Biol. 307, 1061-1073 (2001).

38. Breydo, L., Wu, J. W. \& Uversky, V. N. $\alpha$-Synuclein misfolding and Parkinson's disease. Biochim. Biophys. Acta - Mol. Basis Dis. 1822, 261-285 (2012).

39. Cho, M. K. et al. Amino acid bulkiness defines the local conformations and dynamics of natively unfolded $\alpha$-synuclein and tau. J. Am. Chem. Soc. 129, 3032-3033 (2007).

40. Allison, J. R., Varnai, P., Dobson, C. M. \& Vendruscolo, M. Determination of the free energy landscape of alpha-synuclein using spin label nuclear magnetic resonance measurements. J. Am. Chem. Soc. 131, 18314-18326 (2009).

41. Salmon, L. et al. NMR characterization of long-range order in intrinsically disordered proteins. J Am Chem Soc 132, 8407-8418 (2010).

42. Rao, J. N., Jao, C. C., Hegde, B. G., Langen, R. \& Ulmer, T. S. A combinatorial NMR and EPR approach for evaluating the structural ensemble of partially folded proteins. J. Am. Chem. Soc. 132, 8657-8668 (2010).

43. Lee, J. C., Bert, L. T., Kozak, J. J., Gray, H. B. \& Winkler, J. R. a-Synuclein tertiary contact dynamics. $J$ phys Chem B 2017-2112 (2007). doi:10.1038/nn.2120.Red-shifted

44. Dedmon, M. M., Lindorff-Larsen, K., Christodoulou, J., Vendruscolo, M. \& Dobson, C. M. Mapping long-range interactions in alpha-synuclein using spin-label NMR and ensemble molecular dynamics simulations. J. Am. Chem. Soc. 127, 476-477 (2005).

45. Bertoncini, C. W. et al. From The Cover: Release of long-range tertiary interactions potentiates aggregation of natively unstructured -synuclein. Proc. Natl. Acad. Sci. 102, 1430-1435 (2005).

46. Uversky, V. N. Neuropathology, biochemistry, and biophysics of a-synuclein aggregation. J. Neurochem. 103, 17-37 (2007).

47. Bussell, R. \& Eliezer, D. Residual Structure and Dynamics in Parkinson's Diseaseassociated Mutants of a-Synuclein. J. Biol. Chem. 276, 45996-46003 (2001).

48. Ulmer, T. S. \& Bax, A. Comparison of structure and dynamics of micelle-bound human $\alpha$-synuclein and Parkinson disease variants. J. Biol. Chem. 280, 43179-43187 (2005).

49. Iwai, A. et al. The precursor protein of non-AB component of Alzheimer's disease amyloid is a presynaptic protein of the central nervous system. Neuron 14, 467-475 
(1995).

50. Tyson, T., Steiner, J. A. \& Brundin, P. Sorting out release, uptake and processing of alpha-synuclein during prion-like spread of pathology. J. Neurochem. 139, 275-289 (2016).

51. Bartels, T., Choi, J. G., Selkoe, D. J. \& Hospital, W. That Resists Aggregation. 477, 107-110 (2012).

52. Wang, W. et al. A soluble a-synuclein construct forms a dynamic tetramer. Proc. Natl. Acad. Sci. 108, 17797-17802 (2011).

53. Burré, J., Sharma, M. \& Südhof, T. C. a-Synuclein assembles into higher-order multimers upon membrane binding to promote SNARE complex formation. Proc. Natl. Acad. Sci. (2014). doi:10.1073/pnas.1416598111

54. Payton, J. E., Perrin, R. J., Woods, W. S. \& George, J. M. Structural determinants of PLD2 inhibition by a-synuclein. J. Mol. Biol. 337, 1001-1009 (2004).

55. Withers, G. S., George, J. M., Banker, G. A. \& Clayton, D. F. Delayed localization of synelfin (synuclein, NACP) to presynaptic terminals in cultured rat hippocampal neurons. Dev. Brain Res. 99, 87-94 (1997).

56. Kahle, P. J. et al. Subcellular localization of wild-type and Parkinson's diseaseassociated mutant alpha -synuclein in human and transgenic mouse brain. J. Neurosci. 20, 6365-6373 (2000).

57. Lee, S.-J., Jeon, H. \& Kandror, K. V. Alpha-synuclein is localized in a subpopulation of rat brain synaptic vesicles. Acta Neurobiol. Exp. (Wars). 68, 509-15 (2008).

58. Scott, D. A., Tabarean, I., Tang, Y., Cartier, A. \& Masliah, E. a pathologic cascade leading to synaptic dysfunction in a-synuclein induced neurodegeneration. J. Neurosci. 30, 8083-8095 (2010).

59. Abeliovich, A. et al. Mice Lacking $\alpha$-Synuclein Display Functional Deficits in the Nigrostriatal Dopamine System. Neuron 25, 239-252 (2000).

60. Nemani, V. M. et al. NIH Public AccessIncreased Expression of Alpha-Synuclein Reduces Neurotransmitter Release by Inhibiting Synaptic Vesicle Reclustering After Endocytosis. Neuron 65, 66-79 (2011).

61. Gaugler, M. N. et al. Nigrostriatal overabundance of $\alpha$-synuclein leads to decreased vesicle density and deficits in dopamine release that correlate with reduced motor activity. Acta Neuropathol. 123, 653-669 (2012).

62. Lundblad, M., Decressac, M., Mattsson, B. \& Bjorklund, A. Impaired neurotransmission caused by overexpression of -synuclein in nigral dopamine neurons. Proc. Natl. Acad. Sci. 109, 3213-3219 (2012).

63. Burré, J., Sharma, M., Tsetsenis, T., Buchman, V. \& Südhof, T. C. $\alpha$-Synuclein Promotes SNARE-Complex Assembly in vivo and in vitro. Science (80-. ). 329, 16631667 (2010).

64. Lautenschläger, J., Kaminski, C. F. \& Kaminski Schierle, G. S. a-Synuclein Regulator of Exocytosis, Endocytosis, or Both? Trends Cell Biol. 27, (2017).

65. Lai, Y. et al. Nonaggregated $\alpha$-synuclein influences snare-dependent vesicle docking 
via membrane binding. Biochemistry 53, 3889-3896 (2014).

66. Choi, B.-K. et al. Large -synuclein oligomers inhibit neuronal SNARE-mediated vesicle docking. Proc. Natl. Acad. Sci. 110, 4087-4092 (2013).

67. DeWitt, D. C. \& Rhoades, E. a-synuclein can inhibit SNARE-mediated Vesicle Fusion through direct interaction with lipid bilayers. Biochemistry 119, 5124-5136 (2010).

68. Kamp, F. \& Beyer, K. Binding of a-synuclein affects the lipid packing in bilayers of small vesicles. J. Biol. Chem. 281, 9251-9259 (2006).

69. Fusco, G. et al. Structural Ensembles of Membrane-bound $\alpha$-Synuclein Reveal the Molecular Determinants of Synaptic Vesicle Affinity. Sci. Rep. 6, 27125 (2016).

70. Uversky, V. N., Li, J. \& Fink, A. L. Metal-triggered structural transformations, aggregation, and fibrillation of human $\alpha$-synuclein: A possible molecular link between parkinson's disease and heavy metal exposure. J. Biol. Chem. 276, 44284-44296 (2001).

71. Binolfi, A. et al. Interaction of a-Synuclein with Divalent Metal Ions Reveals Key Differences : A Link between Structure, Binding Specificity and Fibrillation Enhancement. J. Am. Chem. Soc. 2045-2047 (2006).

72. Snead, D. \& Eliezer, D. Alpha-Synuclein Function and Dysfunction on Cellular Membranes. Exp. Neurobiol. 23, 292 (2014).

73. Ly, T. \& Julian, R. R. Protein-Metal Interactions of Calmodulin and a-Synuclein Monitored by Selective Noncovalent Adduct Protein Probing Mass Spectrometry. $J$. Am. Soc. Mass Spectrom. 19, 1663-1672 (2008).

74. Lee, D., Lee, S. Y., Lee, E. N., Chang, C. S. \& Paik, S. R. a-Synuclein Exhibits Competitive Interaction Between Calmodulin and Synthetic Membranes. $J$. Neurochem. 82, 1007-1017 (2002).

75. Martinez, J., Moeller, I., Erdjument-Bromage, H., Tempst, P. \& Lauring, B. Parkinson's disease-associated $\alpha$-synuclein is a calmodulin substrate. J. Biol. Chem. 278, 17379-17387 (2003).

76. Wersinger, C. \& Sidhu, A. Attenuation of dopamine transporter activity by $\alpha$ synuclein. Neurosci. Lett. 340, 189-192 (2003).

77. Krüger, R. et al. Ala30Pro mutation in the gene encoding a-synuclein in Parkinson's. Nat. Genet. 18, 231-236 (1998).

78. Zarranz, J. J. et al. The new mutation, E46K, of alpha-synuclein causes Parkinson and Lewy body dementia. Ann. Neurol. 55, 164-73 (2004).

79. Appel-Cresswell, S. et al. Alpha-synuclein p.H50Q, a novel pathogenic mutation for Parkinson's disease. Mov. Disord. 28, 811-813 (2013).

80. Lesage, S. et al. G51D $\alpha$-synuclein mutation causes a novel Parkinsonian-pyramidal syndrome. Ann. Neurol. 73, 459-471 (2013).

81. Pasanen, P. et al. A novel $\alpha$-synuclein mutation A53E associated with atypical multiple system atrophy and Parkinson's disease-type pathology. Neurobiol. Aging 35, $1-5$ (2014). 
82. Chartier-Harlin, M.-C. et al. $\alpha$-synuclein locus duplication as a cause of familial Parkinson's disease. Lancet 364, 1169-1171 (2004).

83. Singleton, a B. et al. a-Synuclein Locus Triplication Causes Parkinson' s Disease. Science (80-. ). 302, 841 (2003).

84. Rutherford, N. J., Moore, B. D., Golde, T. E. \& Giasson, B. I. Divergent effects of the H50Q and G51D SNCA mutations on the aggregation of $\alpha$-synuclein. $J$. Neurochem. 131, 859-867 (2015).

85. Ono, K., Ikeda, T., Takasaki, J. ichi \& Yamada, M. Familial Parkinson disease mutations influence ??-Synuclein assembly. Neurobiol. Dis. 43, 715-724 (2011).

86. Narhi, L. et al. Both familial Parkinson's disease mutations accelerate $\alpha$-synuclein aggregation. J. Biol. Chem. 274, 9843-9846 (1999).

87. Conway, K. A. et al. Acceleration of oligomerization, not fibrillization, is a shared property of both alpha -synuclein mutations linked to early-onset Parkinson's disease: Implications for pathogenesis and therapy. Proc. Natl. Acad. Sci. 97, 571-576 (2000).

88. Bussel, R. \& Eliezer, D. Effects of Parkinson' s Disease-Linked Mutations on the Structure of lipid-associated a-Synuclein. Biochemistry 4810-4818 (2004).

89. Jo, E., Fuller, N., Rand, R. P., St George-Hyslop, P. \& Fraser, P. E. Defective membrane interactions of familial Parkinson's disease mutant A30P $\alpha$-synuclein 1 1Edited by I. B. Holland. J. Mol. Biol. 315, 799-807 (2002).

90. Jensen, P. H., Nielsen, M. S., Jakes, R., Dotti, G. \& Goedert, M. Binding of alphasynuclein to brain vesicles is abolished by familial parkinsons-disease mutation. J. Biol. Chem. 273, 26292-26294 (1998).

91. Bodner, C. R., Maltsev, A. S., Dobson, C. M. \& Bax, A. Differential phospholipid binding of $\alpha$-synuclein variants implicated in Parkinson's disease revealed by solution NMR spectroscopy. Biochemistry 49, 862-871 (2010).

92. Choi, W. et al. Mutation E46K increases phospholipid binding and assembly into filaments of human $\alpha$-synuclein. FEBS Lett. 576, 363-368 (2004).

93. Khalaf, O. et al. The H50Q mutation enhances $\alpha \alpha$-synuclein aggregation, secretion, and toxicity. J. Biol. Chem. 289, 21856-21876 (2014).

94. Oueslati, A. Implication of Alpha-Synuclein Phosphorylation at S129 in Synucleinopathies: What Have We Learned in the Last Decade? J. Parkinsons. Dis. 6, 39-51 (2016).

95. Nonaka, T., Iwatsubo, T. \& Hasegawa, M. Ubiquitination of alpha-synuclein. Biochemistry 44, 361-368 (2005).

96. Krumova, P. et al. Sumoylation inhibits $\alpha$-synuclein aggregation and toxicity. J. Cell Biol. 194, 49-60 (2011).

97. Vicente Miranda, H. et al. Glycation potentiates $\alpha$-synuclein-associated neurodegeneration in synucleinopathies. Brain 140, 1399-1419 (2017).

98. de Oliveira, R. M. et al. The mechanism of sirtuin 2-mediated exacerbation of alphasynuclein toxicity in models of Parkinson disease. PLoS Biol. 15, 1-27 (2017). 
99. Glaser, C. B., Yamin, G., Uversky, V. N. \& Fink, A. L. Methionine oxidation, $\alpha$ synuclein and Parkinson's disease. Biochim. Biophys. Acta - Proteins Proteomics 1703, 157-169 (2005).

100. Beyer, K. $\alpha$-Synuclein structure, posttranslational modification and alternative splicing as aggregation enhancers. Acta Neuropathol. 112, 237-251 (2006).

101. Chen, L. \& Feany, M. B. $\alpha$-Synuclein phosphorylation controls neurotoxicity and inclusion formation in a Drosophila model of Parkinson disease. Nat. Neurosci. 8, 657663 (2005).

102. Fujiwara, H. et al. $\alpha$-Synuclein is phosphorylated in synucleinopathy lesions. Nat. Cell Biol. 4, 160-164 (2002).

103. Nishie, M. et al. Accumulation of phosphorylated a-synuclein in the brain and peripheral ganglia of patients with multiple system atrophy. Acta Neuropathol. 107, 292-298 (2004).

104. da Fonseca, T. L., Villar-Piqué, A. \& Outeiro, T. F. The interplay between alphasynuclein clearance and spreading. Biomolecules 5, 435-471 (2015).

105. Fauvet, B. et al. Characterization of Semisynthetic and Naturally N-Acetylated aSynuclein in Vitro and in Intact Cells. J. Biol. Chem. 287, 28243-28262 (2012).

106. Maltsev, A. S., Ying, J. \& Bax, A. Impact of N-terminal acetylation of a-synuclein on its random coil and lipid binding properties. Biochemistry 51, 5004-5013 (2012).

107. Kang, L. et al. N-terminal acetylation of a-synuclein induces increased transient helical propensity and decreased aggregation rates in the intrinsically disordered monomer. Protein Sci. 21, 911-917 (2012).

108. Uversky, V. N., Li, J. \& Fink, A. L. Evidence for a Partially Folded Intermediate in ??-Synuclein Fibril Formation. J. Biol. Chem. 276, 10737-10744 (2001).

109. Souza, J. M., Giasson, B. I., Chen, Q., Lee, V. M. Y. \& Ischiropoulos, H. Dityrosine cross-linking promotes formation of stable $\alpha$-synuclein polymers: Implication of nitrative and oxidative stress in the pathogenesis of neurodegenerative synucleinopathies. J. Biol. Chem. 275, 18344-18349 (2000).

110. Ding, T. T., Lee, S., Rochet, J. \& Lansbury, P. T. Annular a-Synuclein Protofibrils Are Produced When Spherical Protofibrils Are Incubated in Solution or Bound to Brain-Derived Membranes †. Biochemistry 10209-10217 (2002).

111. Volles, M. J. et al. Vesicle permeabilization by protofibrillar alpha-synuclein: implications for the pathogenesis and treatment of Parkinson's disease. Biochemistry 40, 7812-7819 (2001).

112. Volles, M. J. \& Lansbury, P. T. Vesicle permeabilization by protofibrillar $\alpha$-synuclein is sensitive to Parkinson's disease-linked mutations and occurs by a pore-like mechanism. Biochemistry 41, 4595-4602 (2002).

113. Wood, S. J. et al. a-Synuclein Fibrillogenesis is Nucleation-dependent. Biochemistry 19509-19512 (1999). doi:10.1074/jbc.274.28.19509

114. Gosavi, N., Lee, H. J., Lee, J. S., Patel, S. \& Lee, S. J. Golgi fragmentation occurs in the cells with prefibrillar??-synuclein aggregates and precedes the formation of fibrillar inclusion. J. Biol. Chem. 277, 48984-48992 (2002). 
115. $\mathrm{Xu}$, J. et al. Dopamine-dependent neurotoxicity of alpha-synuclein: a mechanism for selective neurodegeneration in Parkinson disease. Nat. Med. 8, 600-606 (2002).

116. Danzer, K. M. et al. Different species of $\alpha$-synuclein oligomers induce calcium influx and seeding. J. Neurosci. 27, 9220-9232 (2007).

117. Hsu, L. J. et al. A-Synuclein Promotes Mitochondrial Deficit and Oxidative Stress. Am. J. Pathol. 157, 401-410 (2000).

118. Hashimoto, M. et al. The Role of alpha-synuclein assembly and metabolism in the pathogenesis of Lewy body disease. J. Mol. Neurosci. 24, 343-52 (2004).

119. Alim, M. A. et al. Demonstration of a role for a-synuclein as a functional microtubuleassociated protein. J. Alzheimer's Dis. 6, 435-442 (2004).

120. Takeda, S. et al. Neuronal uptake and propagation of a rare phosphorylated highmolecular-weight tau derived from Alzheimer's disease brain. Nat. Commun. 6, 8490 (2015).

121. Lashuel, H. A., Overk, C. R., Oueslati, A. \& Masliah, E. The many faces of $\alpha-$ synuclein: from structure and toxicity to therapeutic target. Nat. Rev. Neurosci. 14, 3848 (2013).

122. Braak, H., Ghebremedhin, E., Rüb, U., Bratzke, H. \& Del Tredici, K. Stages in the development of Parkinson's disease-related pathology. Cell Tissue Res. 318, 121-134 (2004).

123. Hawkes, C. H., Del Tredici, K. \& Braak, H. Parkinson's disease: A dual-hit hypothesis. Neuropathol. Appl. Neurobiol. 33, 599-614 (2007).

124. Kordower, J. H., Chu, Y., Hauser, R. A., Freeman, T. B. \& Olanow, C. W. Lewy body-like pathology in long-term embryonic nigral transplants in Parkinson's disease. Nat. Med. 14, 504-506 (2008).

125. Li, J. Y. et al. Lewy bodies in grafted neurons in subjects with Parkinson's disease suggest host-to-graft disease propagation. Nat. Med. 14, 501-503 (2008).

126. Brundin, P., Li, J., Holton, J. L., Lindvall, O. \& Revesz, T. Research in motion: the enigma of Parkinson's disease pathology spread. Nat. Rev. Neurosci. 9, 741-745 (2008).

127. Brundin, P., Melki, R. \& Kopito, R. Prion-like transmission of protein aggregates in neurodegenerative disorders. Nat. Rev. Mol. Cell Biol. 11, 301-307 (2010).

128. Brundin, P. \& Melki, R. Prying into the Prion Hypothesis for Parkinson's Disease. $J$. Neurosci. 37, 9808-9818 (2017).

129. Lee, H. J. et al. Assembly-dependent endocytosis and clearance of extracellular $\alpha$ synuclein. Int. J. Biochem. Cell Biol. 40, 1835-1849 (2008).

130. Danzer, K. M., Krebs, S. K., Wolff, M., Birk, G. \& Hengerer, B. Seeding induced by a-synuclein oligomers provides evidence for spreading of a-synuclein pathology. $J$. Neurochem. 111, 192-203 (2009).

131. Desplats, P. et al. Inclusion Formation and Neuronal Cell Death through Neuron-toNeuron Transmission of Alpha-Synuclein. J. Neurochem. 110, 137 (2009).

132. Luk, K. C. et al. Exogenous alpha-synuclein fibrils seed the formation of Lewy body- 
like intracellular inclusions in cultured cells. Proc. Natl. Acad. Sci. U. S. A. 106, 20051-6 (2009).

133. Hansen, C. et al. $\alpha$-Synuclein propagates from mouse brain to grafted dopaminergic neurons and seeds aggregation in cultured human cells. 121, 715-725 (2011).

134. Volpicelli-daley, L. a et al. exogenous aSynuclein fibrils induce Lewy Body Pathology leading to Synaptic Dysfunction and Neuron Death. Neuron 72, 57-71 (2011).

135. Freund, E. C. et al. neuron-to-neuron transmission of aSynuclein fibrils through axonal transport. 72, 517-524 (2013).

136. Reyes, J. F. et al. A cell culture model for monitoring $\alpha$-synuclein cell-to-cell transfer. Neurobiol. Dis. 77, 266-275 (2015).

137. Shrivastava, A. N. et al. aSynuclein assemblies sequester neuronal a3-Na+/K + ATPase and impair $\mathrm{Na}+$ gradient. EMBO J. 34, 2408-2423 (2015).

138. Mao, X. et al. Pathological a-synuclein transmission initiated by binding lymphocyteactivation gene 3. Science (80-. ). 353, aah3374-aah3374 (2016).

139. Holmes, B. B. et al. Heparan sulfate proteoglycans mediate internalization and propagation of specific proteopathic seeds. Proc. Natl. Acad. Sci. 110, E3138-E3147 (2013).

140. Sung, J. Y. et al. Induction of Neuronal Cell Death by Rab5A-dependent Endocytosis of a-Synuclein. J. Biol. Chem. 276, 27441-27448 (2001).

141. Luk, K. C. et al. pathological a-synuclein transmission initiates parkinson-like Neurodegeneration in non-transgenic mice. Science (80-. ). 338, 949-953 (2013).

142. Guo, J. L. \& Lee, V. M. Y. Cell-to-cell transmission of pathogenic proteins in neurodegenerative diseases. Nat. Med. 20, 130-138 (2014).

143. Abounit, S. et al. Tunneling nanotubes spread fibrillar $\alpha$-synuclein by intercellular trafficking of lysosomes. EMBO J. 35, 2120-2138 (2016).

144. El-Agnaf, O. M. A. -Synuclein implicated in Parkinson's disease is present in extracellular biological fluids, including human plasma. FASEB J. 1945-1947 (2003). doi:10.1096/fj.03-0098fje

145. Emmanouilidou, E. et al. Cell-Produced -Synuclein Is Secreted in a CalciumDependent Manner by Exosomes and Impacts Neuronal Survival. J. Neurosci. 30, 6838-6851 (2010).

146. Danzer, K. M. et al. Exosomal cell-to-cell transmission of alpha synuclein oligomers. Mol. Neurodegener. 7, 42 (2012).

147. Sacino, A. N. et al. Amyloidogenic a-synuclein seeds do not invariably induce rapid, widespread pathology in mice. Acta Neuropathol. 127, 645-665 (2014).

148. Recasens, A. et al. Lewy body extracts from Parkinson disease brains trigger $\alpha$ synuclein pathology and neurodegeneration in mice and monkeys. Ann. Neurol. 75, 351-362 (2014).

149. Peelaerts, W. et al. a-Synuclein strains cause distinct synucleinopathies after local and systemic administration. Nature 522, 340-344 (2015). 
150. Dunning, C. J. R., George, S. \& Brundin, P. What's to like about the prion-like hypothesis for the spreading of aggregated $\alpha$-synuclein in Parkinson disease? Prion 7 , 92-7 (2013).

151. Melki, R. Role of different alpha-synuclein strains in synucleinopathies, similarities with other neurodegenerative diseases. J. Parkinsons. Dis. 5, 217-227 (2015).

152. Ferreon, A. C. M., Gambin, Y., Lemke, E. A. \& Deniz, A. A. Interplay of asynuclein binding and conformational switching probed by single-molecule fluorescence. Proc. Natl. Acad. Sci. 106, 5645-5650 (2009).

153. Jao, C. C., Hegde, B. G., Chen, J., Haworth, I. S. \& Langen, R. Structure of membrane-bound alpha-synuclein from site-directed spin labeling and computational refinement. Proc. Natl. Acad. Sci. U. S. A. 105, 19666-19671 (2008).

154. Fusco, G. et al. Direct Observation of the Three Regions in $\alpha$-Synuclein that Determine its Membrane-Bound Behaviour. Nat Commun 1-17 (2014). doi:10.1038/ncomms4827. Direct

155. Kjaer, L., Giehm, L., Heimburg, T. \& Otzen, D. The influence of vesicle size and composition on $\alpha$-synuclein structure and stability. Biophys. J. 96, 2857-2870 (2009).

156. Middleton, E. R. \& Rhoades, E. Effects of curvature and composition on $\alpha$-synuclein binding to lipid vesicles. Biophys. J. 99, 2279-2288 (2010).

157. Jensen, M. B. et al. Membrane curvature sensing by amphipathic helices: A single liposome study using a-synuclein and annexin B12. J. Biol. Chem. 286, 42603-42614 (2011).

158. Binotti, B., Jahn, R. \& Chua, J. J. E. Functions of rab proteins at presynaptic sites. Cells 5, 1-10 (2016).

159. Lesage, $\mathrm{S}$. et al. Loss-of-function mutations in $R A B 39 B$ are associated with typical early-onset Parkinson disease. Neurol. Genet. 1, e9 (2015).

160. Wilson, G. R. et al. Mutations in RAB39B cause X-linked intellectual disability and early-onset parkinson disease with $\alpha$-synuclein pathology. Am. J. Hum. Genet. 95, 729735 (2014).

161. Shi, M., Shi, C. \& Xu, Y. Rab GTPases: The Key Players in the Molecular Pathway of Parkinson's Disease. Front. Cell. Neurosci. 11, 1-8 (2017).

162. Stenmark, H. Rab GTPases as coordinators of vesicle traffic. Nat. Rev. Mol. Cell Biol. 10, 513-525 (2009).

163. Pereira-Leal, J. B. \& Seabra, M. C. The mammalian Rab family of small GTPases: definition of family and subfamily sequence motifs suggests a mechanism for functional specificity in the Ras superfamily 1 1Edited by M. Yaniv. J. Mol. Biol. 301, 1077-1087 (2000).

164. Pereira-Leal, J. B. \& Seabra, M. C. Evolution of the rab family of small GTP-binding proteins. J. Mol. Biol. 313, 889-901 (2001).

165. HUTAGALUNG, A. A. \& NOVICK, P. J. Role of Rab GTPases in Membrane Traffic and Cell Physiology. Physiol Rev 91, 119-149 (2011).

166. Barr, F. \& Lambright, D. G. RAB GEFs and GAPs. Curr. Opin. Cell Biol. 22, 461-470 
(2011).

167. Cherfils, J. \& Zeghouf, M. Regulation of Small GTPases by GEFs, GAPs, and GDIs. Physiol. Rev. 93, 269-309 (2013).

168. Vetter, I. R. The Guanine Nucleotide-Binding Switch in Three Dimensions. Science (80-. ). 294, 1299-1304 (2001).

169. Chutna, O. et al. The small GTPase Rab11 co-localizes with $\alpha$-synuclein in intracellular inclusions and modulates its aggregation, secretion and toxicity. Hum. Mol. Genet. 23, 6732-6745 (2014).

170. Hasegawa, T. et al. The AAA-ATPase VPS4 regulates extracellular secretion and lysosomal targeting of $\alpha$-synuclein. PLoS One 6, (2011).

171. Dalfó, E., Barrachina, M., Rosa, J. L., Ambrosio, S. \& Ferrer, I. Abnormal $\alpha$ synuclein interactions with rab3a and rabphilin in diffuse Lewy body disease. Neurobiol. Dis. 16, 92-97 (2004).

172. Yin, G. et al. $\alpha$-Synuclein interacts with the switch region of Rab8a in a Ser129 phosphorylation-dependent manner. Neurobiol. Dis. 70, 149-161 (2014).

173. Gonçalves, S. A. et al. shRNA-Based Screen Identifies Endocytic Recycling Pathway Components That Act as Genetic Modifiers of Alpha-Synuclein Aggregation, Secretion and Toxicity. PLoS Genet. 12, 1-26 (2016).

174. Chen, R. H. C. et al. a-Synuclein membrane association is regulated by the Rab3a recycling machinery and presynaptic activity. J. Biol. Chem. 288, 7438-7449 (2013).

175. Breda, C. et al. Rab11 modulates $\alpha$-synuclein-mediated defects in synaptic transmission and behaviour. Hum. Mol. Genet. 24, 1077-1091 (2015).

176. Cooper, A. A. et al. a-Synuclein blocks ER-Golgi Traffic and Rab1 Rescues Neuron Loss in Parkinson's Models. Science (80-. ). 313, 324-328 (2007).

177. Outeiro, T. F. \& Lindquist, S. Yeast Cells provide insight into alpha-synuclein biology and pathobiology. Science (80-. ). 302, 1772-1775 (2003).

178. Chua, C. E. L. \& Tang, B. L. Rabs, SNAREs and $\alpha$-synuclein - Membrane trafficking defects in synucleinopathies. Brain Res. Rev. 67, 268-281 (2011).

179. Gitler, A. D. et al. The Parkinson's disease protein -synuclein disrupts cellular Rab homeostasis. Proc. Natl. Acad. Sci. 105, 145-150 (2008).

180. Soper, J. H., Kehm, V., Burd, C. G., Bankaitis, V. A. \& Lee, V. M. Y. Aggregation of $\alpha$-synuclein in S. cerevisiae is associated with defects in endosomal trafficking and phospholipid biosynthesis. J. Mol. Neurosci. 43, 391-405 (2011).

181. Chiu, C.-C. et al. Increased Rab35 expression is a potential biomarker and implicated in the pathogenesis of Parkinson\&amp;rsquo;s disease. Oncotarget 7, 54215-54227 (2016).

182. Sellier, C. et al. Loss of C9ORF72 impairs autophagy and synergizes with polyQ Ataxin-2 to induce motor neuron dysfunction and cell death. EMBO J. 35, 1276-1297 (2016).

183. Granseth, B., Odermatt, B., Royle, S. J. \& Lagnado, L. Clathrin-Mediated 
Endocytosis Is the Dominant Mechanism of Vesicle Retrieval at Hippocampal Synapses. Neuron 51, 773-786 (2006).

184. Alabi, A. A. \& Tsien, R. W. Perspectives on Kiss-and-Run: Role in Exocytosis, Endocytosis, and Neurotransmission. Annu. Rev. Physiol. 75, 393-422 (2013).

185. Doherty, G. \& McMahon, H. Mechanisms of endocytosis. Annu. Rev. Biochem. 78, 857-902 (2009).

186. Staal, R., Mosharov, E. \& Sulzer, D. Dopamine neurons release transmitter via a flickering fusion pore. Nat. Neurosci. 341-346 (2004).

187. Wightman, R. M. \& Haynes, C. L. Synaptic vesicles really do kiss and run. Nat. Neurosci. 7, 321-322 (2004).

188. Royle, S. J. \& Lagnado, L. Clathrin-Mediated Endocytosis at the Synaptic Terminal: Bridging the Gap Between Physiology and Molecules. Traffic 11, 1489-1497 (2010).

189. Vargas, K. J. et al. Synucleins Regulate the Kinetics of Synaptic Vesicle Endocytosis. J. Neurosci. 34, 9364-9376 (2014).

190. Cuervo, A. M. \& Dice, J. F. A Receptor for the Selective Uptake and Degradation of Proteins by Lysosomes. Science (80-. ). 273, 501-503 (1996).

191. Agarraberes, F. a \& Dice, J. F. A molecular chaperone complex at the lysosomal membrane is required for protein translocation. J. Cell Sci. 114, 2491-2499 (2001).

192. Mizushima, N., Ohsumi, Y. \& Yoshimori, T. Autophagosome Formation in Mammalian Cells. Cell Struct. Funct. 27, 421-429 (2002).

193. Hafner Česen, M., Pegan, K., Špes, A. \& Turk, B. Lysosomal pathways to cell death and their therapeutic applications. Exp. Cell Res. 318, 1245-1251 (2012).

194. Mak, S. K., McCormack, A. L., Manning-Bog, A. B., Cuervo, A. M. \& Di Monte, D. A. Lysosomal degradation of a-synuclein in vivo. J. Biol. Chem. 285, 13621-13629 (2010).

195. Cuervo, A. M., Wong, E. S. P. \& Martinez-Vicente, M. Protein degradation, aggregation, and misfolding. Mov. Disord. 25, (2010).

196. Winslow, A. R. et al. $\alpha$-Synuclein impairs macroautophagy: Implications for Parkinson's disease. J. Cell Biol. 190, 1023-1037 (2010).

197. Tanik, S. A., Schultheiss, C. E., Volpicelli-Daley, L. A., Brunden, K. R. \& Lee, V. M. Y. Lewy body-like a-synuclein aggregates resist degradation and impair macroautophagy. J. Biol. Chem. 288, 15194-15210 (2013).

198. Yan, J. Q. et al. Overexpression of Human E46K Mutant $\alpha$-Synuclein Impairs Macroautophagy via Inactivation of JNK1-Bcl-2 Pathway. Mol. Neurobiol. 50, 685701 (2014).

199. Alvarez-Erviti, L. et al. Lysosomal dysfunction increases exosome-mediated alphasynuclein release and transmission. Neurobiol. Dis. 42, 360-367 (2011).

200. Lee, H. J. et al. Autophagic failure promotes the exocytosis and intercellular transfer of $\alpha$-synuclein. Exp. Mol. Med. 45, e22-9 (2013). 
201. Falkenburger, B. H., Saridaki, T. \& Dinter, E. Cellular models for Parkinson's disease. J. Neurochem. 121-130 (2016). doi:10.1111/jnc.13618

202. Lázaro, D. F., Pavlou, M. A. S. \& Outeiro, T. F. Cellular models as tools for the study of the role of alpha-synuclein in Parkinson's disease. Exp. Neurol. 298, 162-171 (2017).

203. Roostaee, A., Beaudoin, S., Staskevicius, A. \& Roucou, X. Aggregation and neurotoxicity of recombinant $\alpha$-synuclein aggregates initiated by dimerization. Mol. Neurodegener. 8, 5 (2013).

204. Krishnan, S. et al. Oxidative dimer formation is the critical rate-limiting step for Parkinson's disease alpha-synuclein fibrillogenesis. Biochemistry 42, 829-837 (2003).

205. Kerppola, T. Design and implementation of bimolecular fluorescence complementation (BiFC) assays for the visualization of protein interactions in living cells. Nat. Protoc. 1, 1278-1286 (2006).

206. Gonçalves, S. A., Matos, J. E. \& Outeiro, T. F. Zooming into protein oligomerization in neurodegeneration using BiFC. Trends Biochem. Sci. 35, 643-651 (2010).

207. Outeiro, T. F. et al. Formation of toxic oligomeric $\alpha$-synuclein species in living cells. PLoS One 3, 1-9 (2008).

208. McLean, P. J., Kawamata, H. \& Hyman, B. T. $\alpha$-Synuclein-enhanced green fluorescent protein fusion proteins form proteasome sensitive inclusions in primary neurons. Neuroscience 104, 901-912 (2001).

209. Swinnen, E. et al. Aggresome formation and segregation of inclusions influence toxicity of $\alpha$-synuclein and synphilin-1 in yeast. Biochem. Soc. Trans. 39, 1476-1481 (2011).

210. Büttner, S. et al. Synphilin-1 enhances a-synuclein aggregation in yeast and contributes to cellular stress and cell death in a sir2-dependent manner. PLoS One 5, (2010).

211. Lücking, C. B. \& Brice, A. Review Alpha-synuclein and Parkinson's disease. C. Cell. Mol. Life Sci 57, 1894-1908 (2000).

212. Engelender, S. et al. Synphilin-1 associates with alpha-synuclein and promotes the formation of cytosolic inclusions. Nat.Genet. 22, 110-114 (1999).

213. Klucken, J. et al. Alpha-synuclein aggregation involves a bafilomycin A 1-sensitive autophagy pathway. Autophagy 8, 754-766 (2012).

214. Poehler, A. M. et al. Autophagy modulates SNCA/ $\alpha$-synuclein release, thereby generating a hostile microenvironment. Autophagy 10, 2171-2192 (2014).

215. Johnson, M., Geeves, M. A. \& Mulvihill, D. P. Production of amino-terminally acetylated recombinant proteins in E . coli. Methods Mol Biol 1-7 (2015). doi:10.1007/978-1-62703-305-3

216. Elia, G. Biotinylation reagents for the study of cell surface proteins. Proteomics $\mathbf{8}$, 4012-4024 (2008).

217. Scheurer, S. B. et al. Identification and relative quantification of membrane proteins by surface biotinylation and two-dimensional peptide mapping. Proteomics 5, 2718$2728(2005)$. 
218. Schanda, P., Kupĉe, E. \& Brutscher, B. SOFAST-HMQC experiments for recording two-dimensional deteronuclear correlation spectra of proteins within a few seconds. $J$. Biomol. NMR 33, 199-211 (2005).

219. Miotto, M. C. et al. Copper Binding to the N-Terminally Acetylated, Naturally Occurring Form of Alpha-Synuclein Induces Local Helical Folding. J. Am. Chem. Soc. 137, 6444-6447 (2015).

220. Lamberto, G. R. et al. Structural and mechanistic basis behind the inhibitory interaction of PcTS on alpha-synuclein amyloid fibril formation. Proc. Natl. Acad. Sci. U. S. A. 106, 21057-21062 (2009).

221. Farrow, N. A., Zhang, O., Forman-Kay, J. D. \& Kay, L. E. Comparison of the backbone dynamics of a folded and an unfolded SH3 domain existing in equilibrium in aqueous buffer. Biochemistry 34, 868-878 (1995).

222. Binolfi, A., Fernández, C. O., Sica, M. P., Delfino, J. M. \& Santos, J. Recognition between a short unstructured peptide and a partially folded fragment leads to the thioredoxin fold sharing native-like dynamics. Proteins Struct. Funct. Bioinforma. 80, 1448-1464 (2012).

223. Serrano, L. Comparison between the phi distribution of the amino acids in the protein database and NMR data indicates that amino acids have various phi propensities in the random coil conformation. J. Mol. Biol. 254, 322-333 (1995).

224. Villar-Piqué, A. et al. Environmental and genetic factors support the dissociation between $\alpha$-synuclein aggregation and toxicity. Proc. Natl. Acad. Sci. 201606791 (2016). doi:10.1073/pnas. 1606791113

225. Konstantinopoulos, P. A., Karamouzis, M. V. \& Papavassiliou, A. G. Posttranslational modifications and regulation of the RAS superfamily of GTPases as anticancer targets. Nat. Rev. Drug Discov. 6, 541-555 (2007).

226. D'Souza, R. S. et al. Rab4 orchestrates a small GTPase cascade for recruitment of adaptor proteins to early endosomes. Curr. Biol. 24, 1187-1198 (2014).

227. Burré, J., Sharma, M. \& Südhof, T. C. Systematic mutagenesis of aSyn reveals distinct sequence requirements for physiological and pathological activities. $J$. Neurochem. 1522715242 (2012). doi:10.1016/j.jacc.2007.01.076. White

228. Burré, J., Sharma, M. \& Südhof, T. C. Definition of a Molecular Pathway Mediating a-Synuclein. J. Neurosci. 35, 5221-5232 (2015).

229. Fonseca-Ornelas, L. et al. Small molecule-mediated stabilization of vesicle-associated helical $\alpha$-synuclein inhibits pathogenic misfolding and aggregation. Nat. Commun. 5, 5857 (2014).

230. Lee, J. H., Ying, J. \& Bax, A. Nuclear Magnetic Resonance Observation of aSynuclein Membrane Interaction by Monitoring the Acetylation Reactivity of Its Lysine Side Chains. Biochemistry 55, 4949-4959 (2016).

231. Smith, C. M., Haucke, V., McCluskey, A., Robinson, P. J. \& Chircop, M. Inhibition of clathrin by pitstop 2 activates the spindle assembly checkpoint and induces cell death in dividing HeLa cancer cells. Mol. Cancer 12, 1-15 (2013).

232. Soohoo, A. L. \& Puthenveedu, M. a. Divergent modes for cargo-mediated control of clathrin-coated pit dynamics. Mol. Biol. Cell 24, 1725-34, S1-12 (2013). 
233. Mccluskey, A. et al. Building a better dynasore: The dyngo compounds potently inhibit dynamin and endocytosis. Traffic 14, 1272-1289 (2013).

234. Lázaro, D. F. et al. Systematic Comparison of the Effects of Alpha-synuclein Mutations on Its Oligomerization and Aggregation. PLoS Genet. 10, (2014).

235. Ormo, M. et al. Crystal Structure of the Aequorea victoria Green Fluorescent Protein. Science (80-. ). 273, 1392-1395 (2016).

236. Minch, M. J. Orientational dependence of vicinal proton-proton NMR coupling constants: The Karplus relationship. Concepts Magn. Reson. 6, 41-56 (1994).

237. Cho, M. K. et al. Structural characterization of a-synuclein in an aggregation prone state. Protein Sci. 18, 1840-1846 (2009).

238. Valiente-Gabioud, A. A. et al. Phthalocyanines as Molecular Scaffolds to Block Disease-Associated Protein Aggregation. Acc. Chem. Res. 49, 801-808 (2016).

239. Pacheco, C., Aguayo, L. G. \& Opazo, C. An extracellular mechanism that can explain the neur effects of $\alpha$-synuclein aggregates in the brain. Front. Physiol. 3 JUL, $1-10(2012)$.

240. Ahn, K. J., Paik, S. R., Chung, K. C. \& Kim, J. Amino acid sequence motifs and mechanistic features of the membrane translocation of a-synuclein. J. Neurochem. 97, 265-279 (2006).

241. Tang, B. L. Rabs, Membrane Dynamics, and Parkinson's Disease. J. Cell. Physiol. 232, 1626-1633 (2017).

242. Sönnichsen, B., De Renzis, S., Nielsen, E., Rietdorf, J. \& Zerial, M. Distinct membrane domains on endosomes in the recycling pathway visualized by multicolor imaging of Rab4, Rab5, and Rab11. J. Cell Biol. 149, 901-913 (2000).

243. Kisos, H., Ben-Gedalya, T. \& Sharon, R. The clathrin-dependent localization of dopamine transporter to surface membranes is affected by $\alpha$-Synuclein. J. Mol. Neurosci. 52, 167-176 (2014).

244. Cheng, F. et al. $\alpha$-Synuclein promotes clathrin-mediated NMDA receptor endocytosis and attenuates NMDA-induced dopaminergic cell death. J. Neurochem. 119, 815-825 (2011).

245. Ben, G. T. et al. Alpha-synuclein and polyunsaturated fatty acids promote clathrin mediated endocytosis and synaptic vesicle recycling. Traffic 10, 218-234 (2009).

246. Busch, D. J. et al. Acute increase of $\alpha$-synuclein inhibits synaptic vesicle recycling evoked during intense stimulation. Mol. Biol. Cell 25, 3926-41 (2014).

247. Michel, C. H. et al. Extracellular monomeric tau protein is sufficient to initiate the spread of tau protein pathology. J. Biol. Chem. 289, 956-967 (2014).

248. Casey, J. R., Grinstein, S. \& Orlowski, J. Sensors and regulators of intracellular pH. Nat. Rev. Mol. Cell Biol. 11, 50-61 (2010).

249. Lindersson, E. et al. p25a Stimulates a-Synuclein Aggregation and Is Co-localized with Aggregated a-Synuclein in a-Synucleinopathies *. J. Biol. Chem. 280, 5703-5715 (2005). 
250. Dias, V., Junn, E. \& Mouradian, M. M. The Role of Oxidative Stress in Parkinson's Disease. J Park. Dis 3, 461-491 (2014).

251. Valiente-Gabioud, A. A. et al. Structural basis behind the interaction of $\mathrm{Zn} 2+$ with the protein $\alpha$-synuclein and the A $\beta$ peptide: A comparative analysis. J. Inorg. Biochem. 117, 334-341 (2012).

252. Wolozin, B. \& Golts, N. Iron and Parkinson 's Disease. Neurosci. 60153, 22-32 (2002).

253. Opazo, F., Krenz, A., Heermann, S., Schulz, J. B. \& Falkenburger, B. H. Accumulation and clearance of $\alpha$-synuclein aggregates demonstrated by time-lapse imaging. J. Neurochem. 106, 529-540 (2008).

254. Gomes-Trolin, C., Nygren, I., Aquilonius, S.-M. \& Askmark, H. Increased Red Blood Cell Polyamines in ALS and Parkinson's Disease. Exp. Neurol. 177, 515-520 (2002).

255. Lu, Y., Prudent, M., Fauvet, B., Lashuel, H. A. \& Girault, H. H. Phosphorylation of a-synuclein at Y125 and S129 alters its metal binding properties: Implications for understanding the role of a-synuclein in the pathogenesis of Parkinson's disease and related disorders. ACS Chem. Neurosci. 2, 667-675 (2011).

256. Li, W. et al. Aggregation promoting C-terminal truncation of alpha-synuclein is a normal cellular process and is enhanced by the familial Parkinson's disease-linked mutations. Proc. Natl. Acad. Sci. U. S. A. 102, 2162-7 (2005).

257. Beyer, K. \& Ariza, A. Alpha-Synuclein Posttranslational Modification and Alternative Splicing as a Trigger for Neurodegeneration. Mol. Neurobiol. 1-16 (2012). doi:10.1007/s12035-012-8330-5 


\section{ACKNOWLEDGEMENTS}

To begin this journey, and even more, to conclude it, was not easy.

It was a period full of frustration, full of disappointment, and lately, full of awareness. However, people say that the best things come from the uncomfortable, painful moments and I am confident none of this happened in vain.

My first thanks go to Prof. Dr. Tiago Outeiro, for giving me the opportunity to conduct my doctoral studies at the Department of Experimental Neurodegeneration in Göttingen, providing me with the necessary equipment, time and space to develop my research and my ideas.

On the same way, I need to thank my co-supervisor, Prof. Dr. Claudio Fernández, for allowing me to perform part of my experiment in his lab, at the MPLbioR, UNRSociedad Max Planck in Rosario. The period I spent there was one of the happiest moment of my $\mathrm{PhD}$, turning in an incredibly amusing experience both from a professional and a personal side.

I also want to thank the member of my thesis committee, Prof. Dr. Markus Zweckstetter and Prof. Gerhard Braus, for the cooperation and the helpful suggestions received during the meetings. Thanks as well to the further members of my Examination Board, Dr. Ira Milosevic, Dr. Mauricio Menacho Marquez and Prof. Reinhard Jahn, for taking the time to be present at my $\mathrm{PhD}$ Defense.

A huge, grateful thank you goes to my two scientific mentors, one for each hemisphere of the Earth: Dr. Andrés Binolfi and Dr. Ira Milosevic.

I will be eternally grateful to Andrés for showing me the kind of person I want to be: a brilliant - extremely brilliant - scientist that look at the world with a critical eye, a childlike spirit but yet with extreme humbleness and with the conscious that we everyone - have still, and always, a lot to learn.

Thank you Andrés, thank you for supervising me so carefully and thank you for listening to all my ideas, even the silliest, without ever discouraging or crushing me.

To Ira goes my deepest gratitude for showing me how beautiful is to preserve and to promote such a genuine passion for Science. For showing me that is possible to be a woman, to have a family, to manage a lab and to do excellent research, altogether.

Thank you Ira, for being always so enthusiastic about our collaboration, for believing in me since the beginning and for motivating me. Thank you for your time, for all the precious advices, for the professional and personal suggestions, for all the help. 
I should also thank my colleagues and former colleagues: Joana, Francisca, Tomás and his brilliant ideas, Isabel, Mariana, the other Mariana, Maria, Vaishali, Omar and Dani, my queridisima Anka and Laetitia The Warrior, for all the moment spent together, for the help, for the support, for the lunch together and all the talk.

To Elly goes a special thank, from the bottom of my heart, for being the strongest, and for all the wine and the extremely pleasant dinners together.

It was wonderful Elly, and I am honoured to be your friend! (Oh my god! I have a German Friend! Can you believe it???)

A special mention goes to Shamina.

Grazie more for saving me from mental illness in lovely, warm and friendly Göttingen. Without you, I wouldn't probably manage it. Ti voglio bene Shamina!

Thank as well to Frau Karboni, to Chiappetta, to Judith and Moritz, to make some of my days - and the Department Party - funnier and lighter.

A huge thank goes to all the people I met in Argentina, where I spent one of the greatest year of my life, where I could feel at home, where I was always smiling, where everything was easy even if nothing ever - EVER - works, but well, Who cares?

It is sunny outside, tomate un mate, hacemo un asado, Che!

Thanks to Mauge, Vero, Pablito, Charliero, Franco, Timo, Flor, Antonio, Gonzalito (sos el orgullo del pais Gonzalito, pero vamos en Cambridge, que increible!!!) y Marcolino, obviamente!

Thanks to Tomé, for being the nicest person, the nicest friend, the nicest Apoyo Tecnico in the entire world! You deserve the best, Tomé, and I am really lucky to have meet you, and extremely grateful to be your friend.

To Ariel, or better, to Dr. Valiente, goes all my sympathy, my affection and my admiration, before than all my thanks, for being such a good and funny - incredibly funny - friend, such a precise, methodical, impeccable scientist and collaborator, for his humour above the lines, for the trips, the time and the philosophy about work and politics done together. Muchisimas gracias Ariel, por ser exactamente como eres!

Thanks to my old and less old friends: La Giulia, the crazy part of me, my beautiful, wonderful Sofia, the person everyone should know and fall in love with, La Prunety, that follow me even in the other side of the Ocean (Non te ne andare Prunetyyyyyy, 
non te ne andareeeeeeeeee!), and Sarah, my crucca but not-at-all crucca friend, and of course, Shamina again.

Thanks to all of you, you are literally the best part of every place, every experience, and every fundamental step of my life.

Last but not least, Thanks to my Family.

Young Rita would never though Old Rita would become so sentimental, so attached to the family, so worried and concerned and homesick for Mummy and Papino.

But well, la vecchiaia é una brutta bestia, so...

A simple "thank you" will never be enough, for all the efforts, the time, the money, the concerns and everything else you spent on me. For me.

What to say? I just hope you could feel proud even $1 / 10$ of how much I feel proud, lucky and grateful to have you.

As a special part of my family, thanks to you Mon Amour.

You are definitely the best thing coming from my $\mathrm{PhD}$, from these last 4 years.

And - as you know - I couldn't have asked for more.

I couldn't even have imagined more.

So, thank you for being my special Unicorn.

Love you chatinho! 


\section{Caterina Masaracchia}

\section{Personal InFormation}

Current address: Heidelberg, Baden-Württemberg, Germany.

Date of Birth:

Citizenship: 08 April 1987

Languages: Italian

Italian

(mother tongue)

English and Spanish (full professional proficiency)

French

(intermediate proficiency)

German

(elementary proficiency)

\section{EDUCATION}

International PhD on Biosciences and Molecular Biomedicine

2014- current

Georg-August Universität, Göttingen - Germany - and

MPLbioR, UNR-Sociedad Max Planck - Rosario, Santa Fe - Argentina

Master Degree in Molecular and Cellular Biology

Alma Mater Studiorum. University of Bologna - Bologna, Italy

110/110 cum laude (with honors)

Bachelor Degree in Biological Sciences

$2006-2010$

Alma Mater Studiorum. University of Bologna - Bologna, Italy

\section{APPOINTMENTS}

\section{CUAA-DAHZ International PhD on Biosciences and Molecular Biomedicine and PhD in Biology - GAUSS Program \\ 2014- current}

Dissertation: "Analysis of the mechanisms of interaction of alpha-synuclein and membranes in cellular models of Parkinson's Disease"

Georg-August University of Gottingen - Department of Experimental

Neurodegeneration - Georg-August Universität - Göttingen, Germany

Supervisor : Prof. Dr. Tiago Fleming Outeiro

Institute for the Drug discovery of Rosario (IIDEFAR, CONICET-UNR) and (MPLbioR, UNR-Sociedad Max Planck) - Rosario, Santa Fe - Argentina Supervisors: Prof. Dr. Claudio O. Fernández and Dr. Andrés Binolfi 
"The Ageing Brain"

U4 Network - Göttingen, Germany

John Van Geest Centre for Brain Repair Spring School 2014

"The Spreading Pathology of Neurodegenerative disorders"

Department of Clinical Neuroscience, University of Cambridge - Cambridge, UK

\section{Visiting Researcher}

Apr. 2013 - Feb. 2014

UNI, ULB Neuroscience Institute

Laboratory of General Histology, Neuroanathomy and Neuropathology

ULB, Univeristè Libre de Bruxelles - Bruxelles, Belgium

Supervisor: Prof. Jean-Pierre Brion

\section{Master Degree's Internship}

Feb. 2012 - Nov. 2012

Molecular Pathology Laboratory,

Department of Experimental, Diagnostic and Specialty Medicine - Unity of

Hematopathology - Bologna University Medical School - Bologna, Italy

Supervisor: Prof. Dr. Stefano Pileri

\section{Bachelor Degree's Internship}

Nov 2009 - Feb 2010

Department of Pharmacy and Biotechnology,

Alma Mater Studiorum, University of Bologna - Bologna, Italy

Supervisor: Prof. Paolo Bernardo Trost

\section{PROFESSIONAL SKILLS}

Cellular Biology: cell cultures; vitality and proliferation essays (CellTiter Glo, Alamar Blue, MTT assay); transfections (calcium phosphate, lipofectamine); immunocytochemistry, immunoblotting and electrophoresis. Imaging and live imaging (Fluorescence, Confocal, Spin Disc Microscopy)

Molecular Biology: plasmid cloning, bacterial transformation, extraction and purification of nucleic acid from FFPE tissues, human blood, cryopreserved samples. Extraction and purification of recombinant proteins, High-Pressure Liquid Chromatography (HPLC), aggregation assay.

Structural Biology: Nuclear Magnetic Resonance (NMR)

Computer Skills: Office, ImageJ, Graph Pad, CorelDraw, CCPN, TopSpin.

Teaching: Supervision of Master students during lab rotations (Saskia Kohen, Marilena Hnida); Supervision of visiting researcher in practical work - (Maria Eugenia Chesta and Mauricio Menacho) 


\section{Presentations AND PANELS}

- Biophisical characterization of an aggregation-prone variant of alpha-synuclein as a model of synucleinopathies - AD/PD 2017:13th International Conference on Alzheimer's Disease and Parkinson's Disease - Vienna, Austria (poster presentation)

- The interaction between $a$-synuclein, membranes and trafficking machinery: insights into the mechanisms of spreading; C2 Retreat, April 2015 - Göttingen, Germany (poster presentation)

- Internalization and behavior of monomeric, oligomeric and filamentous aSyn in cultured cells - U4 Graduate School 2014 - Göttingen, Germany (poster presentation)

\section{Publications}

- High-Molecular-Weight Paired Helical Filaments from Alzheimer Brain Induces Seeding of Wild-Type Mouse Tau into an Argyrophilic 4R Tau Pathology in Vivo

Audouard E, Houben S, Masaracchia C, Yilmaz Z, Suain V, Authelet M, De Decker R, Buée L, Boom A, Leroy K, Ando K, Brion JP. Am J Pathol. 2016 Oct;186(10):2709-22. doi: 10.1016/j.ajpath.2016.06.008. PMID: 27497324

- Environmental and genetic factors support the dissociation between $\alpha$-synuclein aggregation and toxicity

Villar-Piqué A, Lopes da Fonseca T, Sant'Anna R, Szegö ÉM, Fonseca-Ornelas L, Pinho R, Carija A, Gerhardt E, Masaracchia C, Abad Gonzalez E, Rossetti G, Carloni P, Fernández CO, Foguel D, Milosevic I, Zweckstetter M, Ventura S, Outeiro TF. Proc Natl Acad Sci U S A. 2016 Oct 5. pii: 201606791.

PMID: 27708160

- Characterization of an aggregation-prone variant of alpha-synuclein as a model for synucleinopathies

Masaracchia C, Valiente-Gabioud AA, Peralta P, Favretto F, Strohäker T, Lázaro FD, Zweckstetter M, Binolfi A, Fernandez CO, Outeiro TF

- Submitted to Biochemistry - 
